\title{
Young stellar populations in type II quasars: timing the onset of star formation and nuclear activity
}

\author{
P. S. Bessiere, ${ }^{1,2 \star}$ C. N. Tadhunter,${ }^{2}$ C. Ramos Almeida,,${ }^{3,4}$ M. Villar Martín ${ }^{5}$ \\ and A. Cabrera-Lavers ${ }^{3,4,6}$ \\ ${ }^{1}$ Instituto de Astrofísica, Facultad de Física, Pontificia Universidad Católica de Chile, Casilla 306, Santiago 22, Chile \\ ${ }^{2}$ Department of Physics and Astronomy, University of Sheffield, Sheffield S3 7RH, UK \\ ${ }^{3}$ Instituto de Astrofísica de Canarias (IAC), C/Vía Láctea, s/n, E-38205 La Laguna, Tenerife, Spain \\ ${ }^{4}$ Departamento de Astrofísica, Universidad de La Laguna, E-38205 La Laguna, Tenerife, Spain \\ ${ }^{5}$ Centro de Astrobiología (INTA-CSIC), Carretera de Ajalvier, km 4, E-28850 Torrejón de Ardoz, Madrid, Spain \\ ${ }^{6}$ Gran Telescopio CANARIAS, Cuesta de San José, s/n, E-38712 Breña Baja, La Palma, Spain
}

Accepted 2016 December 5. Received 2016 November 25; in original form 2016 May 11

\begin{abstract}
Despite the emerging morphological evidence that luminous quasar-like active galactic nuclei (AGN) are triggered in galaxy mergers, the natures of the triggering mergers and the order of events in the triggering sequence remain uncertain. In this work, we present a detailed study of the stellar populations of the host galaxies of 21 type II quasars, with the aim of understanding the sequence of events between the onset of the merger, the triggering of the associated starburst and the initiation of the quasar activity. To this end, we model highquality, wide spectral coverage, intermediate-resolution optical spectra of the type II quasars. We find that of the 21 objects, the higher order Balmer absorption lines, characteristic of young stellar populations (YSPs), are directly detected in $\sim 62$ per cent of the sample. We also fit these spectra using a number of combinations of stellar and/or power-law components, representative of viable formation histories, as well as including the possibility of scattered AGN light. We find that $\sim 90$ per cent of the type II quasar host galaxies require the inclusion of a YSP to adequately model their spectra, whilst 71 per cent of the sample require the inclusion of a YSP with age $<100 \mathrm{Myr}$. Since the ages of the YSP in most type II quasar host galaxies are comparable with the expected lifetimes of the AGN activity, these results provide strong evidence that the quasars are triggered close to the peaks of the merger-induced starbursts.
\end{abstract}

Key words: galaxies: active-galaxies: evolution-galaxies: interactions-galaxies: stellar content.

\section{INTRODUCTION}

In recent years the role of active galactic nuclei (AGN) in influencing the evolution of massive galaxies has come to the fore. This is because strong correlations have been found between the masses of the supermassive black holes (SMBH), which lay at the heart of nearly all massive galaxies (Magorrian et al. 1998), and the galaxy bulges they inhabit (Ferrarese \& Merritt 2000; Gebhardt et al. 2000). In spite of their apparent significance in shaping local correlations, we still do not have a clear understanding of the processes that trigger this activity.

One credible scenario is that AGNs, and in particular highluminosity (quasar-like) AGNs, are triggered by mergers. The attraction of this mechanism is that it helps to solve one of the major problems associated with triggering luminous AGN, namely, how do the gas in a galaxy sheds over 99 per cent of its angular momentum so that it can move from galaxy-wide scales down to the subparsec scale where it can accrete on to the central SMBH (e.g. Heckman et al. 1986; Hopkins et al. 2006). A number of studies investigating the importance of mergers have shown that they can play a significant role (Bennert et al. 2008; Ramos Almeida et al. 2011; Bessiere et al. 2012); indeed, Treister et al. (2012) find a steep increase in merger rate with increasing AGN luminosity. It is also interesting to note that both Ramos Almeida et al. (2012) and Bessiere et al. (2012) find that the surface brightnesses of the tidal features associated with the mergers are up to two magnitudes brighter than those associated with a matched sample of quiescent galaxies. As discussed in Bessiere et al. (2012), this could indicate that the types of mergers being witnessed in luminous AGNs and their matched quiescent control samples are fundamentally different (i.e. wet versus dry/major versus minor). 
Tidal disruption of the host galaxies will not be the only consequence of mergers. Simulations suggest that, at the point of coalescence, not only is there a peak in the accretion rate on to the $\mathrm{SMBH}$, but the gas driven into the nuclear regions also triggers a burst of star formation (Springel, Di Matteo \& Hernquist 2005; Hopkins et al. 2006). If this is the case, using stellar synthesis modelling techniques to date the starburst (e.g. Canalizo \& Stockton 2000, 2001; Tadhunter et al. 2002; Holt et al. 2007), we can determine a fiducial time for the peak of the galaxy merger. In this way, it is possible to gain insights into the order of events and timing of the quasar activity in relation to the merger - key information for understanding the co-evolution of quasars and their host galaxies.

Stellar population synthesis modelling can also be used to test evolutionary scenarios for the triggering of quasars in mergers. For example, Sanders et al. (1988) propose a scenario in which mergers between gas-rich galaxies trigger prodigious star formation, which will manifest itself as an ultraluminous infrared galaxy (ULIRG). As the merger progresses and the two nuclei coalesce, the quasar activity begins to dominate the bolometric luminosity, eventually driving out the envelope of dust and gas, and allowing the quasar to become clearly detectable in the optical (Springel et al. 2005; Hopkins et al. 2006). The progression of such a sequence means that the burst of star formation will occur while the quasar is shrouded by its natal cocoon. Therefore, by the time the quasar emerges, we would expect to observe that the starburst stellar population has already aged. Both Ramos Almeida et al. (2011) and Bessiere et al. (2012) find this scenario to be somewhat oversimplistic as both studies show that the AGN activity can be triggered before, during, or well after the coalescence of the SMBH. However, such morphological studies do not reveal any fine detail in terms of the nature of the merger and the relative timing of the starburst and AGN activity.

Previous investigations into the stellar populations of AGN host galaxies have produced mixed results. While some find that the triggering of the AGN activity and the starburst are quasi-simultaneous (Heckman et al. 1997; Canalizo \& Stockton 2000; Brotherton et al. 2002; Davies et al. 2007; Wills et al. 2008; Tadhunter et al. 2011; Bessiere et al. 2014) and the ages of the young stellar populations (YSPs) are less than the expected maximum lifetime of an AGN of 100 Myr (Martini \& Weinberg 2001), other works find a clear delay in the triggering of the AGN relative to the starburst, with the ages of the stellar populations ranging from a few 100 Myr to a few Gyr (Tadhunter et al. 2005; Holt et al. 2007; Wills et al. 2008; Wild, Heckman \& Charlot 2010; Canalizo \& Stockton 2013).

In order to try to understand the root of these differences, we have carried out a campaign of long-slit optical spectroscopic observations of a 95 per cent complete sample of type II quasars selected from the catalogue of candidate type II objects presented by Zakamska et al. (2003) with [O III] $\lambda 5007$ emission line luminosities $\left(L_{[\mathrm{O} \text { III }}\right) L_{[\mathrm{O} \text { III }]}>10^{8.5} \mathrm{~L} \odot$. We exclusively consider type II quasars in this work because they are ideal objects for making detailed studies of the host galaxies of luminous AGN. In type II quasars, the central engine, which would otherwise overwhelm the host galaxy light, is obscured from view by intervening material. Therefore, there is no need to attempt to model and subtract the central point source before embarking on a detailed study of the underlying host galaxy.

A full morphological analysis of this sample has already been presented in Bessiere et al. (2012), and a detailed analysis of the stellar populations of one of the type II quasars (J0025-10) has also been presented in Bessiere et al. (2014). J0025-10 provides strong evidence for young YSP ages and the simultaneous triggering of the quasar and the merger-induced starburst, but is this true of type II quasar host galaxies in general? Here, we present the analysis of the remaining 18 objects from the original sample for which we have spectra, as well as two further type II quasars for which high-quality long-slit spectra have subsequently been obtained. We have used the spectra to determine the ages and reddenings of the young to intermediate-age stellar populations (YSPs/ISPs; $0.001 \leqq$ $\left.t_{\mathrm{YSP}}(\mathrm{Gyr}) \leqq 5\right)$ that may be present in the host galaxies of these type II quasars.

Throughout this paper, we assume a cosmology with $H_{0}=$ $70 \mathrm{kms}^{-1} \mathrm{Mpc}^{-1}, \Omega_{\mathrm{M}}=0.3$ and $\Omega_{\Lambda}=0.7$.

\section{SAMPLE, OBSERVATIONS AND DATA REDUCTION}

The original sample of 20 type II quasars was derived from the catalogue of candidate objects established by Zakamska et al. (2003), who selected galaxies from the Sloan Digital Sky Survey (SDSS; York et al. 2000) with high-ionization, narrow emission lines, but no indication of broad permitted lines. To characterize the nature of the ionizing source, they used diagnostic emission line ratios to demonstrate the energetic dominance of the AGN. Zakamska et al. (2003) provide full information on the criteria used to select type II objects based on emission line ratios.

Because the AGN is itself obscured in type II objects, the luminosity of the $[\mathrm{O} \mathrm{III}] \lambda 5007$ emission line is used as a proxy for its bolometric luminosity (e.g. Heckman et al. 2005; LaMassa et al. 2009; Dicken et al. 2014). It is assumed that a type II object will have the same intrinsic AGN luminosity for a given $L_{[\mathrm{O} \text { II] }}$ as a type I object. Zakamska et al. (2003) make their cut between objects that are considered to be quasars, and those with Seyfert-like luminosities at $L_{\text {[O III }}>10^{8.5} \mathrm{~L}_{\odot}$, which is roughly equivalent to an AGN absolute magnitude $M_{B}<-23$ mag.

The sample presented in this work comprises a 95 per cent complete sample of 19 of the 20 objects from Zakamska et al. (2003) with RAs in the range $23<\mathrm{RA}<10 \mathrm{~h}, \delta<+20^{\circ}$, redshifts in the range $0.3<z<0.41$ and [O III] emission line luminosities $L_{[\mathrm{O} \text { III }]}>10^{8.5} \mathrm{~L}_{\odot}$ (see Table 1$)$. This includes results for J0025-10, which have already been presented in Bessiere et al. (2014). Unfortunately, for 1 of the 20 objects in the full sample $(\mathrm{J} 0159+14)$, we were unable to obtain spectroscopic data of sufficient quality attempt modelling of the stellar populations. Given the high level of completeness of our sample, we are not biased towards a particular luminosity or optical morphology in terms of host galaxy properties.

When determining our selection criteria, the $L_{[\mathrm{O} \text { III] }}$ limit was chosen to ensure that the objects do indeed contain AGN with quasar-like bolometric luminosities. However, subsequent to the publication of candidate objects by Zakamska et al. (2003), the SDSS spectrophotometric calibration pipeline has been updated and improved (Abazajian et al. 2004; Adelman-McCarthy et al. 2007). Using the same sample selection criteria and employing the same line fitting technique, Reyes et al. (2008) have since updated the emission line luminosities presented by Zakamska et al. (2003) using data calibrated with this improved pipeline. These updated luminosities suggest that six objects from the original sample selection fall marginally below our $L_{[\mathrm{O} \text { III] }}$ cut. However, because the original sample selection was based on Zakamska et al. (2003), we choose to retain the full sample of 19 objects, although the updated $L_{\text {[O III] }}$ values are included in Table 1 (bracketed values) as a matter of interest. It is also worth noting that were a luminosity cut of 
Table 1. Full classification of the sample objects. Columns 1 and 2 give the SDSS identifier and abbreviated identifier that will be used throughout this work. Columns 3 and 4 list the spectroscopic redshift and cosmology corrected scale taken from the NED data base. Column 5 corresponds to the value of $\log \left(L_{[\mathrm{O} I I]} / \mathrm{L}_{\odot}\right)$ taken from Zakamska et al. (2003) upon which the original sample selection was based, while the values in brackets are updated values taken from Reyes et al. (2008).

\begin{tabular}{lcccc}
\hline Name & $\begin{array}{c}\text { Abbreviated } \\
\text { name }\end{array}$ & $z$ & $\begin{array}{c}\text { Scale } \\
\left(\mathrm{kpc} \mathrm{arcsec}^{-1}\right)\end{array}$ & $\begin{array}{c}\log \\
\left(L_{[\mathrm{O} I \mathrm{II}]} / \mathrm{L}_{\odot}\right)\end{array}$ \\
\hline $\mathrm{J} 235818-000919$ & $\mathrm{~J} 2358-00$ & 0.402 & 5.39 & $9.32(9.29)$ \\
$\mathrm{J} 002531-104022$ & $\mathrm{~J} 0025-10$ & 0.303 & 4.48 & $8.73(8.65)$ \\
$\mathrm{J} 011429+000037$ & $\mathrm{~J} 0114+00$ & 0.389 & 5.28 & $8.66(8.46)$ \\
$\mathrm{J} 012341+004435$ & $\mathrm{~J} 0123+00$ & 0.399 & 5.36 & $9.13(9.14)$ \\
$\mathrm{J} 014237+144117$ & $\mathrm{~J} 0142+14$ & 0.389 & 5.28 & $8.76(8.87)$ \\
$\mathrm{J} 021757-011324$ & $\mathrm{~J} 0217-01$ & 0.375 & 5.16 & $8.55(8.37)$ \\
$\mathrm{J} 021758-001302$ & $\mathrm{~J} 0217-00$ & 0.344 & 4.88 & $8.75(8.81)$ \\
$\mathrm{J} 021834-004610$ & $\mathrm{~J} 0218-00$ & 0.372 & 5.13 & $8.85(8.62)$ \\
$\mathrm{J} 022701+010712$ & $\mathrm{~J} 0227+01$ & 0.363 & 5.05 & $8.90(8.70)$ \\
$\mathrm{J} 023411-074538$ & $\mathrm{~J} 0234-07$ & 0.310 & 4.55 & $8.77(8.78)$ \\
$\mathrm{J} 024946+001003$ & $\mathrm{~J} 0249+00$ & 0.408 & 5.44 & $8.63(8.75)$ \\
$\mathrm{J} 032029+003153$ & $\mathrm{~J} 0320+00$ & 0.384 & 5.23 & $8.52(8.40)$ \\
$\mathrm{J} 033248-001012$ & $\mathrm{~J} 0332-00$ & 0.310 & 4.55 & $8.50(8.53)$ \\
$\mathrm{J} 033435+003724$ & $\mathrm{~J} 0334+00$ & 0.407 & 5.43 & $8.61(8.75)$ \\
$\mathrm{J} 084856+013647$ & $\mathrm{~J} 0848-01$ & 0.350 & 4.95 & $8.56(8.46)$ \\
$\mathrm{J} 090414-002144$ & $\mathrm{~J} 0904-00$ & 0.353 & 4.98 & $8.93(8.89)$ \\
$\mathrm{J} 092318+010144$ & $\mathrm{~J} 0923+01$ & 0.386 & 5.27 & $8.94(8.78)$ \\
$\mathrm{J} 092356+012002$ & $\mathrm{~J} 0924+01$ & 0.380 & 5.22 & $8.59(8.46)$ \\
$\mathrm{J} 094836+002104$ & $\mathrm{~J} 0948+00$ & 0.324 & 4.71 & $8.52(8.57)$ \\
$\mathrm{J} 130740-021455$ & $\mathrm{~J} 1307-02$ & 0.425 & 5.63 & $8.92(9.01)$ \\
$\mathrm{J} 133735-012815$ & $\mathrm{~J} 1337-01$ & 0.328 & 4.78 & $8.72(8.73)$ \\
\hline
\end{tabular}

$\log \left(L_{[\mathrm{O} \mathrm{III]}} / \mathrm{L}_{\odot}\right)>10^{8.37}$ imposed (to reflect the lowest $L_{\text {[O III] }}$ from the sample according to Reyes et al. 2008), no extra objects would be included from the Reyes et al. (2008) catalogue over those selected from the Zakamska et al. (2003) catalogue using our original selection criteria.

The primary optical spectroscopic data used in this paper were obtained in service mode using the Gemini Multi-Object Spectrograph (GMOS-S) mounted on the $8.1 \mathrm{~m}$ Gemini South telescope at Cerro Pachón, Chile. The GMOS-S detector (Hook et al. 2004) consists of three adjacent $2048 \times 4096$ pixel CCDs separated by two gaps of approximately 2.8 arcsec, and a binned pixel scale of 0.292 (0.146 unbinned) arcsec pixel $^{-1}$.

Long-slit spectra were taken for 18 objects during semesters 2010B and 2011B using a relatively wide slit to enable accurate spectrophotometry. Due to the absence of an atmospheric dispersion compensator on GMOS-S, the objects were mainly observed at low airmass and/or with the slit placed at the parallactic angle, in order to minimize the effects of differential refraction (see Table 2). With the exception of J0332-00, the slit position angles of those objects not observed in the above configuration were within $20^{\circ}$ of the parallactic angles, and were also mostly observed at low to moderate airmasses ( $\mathrm{AM}<1.25)$. Due to our use of a relatively wide slit $(1.5 \operatorname{arcsec})$, differential refraction is unlikely to have affected these observations. The observations were made predominately in dark time (except for J0227+01, J0320+00, J0848+01, J0948+00 and $\mathbf{J} 2358-00$, which were observed in grey time), in photometric conditions. The seeing for each object was measured using the stars in the acquisition images, and in all but one case the seeing was FWHM $<1.15 \operatorname{arcsec}(0.53<$ FWHM $<1.58$ arcsec with a median of 0.81 arcsec FWHM, see Table 2). For most objects, the observations consist of $4 \times 675 \mathrm{~s}$ exposures using the B600 grating with 600 groves $\mathrm{mm}^{-1}$ blazed at $461 \mathrm{~nm}$, and $3 \times 400 \mathrm{~s}$ exposures using the R400 grating with 400 groves $\mathrm{mm}^{-1}$ blazed at $764 \mathrm{~nm}$. The exposures in the two wavelength ranges were interleaved in order to mitigate any changes in the atmospheric conditions during each observational run. The full details of the observations are shown in Table 2.

The data were reduced using a combination of the Gemini GMOS dedicated data reduction package within the IRAF environment, other standard IRAF routines and the STARLINK FIGARO ${ }^{1}$ package. Initially, the three CCD images, which comprise each exposure, were mosaicked into a single image, which was then bias subtracted and flat fielded. The images were then combined using IMCOMBINE (IRAF) to produce the final 2D frame. The wavelength calibration for each image was checked by extracting a 1D spectrum of the sky emission lines close to the target position along the slit, and fitting the position of the most prominent lines using a Gaussian profile. Using this technique, the uncertainty in the wavelength calibration was found to be $\sim 0.3 \AA$ and $\sim 0.5 \AA$ for the B600 and R400 exposures, respectively. The average spectral resolutions measured from the night sky lines were 7.2 and $11.4 \AA$, for the blue and red wavelength ranges, respectively.

Due to the fact that an accurate flux calibration is essential when attempting to model stellar populations, several standard stars were observed over the course of each semester. Flux calibration curves were derived for each star individually, which were then compared to each other (independently for each semester) across the full wavelength range. Clearly outlying curves (i.e. due to misidentification of the standard star, non-photometric conditions, etc.) were rejected before combining the curves ( 5 for 2010B and 3 for 2011B) into an average flux calibration curve, which was then applied to all the objects observed in that semester. From comparison between the individual flux calibration curves, it was found that the accuracy of the relative flux calibration was \pm 5 per cent. There is also a large region of overlap between the red and blue spectra $(\sim 2080 \AA)$, allowing a further check that the calibrations of the spectra taken with the two gratings match well. In most cases, the agreement was excellent, and the red and blue spectra agreed in both shape and level across the full region of the overlap to within 5 per cent. In three cases (J0217-00, J0249+00 and J0924+01), the differences in the flux levels between the blue and red spectra were significant ( 8 per cent, 13 per cent and 22 per cent, respectively), although the shapes of the spectra in all cases agreed well. However, because this occurred in a limited number of cases, it was considered that this was due to changes in the atmospheric conditions between the observations made using the blue and red grisms, rather than being an issue with the flux calibration. Therefore, either the blue or red spectrum was scaled to match its counterpart with the higher continuum flux. The final step was to correct the spectra for any spatial distortions, using the IRAF APALL package. The useful observed wavelength range is 3940-9140 A, although wavelengths longer than $8200 \AA$ are strongly affected by residual fringes.

We were unable to obtain a spectrum of J0114+00 through our Gemini programme. However, its SDSS spectrum is of sufficient quality to enable stellar population modelling. This spectrum was taken using the standard 3 arcsec fibre covering an observed wavelength range of 3800-9200 $\AA$ ( 2700-6620 $\AA$ rest-frame) with a spectral resolution varying from 1500 at $3800 \AA$ to 2500 at $9000 \AA$.

\footnotetext{
${ }^{1}$ http://starlink.jach.hawaii.edu/starlink/WelcomePage.
} 
Table 2. Details of the long-slit spectroscopic observations carried out at Gemini South using the GMOS instrument. Column 1 gives the object name, columns 2 and 3 give the observation date and slit position angle, columns 4 and 5 give the exposure times in seconds using the blue and red grisms, respectively. Column 6 gives the average airmass throughout the exposures and column 7 gives the seeing for the observations in arcseconds as measured from the acquisition image. The final column denotes whether the object was observed at the parallactic angle.

\begin{tabular}{lccccccc}
\hline Name & obs. date & $\begin{array}{c}\text { PA } \\
\left({ }^{\circ}\right.\end{array}$ & $\begin{array}{c}\text { Exposure } \\
\text { Blue(s) }\end{array}$ & $\begin{array}{c}\text { Exposure } \\
\text { Red(s) }\end{array}$ & $\begin{array}{c}\text { Average } \\
\text { airmass }\end{array}$ & $\begin{array}{c}\text { Seeing } \\
(\operatorname{arcsec})\end{array}$ & $\begin{array}{c}\text { Mean } \\
\text { Parallactic? }\end{array}$ \\
\hline J2358-00 & $2011-09-03$ & 331 & $4 \times 675$ & $3 \times 400$ & 1.40 & 1.58 & No \\
J0025-10 & $2010-11-30$ & 230 & $4 \times 675$ & $3 \times 400$ & 1.10 & 0.84 & No \\
J0123+00 & $2011-10-24$ & 209 & $4 \times 675$ & $3 \times 400$ & 1.18 & 1.12 & No \\
J0142+14 & $2011-12-23$ & 155 & $1 \times 6751 \times 461$ & - & 1.57 & 0.73 & Yes \\
J0217-01 & $2010-12-03$ & 146 & $4 \times 675$ & $3 \times 400$ & 1.26 & 0.97 & Yes \\
J0217-00 & $2011-10-23$ & 222 & $4 \times 675$ & $3 \times 400$ & 1.32 & 0.86 & Yes \\
J0218-00 & $2010-12-04$ & 171 & $4 \times 675$ & $3 \times 400$ & 1.17 & 1.11 & Yes \\
J0227+01 & $2011-01-26$ & 138 & $4 \times 675$ & $3 \times 400$ & 1.57 & 0.53 & Yes \\
J0234-07 & $2010-12-06$ & 126 & $4 \times 675$ & $3 \times 400$ & 1.57 & 0.78 & Yes \\
J0249+00 & $2010-12-05$ & 0 & $4 \times 675$ & $3 \times 400$ & 1.17 & 0.73 & Yes \\
J0320+00 & $2010-12-05$ & 216 & $4 \times 675$ & $3 \times 400$ & 1.27 & 0.75 & Yes \\
J0332-00 & $2010-12-06$ & 32 & $4 \times 675$ & $3 \times 400$ & 1.16 & 0.83 & No \\
J0334+00 & $2010-12-01$ & 350 & $4 \times 675$ & $3 \times 400$ & 1.24 & 1.13 & No \\
J0848+01 & $2011-01-25$ & 174 & $4 \times 675$ & $3 \times 400$ & 1.22 & 0.72 & No \\
J0904-00 & $2012-01-20$ & 140 & $4 \times 675$ & $3 \times 400$ & 1.46 & 0.71 & Yes \\
J0923+01 & $2011-11-30$ & 222 & $4 \times 675$ & $3 \times 400$ & 1.37 & 0.67 & Yes \\
J0924+01 & $2011-02-01$ & 0 & $4 \times 675$ & $3 \times 400$ & 1.18 & 0.75 & Yes \\
J0948+00 & $2011-01-25$ & 145 & $4 \times 675$ & $3 \times 400$ & 1.32 & 0.89 & Yes \\
\hline
\end{tabular}

Table 3. Details of the long-slit observations carried out using the OSIRIS instrument on the GTC. Column 1 is the abbreviated object name and column 2 gives the dates of observations. The exposure times in each of the three wavelength ranges are given in columns $3-5$, whilst the average airmass and seeing for the combined observations of each target are given in columns 6 and 7, respectively.

\begin{tabular}{lcccccc}
\hline Name & Obs. date & $\begin{array}{c}\text { Exposure } \\
\text { Blue(s) }\end{array}$ & $\begin{array}{c}\text { Exposure } \\
\text { Yellow(s) }\end{array}$ & $\begin{array}{c}\text { Exposure } \\
\text { Red(s) }\end{array}$ & Airmass & $\begin{array}{c}\text { Seeing } \\
\text { (arcsec) }\end{array}$ \\
\hline J0217-00 & $16 / 17 / 18-08-2012$ & $3 \times 1800$ & $3 \times 1200$ & $3 \times 1800$ & 1.37 & 0.95 \\
J1307-02 & $01 / 13-04-2012$ & $3 \times 1800$ & $3 \times 1200$ & $3 \times 1800$ & 1.21 & 0.95 \\
J1337-01 & $13 / 26-04-2012$ & $3 \times 1800$ & $3 \times 1200$ & $3 \times 1800$ & 1.25 & 1.2 \\
\hline
\end{tabular}

\subsection{Additional type II quasar observations}

Spectroscopic observations of three type II quasars were also taken at the Gran Telescopio Canarias (GTC), La Palma, on 2012 April 1, 13, 26 and 2012 August 16-18. The observations were obtained under good sky conditions (dark nights with clear/photometric transparency), and seeing values ranging from 0.9 to 1.0 arcsec (FWHM) for J0217-00 and J1307-02, and 1.2 arcsec (FWHM) for J1337-01, respectively. The OSIRIS instrument was used in long-slit spectroscopy mode, with each target being observed with the R2000B, R2500R and R2500I grisms for integration times of $3 \times 1800 \mathrm{~s}, 3 \times 1200 \mathrm{~s}$ and $3 \times 1800 \mathrm{~s}$, respectively. A slit of width 1.23 arcsec was used oriented at the parallactic angle to minimize flux losses, giving a spectral resolution (FWHM) of 4, 5 and $6.5 \AA$ in the three respective grisms. The reduction and calibration of these long-slit observations were performed in the standard way, using IRAF routines. Full details of the observations made for these three objects are given in Table 3 . Of these three objects, two (J1307-02 and J1337-01) are in addition to the original sample. Both objects fulfil the original [O III] luminosity selection criteria (Table 1), although J1307-02 is at slightly higher redshift $(z=0.425)$ than the original sample selection. J0217-00 was also observed as part of the original Gemini sample; however, the data presented here are taken at a different position angle, sampling different extended regions of the galaxy, although the flux is likely still dominated by the nuclear regions also sampled by our Gemini spectra.

\section{ANALYSIS}

For the majority of Gemini spectra, only one aperture was extracted from each object, which was centred on the peak continuum flux. In all such cases, the aperture had a metric diameter of $\sim 8 \mathrm{kpc}$ and was centred on the nucleus of each target. However, note that the apertures were not exactly $8 \mathrm{kpc}$ because of the finite pixel scale. The sizes of the apertures were 10-12 pixels, depending on the redshift of the object.

In two cases (J0218-00 and J0332-00), it was possible to extract two apertures, one with a diameter of $8 \mathrm{kpc}$ centred on the nuclear region of the quasar host galaxy $(\mathrm{N})$, and a second aperture extracted from an extended region using a 5-kpc-diameter aperture $(\mathrm{E})$. The physical location of the apertures are shown in Figs A7 (J0218-00) and Fig. A14 (J0332-00).

All spectra were corrected for Galactic extinction using the $E(B-V)$ values of Schlegel, Finkbeiner \& Davis (1998) and the Cardelli, Clayton \& Mathis (1989) extinction law, and were then shifted to the rest frame before stellar synthesis modelling. The spectra for all the sample objects including the SDSS spectrum of J0114+00 are shown in Fig. 1. 

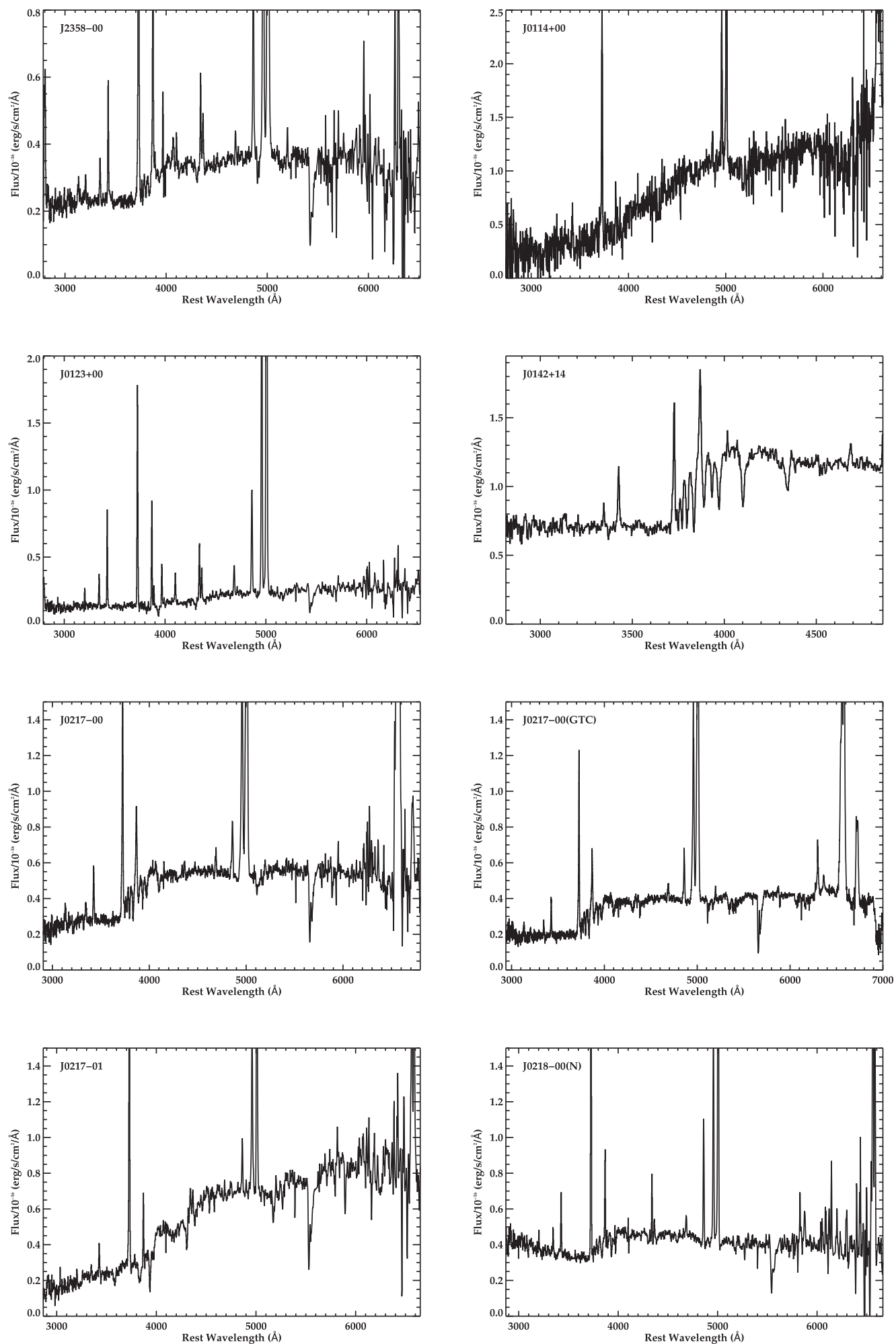

Figure 1. The Gemini GMOS-S spectra for 17 of the objects, the SDSS spectrum of J0114+00 and the 3 GTC spectra of J0217-00, J1307-02 and J1337-01 all shifted to the rest frame. The name of each object is given in each panel.

\subsection{Continuum modelling}

To model the stellar continuum, we used a purpose written IDL routine (CONFIT; Robinson et al. 2000; Robinson 2001), which is capable of fitting up to three components to the observed spectrum, comprising up to two stellar components (one fixed in age and one varying), and additionally, a power-law component of the form $F_{\lambda} \propto \lambda^{\alpha}(-15<\alpha<15)$. The routine fits the specified number of components to the data using varying combinations of flux in each component to compute the reduced minimum $\chi^{2}\left(\chi_{\text {red }}^{2}\right)$ value for each combination. The combinations of components are allowed to 

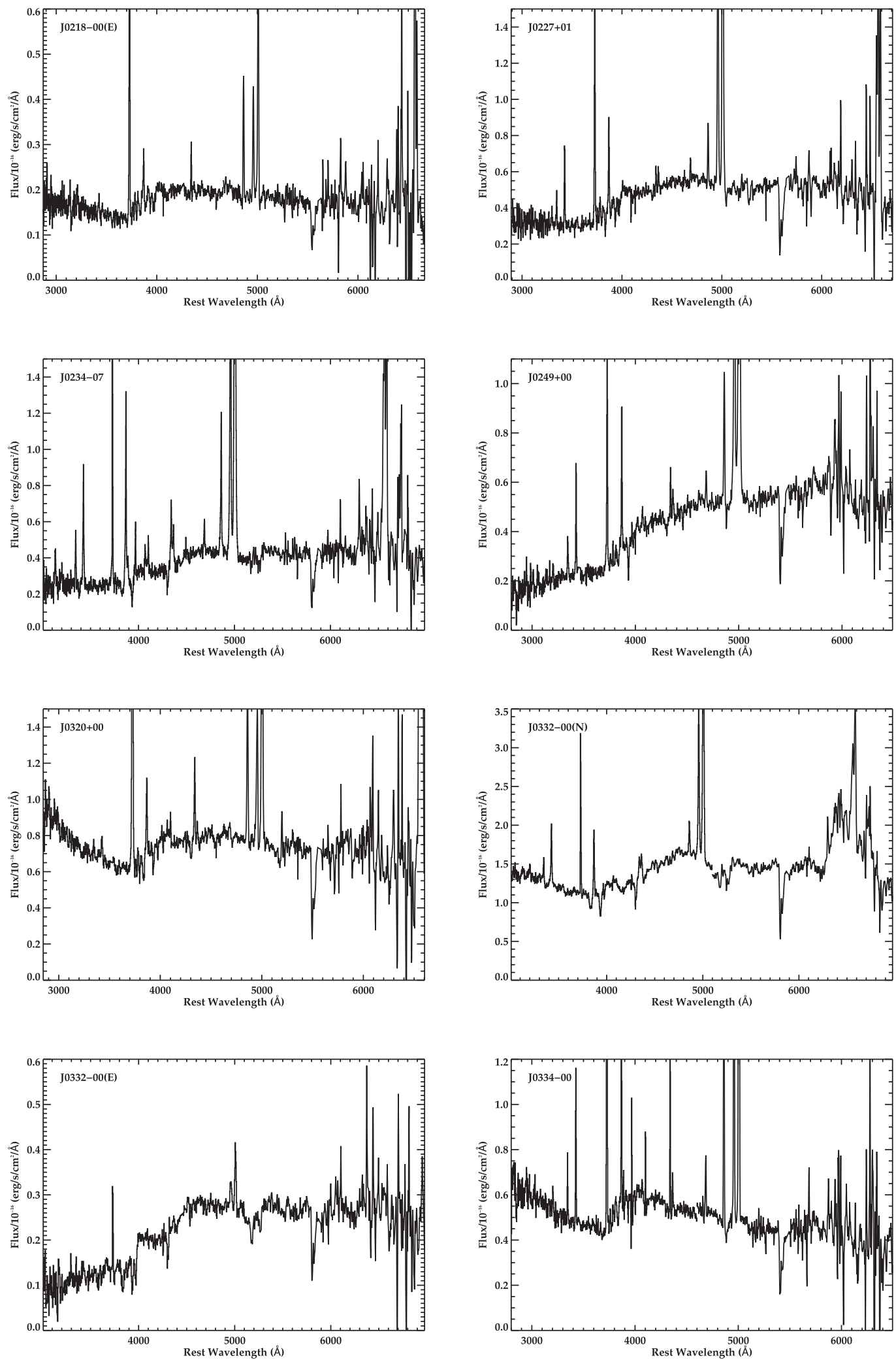

Figure 1 - continued

vary up to 125 per cent of the measured flux in the normalizing bin in order to account for the uncertainties in both the data and the models. Each spectrum was partitioned into a number of wavelength bins $(\sim 70)$, avoiding strong emission lines, chip gaps, dead columns and other cosmic defects. In order to perform the modelling, we selected a normalizing bin in the region of 4400-4800 for each spectrum, and assumed a relative flux calibration error of \pm 5 per cent (as outlined above). CONFIT has been successfully used 

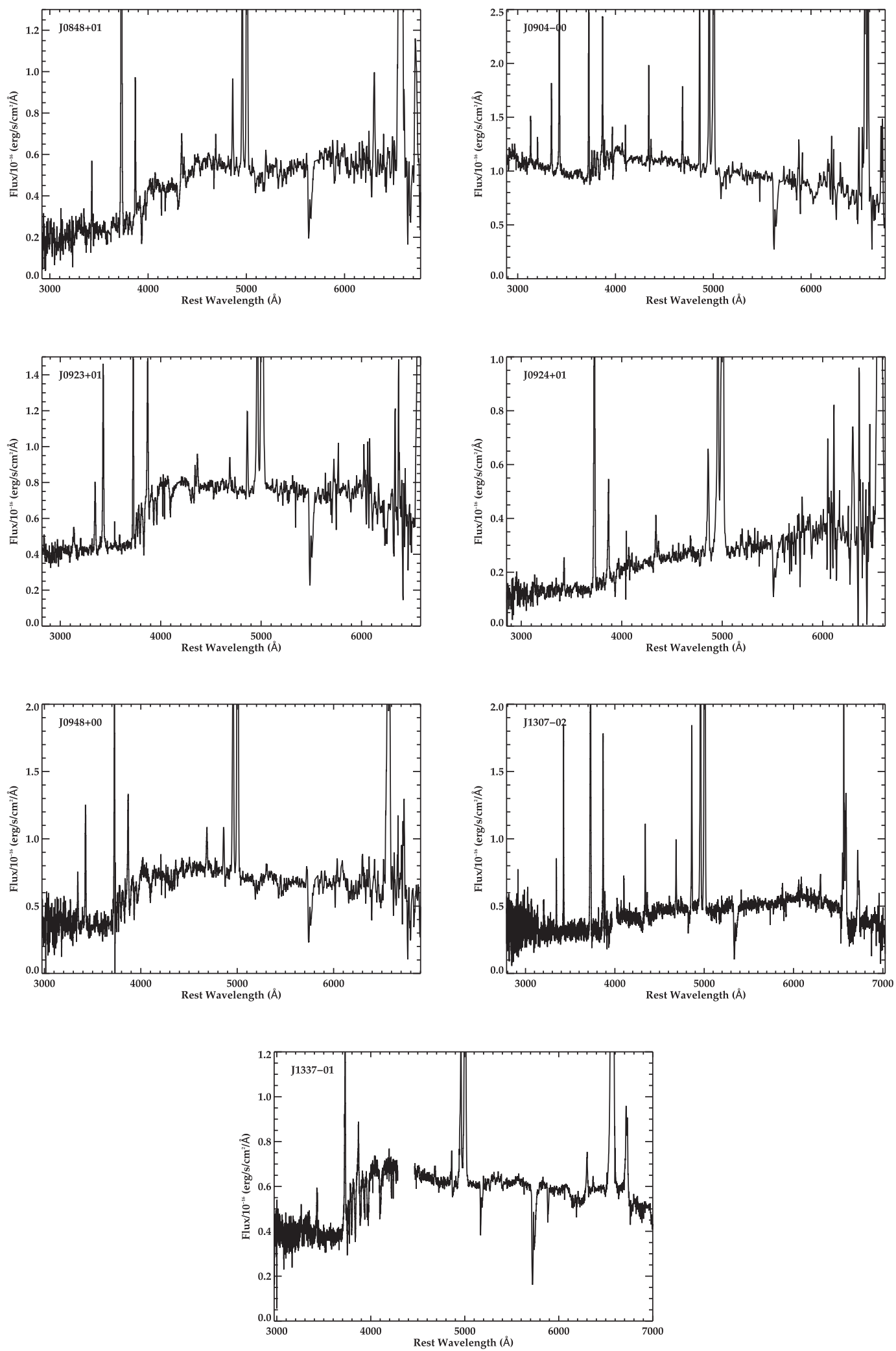

Figure 1 - continued.

to carry out spectral synthesis modelling for galaxies with a wide range of properties. Examples can be found in Holt et al. (2007), Wills et al. (2008) and Rodríguez Zaurín, Tadhunter \& González Delgado (2009).
In order to better fit the stellar populations, the higher order Balmer emission lines and a nebular continuum were subtracted using the procedure outlined in Dickson et al. (1995) and Holt, Tadhunter \& Morganti (2003). The process of generating 
Table 4. The Balmer decrements measured from each of the apertures extracted from the full sample of type II quasars. Column 1 gives the name of the object and if appropriate, which aperture is being referred to. Columns 2, 3 and 4 give the $\mathrm{H} \gamma / \mathrm{H} \beta, \mathrm{H} \delta / \mathrm{H} \beta$ and $\mathrm{H} \alpha / \mathrm{H} \beta$ ratios, respectively, along with the associated fitting error (Case B values: $\mathrm{H} \gamma / \mathrm{H} \beta=0.468, \mathrm{H} \delta / \mathrm{H} \beta=0.259$ and $\mathrm{H} \alpha / \mathrm{H} \beta=2.86$ ). Column 5 gives the percentage contribution of the nebular continuum to the total flux in the wavelength range 3540-3640 $\AA$ and the final column gives the $E(B-V)$ value of reddening applied to the model nebular continuum before subtraction from the data. In the cases in which a reddening correction was applied before subtraction, the value shown in column 5 refers to the percentage of the total flux contributed by the model after the reddening correction has been applied. No value was calculated for $\mathrm{J} 0142+14$ due to the fact that $\mathrm{H} \beta$ fell outside the spectral range.

\begin{tabular}{|c|c|c|c|c|c|}
\hline Name & $\mathrm{H} \gamma / \mathrm{H} \beta$ & $\mathrm{H} \delta / \mathrm{H} \beta$ & $\mathrm{H} \alpha / \mathrm{H} \beta$ & $\begin{array}{l}\text { Nebular continuum } \\
\text { (per cent) }\end{array}$ & $E(B-V)$ \\
\hline $\mathrm{J} 2358-00$ & $0.39 \pm 0.08$ & $0.23 \pm 0.07$ & - & 28 & - \\
\hline $\mathrm{J} 0114+00$ & - & - & - & 14 & - \\
\hline $\mathrm{J} 0123+00$ & $0.43 \pm 0.03$ & $0.22 \pm 0.02$ & - & 20 & 0.2 \\
\hline $\mathrm{J} 0142+14$ & - & - & - & - & - \\
\hline J0217-00 & - & - & - & 14 & - \\
\hline J0217-01 & - & - & - & 20 & - \\
\hline $\mathrm{J} 0218-00(\mathrm{~N})$ & $0.37 \pm 0.02$ & - & - & 17 & - \\
\hline J0218-00(E) & $0.32 \pm 0.02$ & & - & 17 & - \\
\hline $\mathrm{J} 0227+01$ & - & - & - & 11 & - \\
\hline J0234-07 & $0.42 \pm 0.12$ & - & $2.41 \pm 0.12$ & 25 & 0.25 \\
\hline $\mathrm{J} 0249+00$ & $0.35 \pm 0.14$ & - & - & 25 & - \\
\hline $\mathrm{J} 0320+00$ & $0.43 \pm 0.17$ & - & - & 8 & 0.2 \\
\hline $\mathrm{J} 0332-00(\mathrm{~N})$ & - & - & - & 4.1 & - \\
\hline J0332-00(E) & - & - & - & 3.3 & - \\
\hline $\mathrm{J} 0334+00$ & $0.38 \pm 0.02$ & $0.21 \pm 0.01$ & - & 23 & - \\
\hline $\mathrm{J} 0848+01$ & - & - & - & 19 & - \\
\hline J0904-00 & $0.46 \pm 0.17$ & - & $3.82 \pm 1.3$ & 13 & - \\
\hline J0923+01 & - & - & - & 14 & - \\
\hline J0924+01 & $0.33 \pm 0.09$ & - & $4.26 \pm 1.43$ & 14 & 0.3 \\
\hline $\mathrm{J} 0948+00$ & - & - & - & 15 & - \\
\hline $\mathrm{J} 1307-02$ & $0.40 \pm 0.07$ & & - & 27 & - \\
\hline J1337-01 & - & $0.20 \pm 0.04$ & $4.39 \pm 0.7$ & 7.8 & \\
\hline
\end{tabular}

the nebular continuum involved several steps. Initially, the bestfitting stellar model (generated in an initial CONFIT run) was subtracted from the spectrum to account for any underlying stellar absorption of $\mathrm{H} \beta$. Using the STARLINK FIGARO package, the measured $\mathrm{H} \beta$ flux was then used to generate a spiketrum ${ }^{2}$ of the Balmer higher order lines ( $n \geq 8)$, assuming Case $\mathrm{B}$ recombination. This spiketrum was then convolved with Gaussians of the measured FWHM of $\mathrm{H} \beta$, producing a model of the Balmer emission lines.

The next step was to generate a nebular continuum that accounts for the free-free, free-bound and two photon decay continua associated with the nebula. This model was produced using the STARLINK DIPSO package, assuming an electron temperature of $T=10000 \mathrm{~K}$ and electron density of $N_{\mathrm{e}}=10^{2} \mathrm{~cm}^{-3}$. However, it should be noted that the resulting nebular model is not particularly sensitive to the assumed temperature and density. The model of the Balmer emission lines and the continuum nebular emission were then combined to form the final nebular model.

We then attempted to characterize the internal reddening of the galaxy using the Balmer decrement from the stellar subtracted spectrum. Although it would obviously be preferable to measure any reddening of the nebular continuum by using the $\mathrm{H} \alpha / \mathrm{H} \beta$ ratio, the strong fringing at the longest wavelengths in the Gemini spectra

\footnotetext{
${ }^{2}$ A spectrum where all the elements except for those at the tabulated values are zero. In this case, the tabulated values are the expected fluxes of the higher order Balmer lines, considering the measured $\mathrm{H} \beta$ flux.
}

does not allow reliable measurements of the $\mathrm{H} \alpha$ flux. In the case of the higher redshift objects in the sample, $\mathrm{H} \alpha$ is shifted out of the spectral range altogether. Therefore, in order to make some estimate of the reddening of the nebular continuum, the fluxes of the $\mathrm{H} \gamma$ and $\mathrm{H} \delta$ lines were also measured. However, due to the lower equivalent widths of the lines, in some cases the errors were larger than the value of the ratio. In such cases, the values were disregarded (Table 4).

For the purpose of maintaining a consistent approach throughout, a maximum nebular subtraction (i.e. applying no reddening to the model nebular continuum) was attempted first. The resulting nebular subtracted spectrum was then inspected in order to determine if this resulted in a non-physical step around the region of the Balmer edge $(3646 \AA)$. If this was the case, reddening that was consistent with the Balmer decrement measured from the stellar-subtracted spectrum was applied using the Seaton (1979) extinction law. However, in the cases where no Balmer decrement could be measured, no reddening was applied to the model nebular continuum. In all cases where this was necessary, this procedure produced an acceptable result, with no clear oversubtraction at the Balmer edge. This is not unexpected, because the fact that the higher order Balmer emission lines are too weak to measure accurately implies a weaker nebular continuum. Inspection of Table 4 demonstrates that, when the nebular continuum contributes $<20$ per cent of the flux below the Balmer edge, it was not possible to measure the Balmer decrements. This weaker nebular continuum means that the result of the subtraction will not be too sensitive to any reddening correction applied. 
The results presented in column 5 of Table 4 make it clear that it is important to consider the effects of the nebular continuum before embarking on the modelling process. In the vast majority of cases, the nebular continuum accounts for over 15 per cent (ranging from 3 to 28 per cent) of the total flux just below the Balmer edge, measured in the wavelength range 3540-3640 $\AA$ (where the contribution from the nebular continuum will be most pronounced). Failure to account for this component could lead to underestimations of the true ages of the YSPs, because the stellar spectrum will appear bluer than it actually is. The proportions of the UV flux attributed to the nebular continuum in these type II quasars host galaxies are in good agreement with those found for the $2 \mathrm{Jy}$ sample of powerful radio galaxies (PRGs; Tadhunter et al. 2002), where values also ranging from 3 to 28 per cent, were found, with the majority of the sample showing nebular contributions above 15 per cent over the same wavelength range.

In order to assess the effect of the uncertainties in the nebular continuum subtraction on the modelling results, we have carried out the stellar synthesis modelling of a number of objects with differing YSP ages (J0234-07, J0320+00, J0848+01 and J0948+00) varying the degree of reddening applied to the nebular continuum before subtraction by $E(B-V) \pm 0.1$. The results produced by these tests conform to those which we would expect. When the reddening applied to the nebular continuum is increased (i.e. less flux is subtracted in the blue part of the spectrum), we see a slight shift to younger ages with slightly higher reddening for the YSP. These shifts tend to extend the YSP age range by $\sim 1-10$ Myr (depending on the increment available in YSP ages due to the input models), and we find a corresponding increase of $E(B-V)=0.1$ at the youngest ages. Decreasing the reddening (i.e. subtracting more flux in the blue part of the spectrum) results in the same behaviour but in the reverse direction, with the ages increasing and the reddening decreasing at the youngest ages. This confirms than an error in the reddening applied to the nebular continuum of $E(B-V) \sim$ 0.1 will not have any significant effect on the range of YSP ages and reddenings determined for each object, and will not impact the conclusions drawn in this work.

\subsection{Modelling strategy}

The stellar population models used here were generated using the STARBURST99 code of Leitherer et al. (1999), assuming an instantaneous burst star formation history, solar metallicity and a Kroupa initial mass function (Kroupa 2001). Reddening in the range $0 \leq$ $E(B-V) \leq 2.0$ in steps of $\Delta E(B-V)=0.1$ was then applied to these template spectra using the extinction curve of Calzetti et al. (2000), which is appropriate in the starburst case. To carry out the modelling, we used several different components to account for varying scenarios. In all cases, we assumed that there is an underlying older stellar population of either 8 Gyr (old stellar population; OSP) or 2 Gyr (intermediate stellar population; ISP), and in the majority of cases, we also assumed that there is a YSP component. We chose an 8 Gyr underlying population because this allows for the formation of a population of stars at high redshift. However, because there is very little evolution in the spectra of a simple stellar population at large ages, the modelling is not particularly sensitive to the exact age chosen (e.g. 8 Gyr versus 12 Gyr).

The 2 Gyr population was used to account for the possibility that the dominant underlying population of stars was formed in more recent times (e.g. in a previous major merger event) and was chosen because experimentation with different possibilities (i.e. $1 \mathrm{Gyr}$ and
$0.5 \mathrm{Gyr}$ ) showed that the 2 Gyr population produced significantly better results in general. In contrast to the YSP, which are assumed to be the product of a merger-induced, dusty, nuclear starburst, these older populations are assumed to be unreddened because it is probable that these stars are distributed throughout the host galaxy, and that those which contribute to the observed spectra are foreground stars.

It was also possible to include a power-law component in the model, the purpose of which is to account for the possibility of scattered light from the hidden quasar. Although these are type II quasars, it is possible that scattered light may make a significant contribution to observed spectrum (Tadhunter et al. 2002; Obied et al. 2016), and thus must be properly accounted for. For the purposes of the modelling, five combinations of components, outlined below, were adopted, which reflect plausible combinations of the various components that contribute to the observed spectra. ${ }^{3}$

(1) An unreddened, 8 Gyr OSP combined with a power-law component, accounting for the case of an OSP plus a scattered or direct AGN component but no YSP.

(2) An unreddened, 8 Gyr underlying population, combined with instantaneous burst star formation models, with ages $0.001<$ $t_{\mathrm{YSP}}(\mathrm{Gyr})<5.0$ and reddening $0 \leq E(B-V) \leq 2.0$.

(3) An unreddened, 2 Gyr underlying population, combined with instantaneous burst star formation models, with ages $0.001<t_{\mathrm{YSP}}(\mathrm{Gyr})<2.0$ and reddening $0 \leq E(B-V) \leq 2.0$.

(4) The same as 2 with the addition of a power-law component.

(5) The same as 3 with the addition of a power-law component.

In all cases, the overall shape, higher order Balmer lines, Ca II $\mathrm{K}$ and $G$-band fits for solutions that produce $\chi_{\text {red }}^{2}<1$ were visually examined to determine if they were acceptable. When any of these age-sensitive regions produce a very poor fit, the combination of components was rejected as an acceptable solution. In cases where a large number of combinations produce $\chi_{\text {red }}^{2}<1$, a representative sample of combinations was examined, taking account of the allowed values of YSP/ISP ages and associated reddenings. For any combination used, it is possible that more than one minima in $\chi_{\text {red }}^{2}$ will be produced (see Fig. 2 for an example). An example of what we consider to be an acceptable fit is shown in Fig. 3. The top panel shows the overall fit to the spectrum (red), with the individual contributions for the OSP (8 Gyr) and YSP (0.08 Gyr) shown in green. The residuals of the fit are shown along the bottom for each bin. The bottom panel shows a zoom-in of the Balmer absorption lines, which, in this example, are prominent in the spectrum. This fit assumes combination 2 and therefore, no power-law component is included.

The exception to the method of inspection outlined above was J0332-00, because it has subsequently become apparent that it is actually a type I object in which the broad Balmer emission lines are directly detected (see Appendix A12). In this case, because of the strong power-law component, for combination 4 all combinations produced $\chi_{\text {red }}^{2}<1$ while for combination 5, large sections of parameter space produced $\chi_{\text {red }}^{2}<1$. Therefore, we are not able to offer any meaningful constraint to the age of the YSP (if any) in this object.

\footnotetext{
${ }^{3}$ We do not include an 8 Gyr population combined with a 2 Gyr population and a YSP, which would account for more than one previous episode of star formation, because it would be very difficult to disentangle the contribution of the $8 \mathrm{Gyr}$ population from that of the $2 \mathrm{Gyr}$ population.
} 


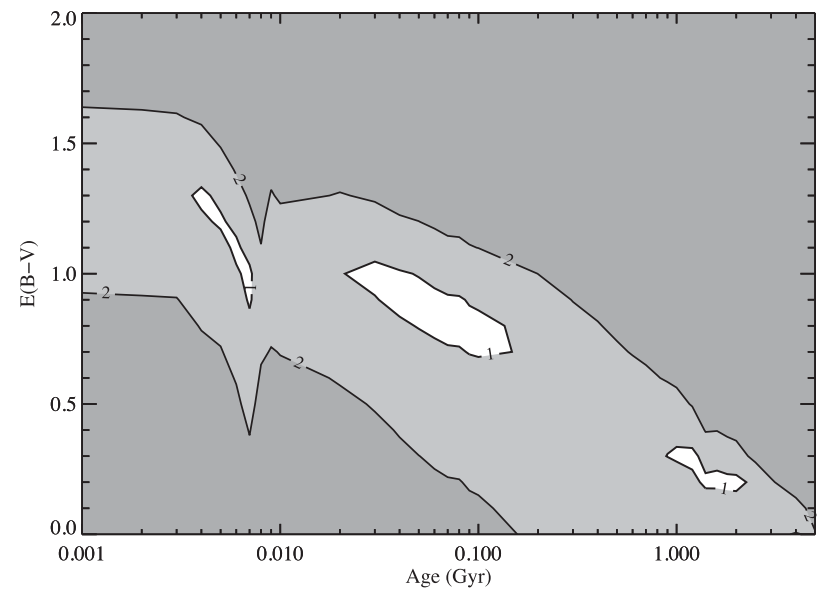

Figure 2. The $\chi_{\text {red }}^{2}$ plot produced from modelling J0217-01 assuming combination 2. The plot shows the age of the YSP against the reddening of the YSP. The areas shown in white are the regions in which the modelling produced $\chi_{\text {red }}^{2}<1$. This example shows that, in this particular case, three distinct regions produced such values.
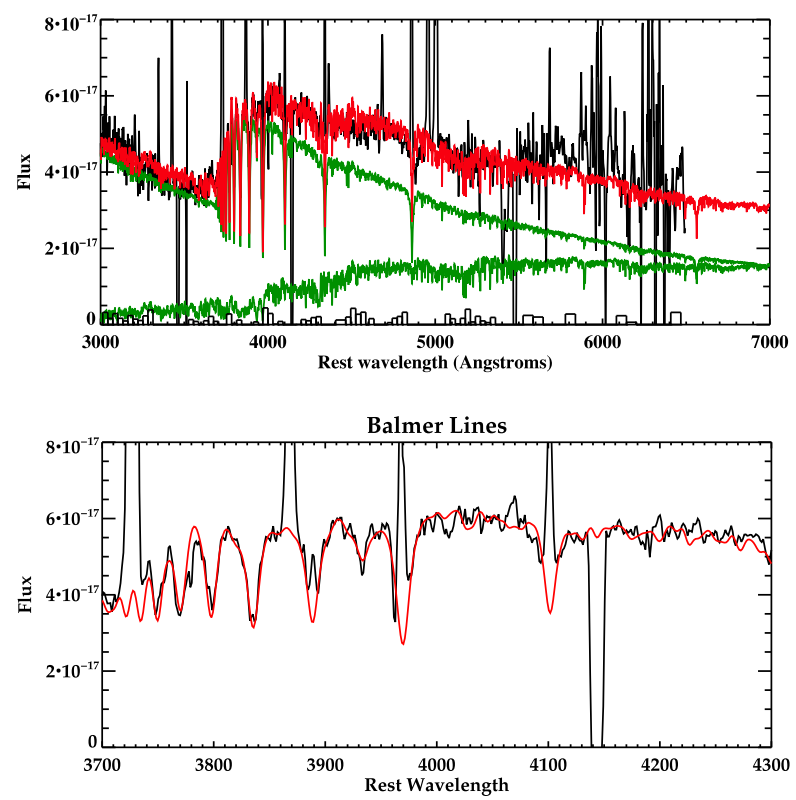

Figure 3. An example of an acceptable fit produced by CoNFIT for J0334+00. The fit shown here was produced using combination 2 and includes a YSP with $t_{\mathrm{YSP}}=0.08 \mathrm{Gyr}$ and $E(B-V)=0$. The top panel shows the overall fit to the spectrum (red) while the individual components are shown in green. The residual for the fit are shown at the bottom. The bottom panel shows a zoom in of the region including the higher order Balmer lines. The solid black line shows the data while the red line shows our fit. The large drop in the continuum wavelengths $\sim 4140-4150 \AA$ is not intrinsic to the galaxy, but due to a gap between two of the CCD detectors.

\section{RESULTS}

\subsection{The effect of including a power-law component}

The significance of the power-law component, particularly in objects that are well fitted by combinations that do not require the inclusion of one, remains one of the largest sources of uncertainty in this work. To unambiguously detect a scattered quasar component would require spectropolarimetry data, but it should be noted that in all but one case ( $\mathrm{J} 0332-00)$, it is possible to fit the $\mathrm{H} \beta$ line using the same kinematic components as those used for [O III] $] \lambda \lambda 4959,5007$, which are, or course, produced in the narrow-line region. As we show next, this strongly suggests that any contamination from scattered quasar light is relatively small because no broad $\mathrm{H} \beta$ component $\left(\mathrm{FWHM}>2000 \mathrm{kms}^{-1}\right.$ ) is clearly visible in the stellarsubtracted spectrum for any of the objects presented here (except the type I object J0332-00). However, it is important to consider the effect that introducing a power law has on the results of the stellar population modelling. Therefore, an attempt to place an upper limit on the power-law contribution to the total flux for each object, using the information yielded by the stellar synthesis modelling, has been made.

One aspect of the stellar synthesis modelling that is common between all the objects is that, when a power-law component is included in the fits, the maximum age that produces solution with $\chi_{\text {red }}^{2}<1$ increases significantly, and the range of reddenings allowed is also extended. In both the cases of objects with increasing and decreasing flux towards the UV, the age of the YSP generally increases with increasing power-law contribution. The reasons for these trends are illustrated in Fig. 4, which shows examples of combination 4 fits for the minimum and maximum cases of power-law flux in the normalizing bin. The top two panels show examples of fits for J0924+01, which has one of the most strongly reddened YSPs of the objects in the sample. The left-hand panel shows the minimum power-law contribution allowed by combination 4 ( 0 per cent), whilst the right shows the maximum contribution (54 per cent). In both cases, the OSP flux is only a small percentage of the total, and thus does not have a large effect on the overall shape of the stellar model in either case. However, it is clear that in the maximum case, the power-law mimics the shape of the reddened YSP from the minimum case.

The bottom two panels show the same thing but for $\mathrm{J} 0320+00$, which is one of the objects that increases strongly in flux towards the UV. Again the left-hand panel shows the minimum power-law case produced by combination 4 ( 0 per cent), and the right-hand panel shows the maximum power-law case ( 38 per cent). Once again, it is clear that in the maximum case, the power-law component assumes the general shape of the unreddened YSP from the minimum case, thus allowing older YSPs to provide acceptable fits, as they are not required to contribute as strongly to the overall shape of the continuum.

The fact that the significance of the power-law component has a dramatic impact on the maximum ages allowed by the modelling means that it is important to be able to constrain the amount of the total flux attributable to it. Unfortunately, it is not possible to do this directly from the results of our stellar synthesis modelling. However, using the technique outlined in Bessiere et al. (2014), we have made an attempt to place an upper limit on the possible contribution of the power law to the flux in the normalizing bin.

To recap, it was assumed that the percentage contributions and the power-law indices $(\alpha)$ for every acceptable combination of components (using both combinations 4 and 5) are representative of scattered or directly transmitted quasar light. These contributions were then extrapolated from the normalizing bin to the wavelength of $\mathrm{H} \beta$ (using the corresponding value of $\alpha$ ), where Gaussians were constructed using average values of equivalent width and FWHM (78 $\AA$ and $5000 \mathrm{kms}^{-1}$, respectively ${ }^{4}$ ) for the broad $\mathrm{H} \beta$ line in quasars of similar luminosity. This produced models for the $\mathrm{H} \beta$ component

\footnotetext{
${ }^{4}$ These values were derived from 'A Catalog of Quasar Properties from
} SDSS DR7' (Schneider et al. 2010; Shen et al. 2011), by selecting all 

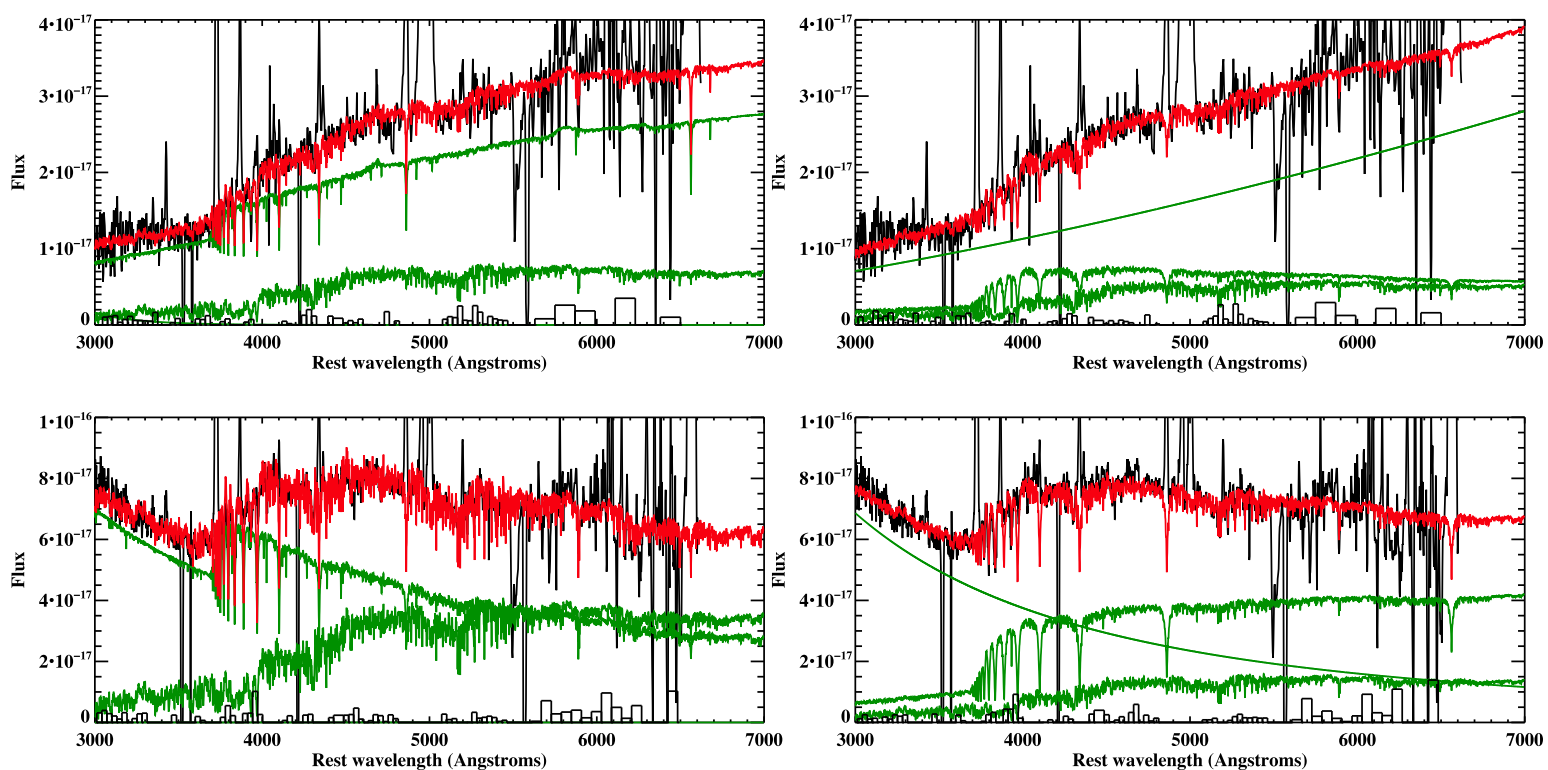

Figure 4. Examples of how the power-law contribution is related to the age and reddening of the YSP. The top-left panel shows a fit for J0924+01, which incorporates only an OSP and YSP. The fit shows a YSP of $t_{Y S P}=0.005 \mathrm{Gyr}$ and $E(B-V)=1.0$. The top-right panel shows the same object but with the maximum allowable power law of $\sim 50$ per cent included in the fit. In this case, $t_{Y S P}=0.5 \mathrm{Gyr}$ with $E(B-V)=0.3$ The bottom panels show the same but for $\mathrm{J} 0320+00$, which has a maximum allowable power-law component of $\sim 40$ percent. The left-hand panel shows a YSP with $t_{\mathrm{YSP}}=0.02 \mathrm{Gyr}$ and $E(B-V)=$ 0.2 whilst the right-hand panel shows a YSP with $t_{\mathrm{YSP}}=0.3$ and $E(B-V)=0.6$.

generated in the broad-line region (BLR), which would be present as a scattered component at a given level of power-law flux. As the power-law flux increases, this broad $\mathrm{H} \beta$ component should, at some point, become detectable. In order to test this, broad $\mathrm{H} \beta$ models were constructed assuming different levels of power-law contribution to the total flux, for each object independently (increasing in steps of 5 per cent up the maximum allowed by the modelling). Each level of power-law contribution is associated with a particular stellar model, consisting of a OSP and a YSP. For each level of power-law contribution, the stellar model corresponding to the power-law model was subtracted from the nebular subtracted data. Each Gaussian model was then combined with its power-law component, and then subtracted in turn from their corresponding stellar-subtracted spectrum in an attempt to quantify the point at which the oversubtraction of the continuum becomes clearly detectable on visual inspection.

Fig. 5 shows a zoom of the stellar-subtracted spectrum for each object. In each case, the solid black line shows the data with both the stellar model and the nebular continuum subtracted, the dashed red line denotes the 'just detected' broad $\mathrm{H} \beta$ model (comprising the Gaussian and power-law component) that was subtracted from the spectrum. In the cases in which the subtraction of the broad $\mathrm{H} \beta$ model, associated with the maximum possible contribution to the total flux by the power-law component, does not result in a visible oversubtraction of the continuum, Fig. 5 shows this maximum broad $\mathrm{H} \beta$ case (green dashed line). In the cases where the oversubtraction of the continuum becomes clearly detectable below the maximum value allowed by the modelling, a constraint is placed on the maximum contribution of the power law to the total flux in the normalizing bin. Where this is the case, a plot of the stellar-subtracted spectrum in which it has been determined that the

objects that have $L_{\mathrm{O} \text { III }}>10^{42} \mathrm{erg} \mathrm{s}^{-1}$ and taking the median values of broad $\mathrm{H} \beta$ equivalent width and FWHM. subtraction of the broad $\mathrm{H} \beta$ model has just become clearly visible is shown (blue dashed line).

Taking these additional constraints on the power-law contribution into account, the results of our stellar synthesis modelling are shown in Tables 5 and 6 . Table 5 shows the results for combinations including an 8-Gyr-old underlying population, and Table 6 shows the results if we assume an underlying population of $2 \mathrm{Gyr}$.

It should be noted that the fitted power law does not necessarily represent scattered AGN light, and may instead be associated with a very young stellar population (VYSP). However, it is not possible to clearly distinguish between the two possibilities on the basis of the data presented here because a VYSP $(<10 \mathrm{Myr})$ has only weak age associated features such as the Balmer break and stellar absorption features, so that such features will not contribute strongly to an OSP + VYSP composite. Therefore, to properly constrain the power-law component, not only in terms of its contribution to the observed flux and its spectral index, but also to distinguish between a scattered AGN component and VYSP, spectropolometric observations are essential. This information will allow more stringent constraints to be placed on the ages, reddenings and contributions to the total flux for any YSP/ISP component present in the host galaxies of these type II quasar.

\subsection{The incidence of YSPs in type II quasars}

One of the most obvious features in an optical spectrum, which can be considered an unambiguous indicator of a strong YSP component, is the detection of the higher order Balmer absorption lines. Taking into consideration the potential uncertainties with the subtraction of the higher order Balmer emission lines, the proportion of type II quasar host galaxies (including J0025-10) that show Balmer lines in absorption before performing the nebular subtraction was first determined. In this case, it was found that the Balmer absorption lines are detected in 13/21 spectra ( $\sim 62$ per cent). Following subtraction of the higher order Balmer lines (in emission) 

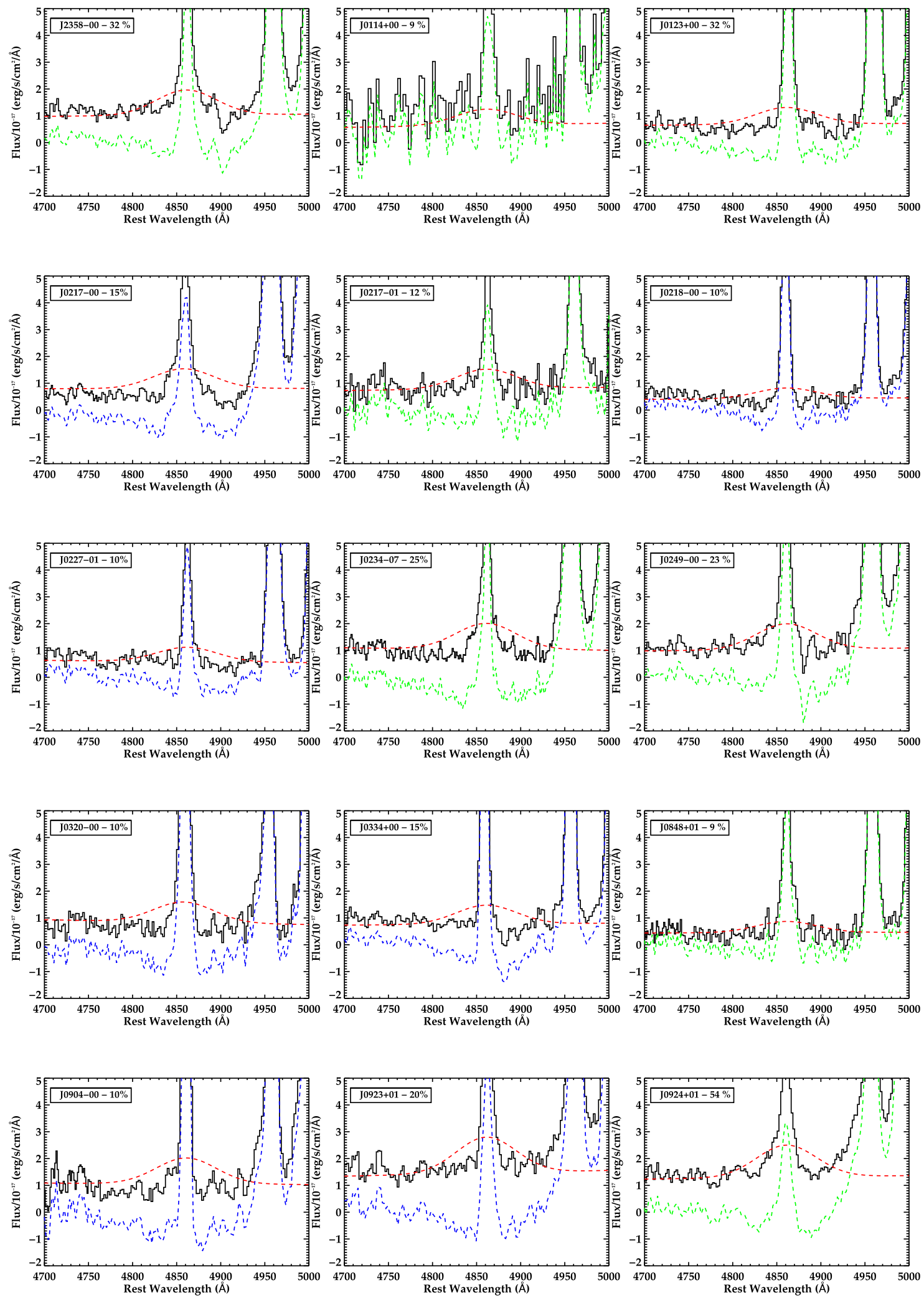

Figure 5. A zoom of the stellar subtracted spectrum in the region around the $\mathrm{H} \beta$ line is shown for each object. The solid black line shows the stellar subtracted data, whilst the dashed red line shows the broad $\mathrm{H} \beta$ plus power-law model that has been subtracted from these data. The blue or green dashed line shows the result of the subtraction (explanation in text). The percentage contribution of the power-law to the total flux in the normalizing bin from which the broad $\mathrm{H} \beta$ model has been constructed is shown in each panel. 

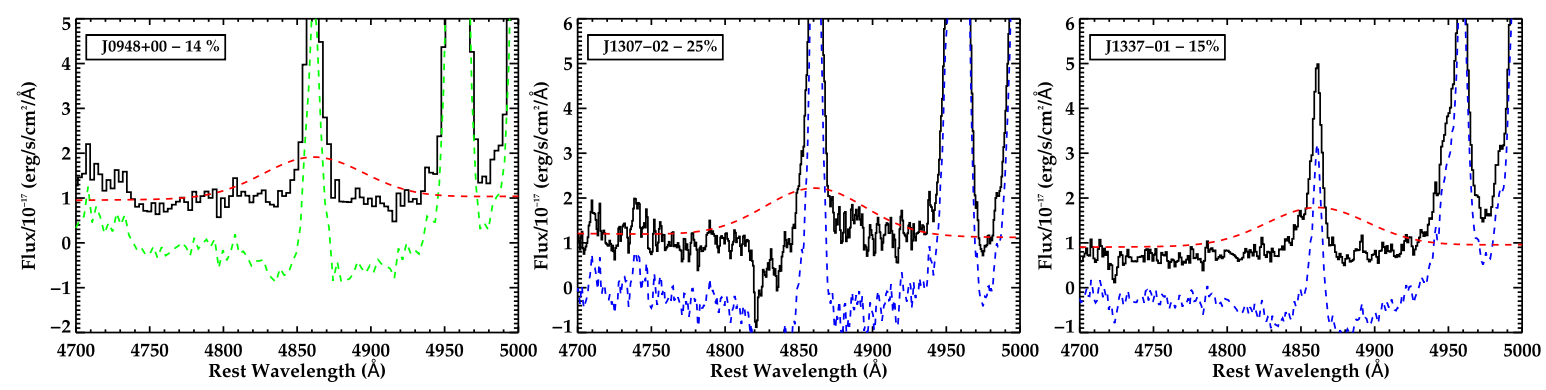

Figure 5 - continued

and nebular continuum, this proportion increased to $14 / 21$ objects ( $\sim 67$ per cent).

In order to constrain the proportion of type II quasars that contain YSPs more accurately, it is also instructive to consider what percentage require a YSP component in order to produce an acceptable fit to their spectral energy distributions, that is, the proportion that cannot be fitted with a power-law +8 Gyr model (combination 1) alone (based on our $\chi_{\text {red }}^{2}<1$ criterion). In this sample, only J0234-07 and the type I object J0332-00 can be adequately fit using only an 8 Gyr OSP combined with a power-law component. In all of the remaining cases, this combination of components failed to produce a reasonable fit to the shape of the spectrum around the Balmer break. Therefore, 19/21 (90 per cent) objects require a YSP in order to fit their overall spectral shape, thus allowing the unambiguous statement that YSPs are present in at least 90 per cent of this sample of type II quasars.

\subsection{The ages of the YSPs in type II quasar host galaxies}

The results presented in Tables 5 and 6 show that the assumptions made in the different modelling combinations can have a profound effect on the results produced. Combinations 4 and 5, both of which include a YSP/ISP as well as a power-law component, are successful in producing acceptable fits. Combination 4 ( $\left.t_{\mathrm{OSP}}=8 \mathrm{Gyr}\right)$ was successful in $23 / 23$ (100 per cent) of the apertures to which it was applied, whilst combination $5\left(t_{\mathrm{ISP}}=2 \mathrm{Gyr}\right)$ produced successful fits for 20/23 of the apertures ( 87 per cent). However, given the increased number of free parameters involved, these modelling combinations do not provide any strong constraints on either the ages or the reddenings of the YSP/ISP in the majority of cases (as discussed in Section 4.1).

In light of the above considerations, the remainder of this work will focus on the models that do not include a power-law component. This is justifiable because it is these combinations that give the best constraints on the YSP age, and also allow for direct comparison with other samples of AGN that have taken a similar modelling approach. ${ }^{5}$ Fig. 6 shows age in Gyr plotted against $E(B-V)$ for combinations 2 and 3. For each object, if the modelling for a given combination produced only one region in which $\chi_{\text {red }}^{2}<1$, that value is plotted in black. In the cases where the modelling for a given combination produced two regions of interest, the values for each region are plotted in the same colour. The apertures that were extracted from companion objects or extended regions, rather than the quasar host galaxy nuclei are plotted as triangles. For each

\footnotetext{
${ }^{5}$ However, it must be noted that the conclusions drawn would be significantly altered if a power-law component proved to contribute substantially to the total flux.
}

point, the bars show the extent of the acceptable ranges of age and reddening, whilst the points are plotted at the median.

The top panel of Fig. 6 summarizes the results for combination 2, which produced successful results for $22 / 25$ ( $\sim 88$ per cent) of the apertures to which it was applied. However, it must be noted that J0217-00 is duplicated in these results, but because it can be clearly seen from Tables 5 and 6 that the results from both data sets are consistent with each other, to avoid confusion we will only consider one set of results (the Gemini GMOS-S results) in further discussion. Strikingly, of the 24 unique apertures that are adequately fit by this combination, 18 require the inclusion of a YSP component with $t_{\max } \leq 100 \mathrm{Myr}$, while the majority of acceptable fits for one object (J0114+00) also include a YSP with $t_{\mathrm{YSP}} \leq 100 \mathrm{Myr}$. It is only in two cases (J0848+01 and J0948+00) that a YSP $t_{\mathrm{YSP}}>200 \mathrm{Myr}$ is required in order to achieve acceptable results.

Considering only the unique objects in the sample, the top panel of Fig. 6 shows that a wide range of reddenings is found, although there does not appear to be any correlation between the YSP age and the reddening associated with the population. If those objects that have stellar populations with average reddenings $E(B-V)>0.4$ are considered to be strongly reddened, then $8 / 21$ (38 per cent) of the apertures fall into this category, whilst $13 / 21$ (62 per cent) are not strongly reddened, of which 38 per cent have mean $E(B-V) \leq 0.1$.

The bottom panel of Fig. 6 summarizes the results for combinations 3 , which produced successful fits for 18/25 ( $\sim 72$ per cent) of the apertures to which it was applied. Again, when considering only unique objects, comparing the two panels of Fig. 6 shows that, when an underlying population of $t_{\mathrm{ISP}}=2 \mathrm{Gyr}$ is assumed, a higher proportion of the objects for which fits are achieved have $t_{\max }>100 \mathrm{Myr}(4 / 17)$ than in the case where an $t_{\mathrm{OSP}}=8 \mathrm{Gyr}$ $(3 / 21)$ is assumed ( 24 per cent versus 14 per cent), although this difference is marginal. Once again, if YSPs with $E(B-V)>0.4$ are categorized as significantly reddened, then $9 / 17$ (53 per cent) of the apertures modelled have a significant average reddening. This proportion is higher than that found for combination 2, which is not unexpected because a significantly reddened YSP can mimic an unreddened OSP in the overall shape of its spectrum. However, it must be noted that for both combinations 2 and 3, the majority of the objects require the inclusion of a YSP $t_{\mathrm{YSP}}<100 \mathrm{Myr}$ in order to adequately fit their spectra.

\section{HOW DO TYPE II QUASARS COMPARE WITH OTHER ACTIVE SYSTEMS?}

\subsection{Comparison with PRGs}

When considering the properties of YSPs in the host galaxies of type II quasars, it is important to consider them in the wider context 
Table 5. The results of our stellar synthesis modelling assuming an $8 \mathrm{Gyr}$ underlying stellar population. Columns 1 and 2 give the object name and aperture (N: nuclear; E: Extended; G: companion galaxy). Column 3 shows whether the higher order Balmer absorption lines were detected in the unsubtracted spectrum. Column 4 gives the nebular continuum contribution in the wavelength range 3540-3640 , whilst Column 5 indicates whether a power-law component was included in the model. Columns 6 and 7 show the range of percentage contributions to the total flux of both the OSP and YSP, whilst columns 7 and 8 show the range of ages and reddenings that are acceptable for the YSPs. Finally, column 8 gives the range of percentage contribution by a power-law component (if included).

\begin{tabular}{|c|c|c|c|c|c|c|c|c|c|}
\hline Name & Aperture & $\begin{array}{l}\text { Balmer } \\
\text { lines? }\end{array}$ & $\begin{array}{c}\text { Nebular } \\
\text { (per cent) }\end{array}$ & PL? & $\begin{array}{c}\text { OSP } \\
\text { (per cent) }\end{array}$ & $\begin{array}{c}\text { YSP } \\
\text { (per cent) }\end{array}$ & $\begin{array}{l}\text { YSP age } \\
\text { (Gyr) }\end{array}$ & $E(B-V)$ & $\begin{array}{r}\text { Power law } \\
\text { (per cent) }\end{array}$ \\
\hline \multirow[t]{2}{*}{$\mathrm{J} 2358-00$} & $\mathrm{~N}$ & $\mathrm{Y}$ & 28 & $\mathrm{~N}$ & $0-19$ & $88-95$ & $0.04-0.05$ & $0.5-0.6$ & - \\
\hline & & & & $\mathrm{Y}$ & $0-28$ & $44-100$ & $0.04-0.2$ & $0.2-0.6$ & $0-41$ \\
\hline \multirow[t]{2}{*}{ J0025-10 } & $\mathrm{N}$ & $\mathrm{Y}$ & 3 & $\mathrm{~N}$ & $19-37$ & $63-79$ & $0.02-0.04$ & $0-0.2$ & - \\
\hline & & & & $\mathrm{Y}$ & $0-39$ & $37-79$ & $0.02-0.3$ & $0-0.5$ & $4-39$ \\
\hline J0025-10 & G & $\mathrm{Y}$ & 10 & $\mathrm{~N}$ & - & - & - & - & - \\
\hline J0025-10 & $\mathrm{E}$ & $\mathrm{Y}$ & 9 & $\mathrm{~N}$ & $5-20$ & $77-92$ & $0.03-0.04$ & $0-0.1$ & - \\
\hline \multirow[t]{4}{*}{ J0114+00 } & $\mathrm{N}$ & $\mathrm{N}$ & 14 & $\mathrm{~N}$ & $53-82$ & $17-44$ & $0.004-0.007$ & $0.8-1.3$ & - \\
\hline & & & & $\mathrm{N}$ & $51-81$ & $19-48$ & $0.02-0.4$ & $0.6-1.0$ & - \\
\hline & & & & $\mathrm{Y}$ & $50-82$ & $17-47$ & $0.004-0.007$ & $0.9-1.3$ & $0-9$ \\
\hline & & & & $\mathrm{Y}$ & $47-84$ & $8-51$ & $0.03-0.2$ & $0.3-1.1$ & $0-9$ \\
\hline \multirow{3}{*}{$\mathrm{J} 0123+00$} & $\mathrm{~N}$ & $\mathrm{~N}$ & 20 & $\mathrm{~N}$ & - & - & - & - & - \\
\hline & & & & $\mathrm{Y}$ & $52-81$ & $3-26$ & $0.001-0.01$ & $0.0-0.2$ & $12-40$ \\
\hline & & & & $\mathrm{Y}$ & $0-88$ & $1-96$ & $0.001-5$ & $0.2-2.0$ & $2-35$ \\
\hline \multirow[t]{2}{*}{ J0142+14 } & $\mathrm{N}$ & $\mathrm{Y}$ & - & $\mathrm{N}$ & $2-32$ & $72-98$ & $0.05-0.1$ & $0.2-0.4$ & - \\
\hline & & & & $\mathrm{Y}$ & $0-20$ & $52-100$ & $0.04-0.4$ & $0.1-0.5$ & $0-48$ \\
\hline \multirow{4}{*}{ J0217-00 } & $\mathrm{N}$ & $\mathrm{Y}$ & 14 & $\mathrm{~N}$ & $0-26$ & $72-100$ & $0.06-0.1$ & $0.5-0.6$ & - \\
\hline & & & & $\mathrm{Y}$ & $0-37$ & $55-100$ & $0.06-0.3$ & $0.1-0.6$ & $0-15$ \\
\hline & GTC & $\mathrm{Y}$ & & $\mathrm{N}$ & $0-35$ & $69-100$ & $0.04-0.1$ & $0.4-0.6$ & - \\
\hline & & & & $\mathrm{Y}$ & $0-35$ & $56-99$ & $0.04-0.2$ & $0.2-0.6$ & $0-15$ \\
\hline \multirow[t]{4}{*}{ J0217-01 } & $\mathrm{N}$ & $\mathrm{N}$ & 20 & $\mathrm{~N}$ & $64-79$ & $20-32$ & $0.004-0.007$ & $0.9-1.3$ & - \\
\hline & & & & $\mathrm{N}$ & $63-82$ & $18-34$ & $0.03-0.1$ & $0.7-1.0$ & - \\
\hline & & & & $\mathrm{Y}$ & $61-82$ & $13-32$ & $0.004-0.007$ & $0.9-1.3$ & $0-11$ \\
\hline & & & & $\mathrm{Y}$ & $33-85$ & $11-57$ & $0.03-1.4$ & $0.3-1.1$ & $0-17$ \\
\hline \multirow{2}{*}{ J0218-00 } & $\mathrm{N}$ & $\mathrm{Y}$ & 17 & $\mathrm{~N}$ & $13-39$ & $60-87$ & $0.03-0.09$ & $0.0-0.3$ & - \\
\hline & & & & $\mathrm{Y}$ & $0-37$ & $43-99$ & $0.03-0.2$ & $0.0-0.5$ & $0-10$ \\
\hline J0218-00 & $\mathrm{E}$ & & 17 & $\mathrm{~N}$ & $10-33$ & $66-81$ & $0.02-0.04$ & $0.1-0.4$ & - \\
\hline \multirow[t]{2}{*}{$\mathrm{J} 0227+01$} & $\mathrm{~N}$ & $\mathrm{Y}$ & 11 & $\mathrm{~N}$ & $15-52$ & $46-82$ & $0.03-0.1$ & $0.1-0.5$ & - \\
\hline & & & & $\mathrm{Y}$ & $6-49$ & $32-89$ & $0.03-0.3$ & $0.1-0.6$ & $0-10$ \\
\hline \multirow[t]{2}{*}{ J0234-07 } & $\mathrm{N}$ & $\mathrm{N}$ & 25 & $\mathrm{~N}$ & $41-85$ & $15-55$ & $0.001-0.02$ & $0-0.7$ & - \\
\hline & & & & $\mathrm{Y}$ & $0-82$ & 0-90 & $0.001-5$ & $0-2$ & $0-33$ \\
\hline \multirow{2}{*}{$\mathrm{J} 0249+00$} & $\mathrm{~N}$ & $\mathrm{~N}$ & 25 & $\mathrm{~N}$ & $12-35$ & $65-88$ & $0.05-0.1$ & $0.7-0.8$ & - \\
\hline & & & & $\mathrm{Y}$ & $0-41$ & $47-89$ & $0.05-0.7$ & $0.4-0.8$ & $0-30$ \\
\hline \multirow[t]{5}{*}{$\mathrm{J} 0320+00$} & $\mathrm{~N}$ & $\mathrm{Y}$ & 8 & $\mathrm{~N}$ & $37-47$ & $50-61$ & $0.005-0.006$ & $0.1-0.3$ & - \\
\hline & & & & $\mathrm{N}$ & $33-46$ & $52-64$ & $0.02-0.03$ & $0.0-0.2$ & - \\
\hline & & & & $\mathrm{Y}$ & $18-25$ & $69-77$ & 0.001 & 0.7 & $<1$ \\
\hline & & & & $\mathrm{Y}$ & $12-47$ & $35-83$ & $0.004-0.006$ & $0.1-0.6$ & $0-15$ \\
\hline & & & & $\mathrm{Y}$ & $6-48$ & $19-87$ & $0.02-0.2$ & $0-0.5$ & $0-15$ \\
\hline J0332-00 & $\mathrm{N}$ & $\mathrm{N}$ & 4 & $\mathrm{Y}$ & $0-76$ & $0-51$ & $0.001-5$ & $0-2$ & $0-47$ \\
\hline \multirow[t]{5}{*}{ J0332-00 } & $\mathrm{E}$ & & 3 & $\mathrm{~N}$ & $64-70$ & $25-29$ & $0.001-0.002$ & 0.8 & - \\
\hline & & & & $\mathrm{N}$ & $59-75$ & $22-35$ & $0.004-0.006$ & $0.4-0.8$ & - \\
\hline & & & & $\mathrm{N}$ & $65-75$ & $23-31$ & $0.03-0.05$ & $0.2-0.4$ & - \\
\hline & & & & $\mathrm{Y}$ & $57-76$ & $11-36$ & $0.004-0.007$ & $0.3-0.8$ & $0-18$ \\
\hline & & & & $\mathrm{Y}$ & $0-78$ & $8-85$ & $0.03-4$ & $0-0.6$ & $0-25$ \\
\hline \multirow{2}{*}{$\mathrm{J} 0334+00$} & $\mathrm{~N}$ & $\mathrm{Y}$ & 23 & $\mathrm{~N}$ & $6-27$ & 74-97 & 0.04-0.09 & $0-0.2$ & - \\
\hline & & & & $\mathrm{Y}$ & $0-27$ & $55-104$ & $0.04-0.2$ & $0-0.3$ & $0-10$ \\
\hline \multirow[t]{2}{*}{$\mathrm{J} 0848+01$} & $\mathrm{~N}$ & $\mathrm{Y}$ & 19 & $\mathrm{~N}$ & $53-66$ & $32-43$ & $0.2-0.4$ & $0.1-0.3$ & - \\
\hline & & & & $\mathrm{Y}$ & $31-65$ & $31-55$ & $0.3-0.5$ & $0.1-0.3$ & $0-15$ \\
\hline \multirow[t]{3}{*}{ J0904-00 } & $\mathrm{N}$ & $\mathrm{Y}$ & 13 & $\mathrm{~N}$ & $12-32$ & $72-91$ & 0.006 & $0.3-04$ & - \\
\hline & & & & $\mathrm{N}$ & $11-31$ & $73-92$ & 0.03 & $0.2-0.3$ & - \\
\hline & & & & $\mathrm{Y}$ & $0-35$ & $51-99$ & $0.03-0.04$ & $0.1-0.4$ & $0-10$ \\
\hline J0923+01 & $\mathrm{N}$ & $\mathrm{Y}$ & 14 & $\mathrm{~N}$ & $0-19$ & $80-100$ & $0.05-0.1$ & $0.4-0.5$ & - \\
\hline & & & & $\mathrm{Y}$ & $0-22$ & $55-100$ & $0.05-0.2$ & $0.1-0.6$ & $0-20$ \\
\hline J0924+01 & $\mathrm{N}$ & $\mathrm{N}$ & 14 & $\mathrm{~N}$ & $10-20$ & $77-86$ & 0.005 & 1 & - \\
\hline & & & & $\mathrm{N}$ & $19-28$ & $71-78$ & 0.02 & 0.8 & - \\
\hline & & & & $\mathrm{Y}$ & $6-44$ & $27-88$ & $0.004-0.007$ & $0.4-1.1$ & $0-44$ \\
\hline & & & & $\mathrm{Y}$ & $0-46$ & $16-86$ & $0.02-1.2$ & $0-1.1$ & $0-60$ \\
\hline
\end{tabular}


Table 5 - continued.

\begin{tabular}{|c|c|c|c|c|c|c|c|c|c|}
\hline Name & Aperture & $\begin{array}{l}\text { Balmer } \\
\text { lines? }\end{array}$ & $\begin{array}{l}\text { Nebular } \\
\text { (per cent) }\end{array}$ & PL? & $\begin{array}{c}\text { OSP } \\
\text { (per cent) }\end{array}$ & $\begin{array}{c}\text { YSP } \\
\text { (per cent) }\end{array}$ & $\begin{array}{l}\text { YSP age } \\
\text { (Gyr) }\end{array}$ & $E(B-V)$ & $\begin{array}{r}\text { Power law } \\
\text { (per cent) }\end{array}$ \\
\hline \multirow[t]{2}{*}{$\mathrm{J} 0948+00$} & \multirow[t]{2}{*}{$\mathrm{N}$} & \multirow[t]{2}{*}{$Y$} & \multirow[t]{2}{*}{15} & $\mathrm{~N}$ & $30-46$ & $53-68$ & $0.2-0.3$ & $0-0.2$ & - \\
\hline & & & & Y & $0-47$ & $51-94$ & $0.2-0.4$ & $0.1-0.5$ & $0-20$ \\
\hline \multirow[t]{5}{*}{$\mathrm{J} 1307-02$} & \multirow[t]{5}{*}{$\mathrm{N}$} & \multirow[t]{5}{*}{$\mathrm{N}$} & \multirow[t]{5}{*}{27} & $\mathrm{~N}$ & - & - & - & - & - \\
\hline & & & & $\mathrm{Y}$ & $36-58$ & $24-46$ & 0.007 & $0.2-0.3$ & $8-25$ \\
\hline & & & & $\mathrm{Y}$ & $20-46$ & $39-71$ & $0.006-0.007$ & $0.8-0.9$ & $1-23$ \\
\hline & & & & $\mathrm{Y}$ & $35-61$ & $21-51$ & $0.03-0.1$ & $0-0.4$ & $4-25$ \\
\hline & & & & $\mathrm{Y}$ & $0-47$ & $33-88$ & $0.02-1.0$ & $0.4-0.9$ & $1-25$ \\
\hline \multirow[t]{2}{*}{$\mathrm{J} 1337-01$} & \multirow[t]{2}{*}{$\mathrm{N}$} & \multirow[t]{2}{*}{ Y } & \multirow[t]{2}{*}{8} & $\mathrm{~N}$ & $0-30$ & 74-100 & $0.04-0.1$ & $0.3-0.5$ & - \\
\hline & & & & Y & $0-39$ & $55-100$ & $0.04-0.1$ & $0.1-0.5$ & $1-15$ \\
\hline
\end{tabular}
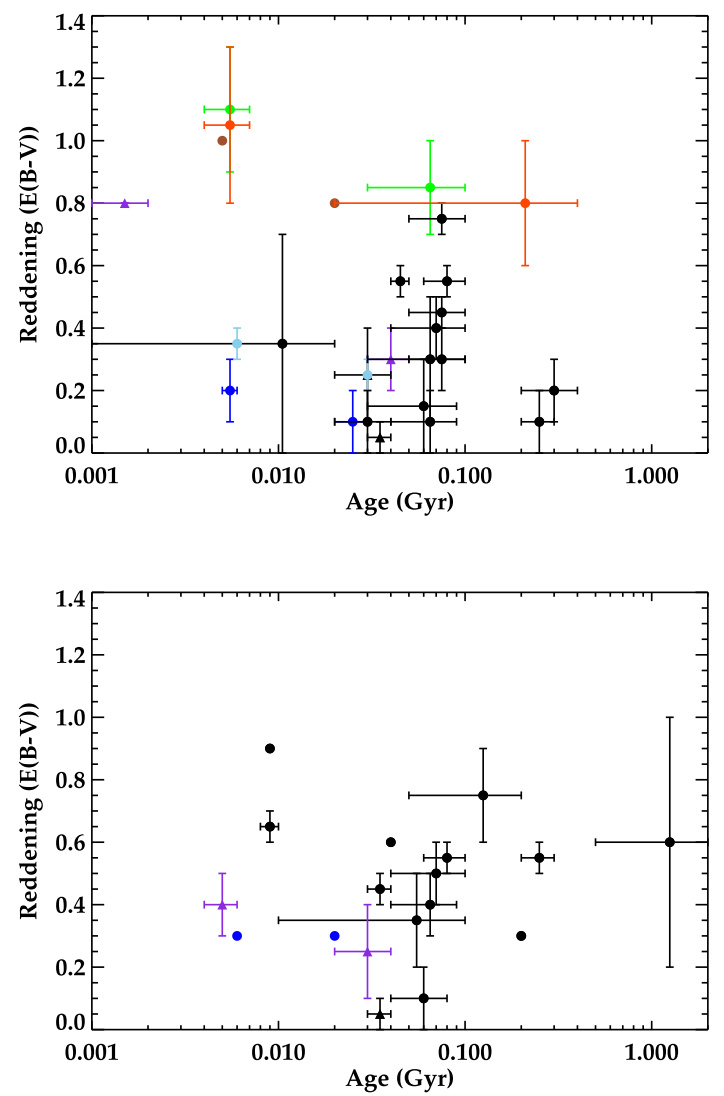

Figure 6. The mean age and reddening found for each of the spectroscopic apertures. The top panel shows the results for combination 2 and the bottom panel shows the results for combination 3 . In both panels, the mean reddening of the YSP is plotted against the mean age found for each aperture. In the cases where, for an individual object, the modelling produced more than one region of acceptable fits with $\chi_{\text {red }}^{2}<1$, both regions are plotted in the same colour (i.e. they refer to the same object), otherwise, the result for each object is plotted in black. The results related to apertures extract from the nuclear regions are plotted as circles, whilst those extract from off-nuclear regions a plotted as triangles. The bars show the range of values of both age and reddening that provide acceptable fits.

of active and merging systems. Are the ages found here typical of those found in other studies?

In Bessiere et al. (2012), the 2 Jy sample ${ }^{6}$ (Tadhunter et al. 1993, 1998) was introduced, in order to compare the optical

${ }^{6}$ Full details of the 2Jy sample and available data can be found at http://2jy.extragalactic.info/Home.html. morphologies of PRG with the type II quasars presented here. To recap the selection criteria, the 2 Jy objects have radio fluxes $S_{2.7 \mathrm{GHz}}>2.0 \mathrm{Jy}$, radio spectral indices $\alpha_{2.7}^{4.8}>0.5\left(F_{v} \propto v^{-\alpha}\right)$, declinations $\delta<+10^{\circ}$ and redshifts $0.05<z<0.7$. For further details on the criteria used for selection and completeness, refer to Tadhunter et al. (1993, 1998). The same stellar synthesis modelling technique has been applied to this sample of PRG facilitating a direct comparison of the incidence of YSPs in two samples.

Tadhunter et al. (2002) present results for an RA limited subsample of that discussed in Bessiere et al. (2012), comprising 22 PRG $(0.15<z<0.7)$. Their modelling showed that only $\sim 15$ per cent of these PRG harbour a clearly detectable, significant YSP. These findings were based on both stellar synthesis modelling and the clear detection of Balmer absorption features in the spectra. The remaining 19 objects were adequately fit by an elliptical galaxy template combined with a power-law component (i.e. combination 1) contributing between 20 and 90 per cent of the continuum flux just below the $4000 \AA$ break.

These results have been further updated (Holt et al. 2006, 2007; Wills et al. 2008) with the availability of deeper data with improved $\mathrm{S} / \mathrm{N}$ in the continuum, higher spectral resolution and, in some cases, wider spectral coverage, combined with the use of higher resolution stellar population models (Bruzual \& Charlot 2003). This allowed firm detections of YSPs in a further two objects from the original sample, while improving the age estimates for three sources presented in Tadhunter et al. (2002). This brings the detection rate of YSPs in this subset of the 2 Jy sample to $5 / 22$ (23 per cent), with $4 / 5$ of these having $t_{\max } \leq 100 \mathrm{Myr}$. Therefore, the ages of the YSPs, when detected, are consistent with those found for the type II quasars (Fig. 7), although the detection rate is significantly lower. This detection rate is in stark contrast to the results presented here for the type II quasars, where only two object (J0234-07 and J0332-00) can be adequately fitted with an OSP + power-law combination of components alone.

Taking into consideration the results of the comparison of optical morphologies and photometric properties presented in Bessiere et al. (2012), this result could be considered somewhat unexpected. This is because the absolute magnitudes, rate of interactions, distribution of host galaxies amongst the morphological groups and the distribution of the surface brightnesses of the tidal features associated with the mergers were shown to be similar for the two samples, suggesting that both types of AGN reside in similar host galaxies. In contrast, the significant differences in the stellar content of their host galaxies suggests that they are fundamentally different systems.

Although PRG are almost invariably massive ellipticals, the case is not quite so clear cut for radio-quiet quasars. If they are more 
Table 6. The results of our stellar synthesis modelling assuming an $2 \mathrm{Gyr}$ underlying stellar population. Columns 1 and 2 give the object name and aperture (N: nuclear; E: Extended; G: companion galaxy). Column 3 shows whether the higher order Balmer absorption lines were detected in the unsubtracted spectrum. Column 4 gives the nebular continuum contribution in the wavelength range 3540-3640 , whilst column 5 indicates whether a power-law component was included in the model. Columns 6 and 7 show the range of percentage contributions to the total flux of both the ISP and YSP, whilst columns 7 and 8 show the range of ages and reddenings that are acceptable for the YSPs. Finally, column 8 gives the range of percentage contribution by a power-law component.

\begin{tabular}{|c|c|c|c|c|c|c|c|c|c|}
\hline Name & Aperture & $\begin{array}{l}\text { Balmer } \\
\text { lines? }\end{array}$ & $\begin{array}{l}\text { Nebular } \\
\text { (per cent) }\end{array}$ & PL? & $\begin{array}{c}\text { ISP } \\
\text { (per cent) }\end{array}$ & $\begin{array}{c}\text { YSP } \\
\text { (per cent) }\end{array}$ & $\begin{array}{l}\text { YSP age } \\
\text { (Gyr) }\end{array}$ & $E(B-V)$ & $\begin{array}{c}\text { Power law } \\
\text { (per cent) }\end{array}$ \\
\hline \multirow{2}{*}{$\mathrm{J} 2358-00$} & $\mathrm{~N}$ & $\mathrm{Y}$ & 28 & $\mathrm{~N}$ & $0-13$ & 89-100 & 0.04 & 0.6 & - \\
\hline & & & & Y & 20-91 & 54-104 & $0.04-0.2$ & $0.4-0.7$ & $0-29$ \\
\hline \multirow[t]{2}{*}{ J0025-10 } & $\mathrm{N}$ & $\mathrm{Y}$ & 3 & $\mathrm{~N}$ & - & - & - & - & - \\
\hline & & & & $\mathrm{Y}$ & $0-42$ & 39-94 & $0.02-0.4$ & $0-0.4$ & $0-42$ \\
\hline J0025-10 & $\mathrm{G}$ & $\mathrm{Y}$ & 10 & $\mathrm{~N}$ & - & - & - & - & - \\
\hline J0025-10 & $\mathrm{E}$ & $\mathrm{Y}$ & 9 & $\mathrm{~N}$ & $6-24$ & 73-91 & $0.03-0.04$ & $0-0.1$ & - \\
\hline \multirow[t]{2}{*}{$\mathrm{J} 0114+00$} & $\mathrm{~N}$ & $\mathrm{~N}$ & 14 & $\mathrm{~N}$ & $0-71$ & 30-98 & $0.5-2$ & $0.2-1.0$ & - \\
\hline & & & & $\mathrm{Y}$ & $0-71$ & $28-100$ & $0.5-2$ & $0.2-1.0$ & $0-9$ \\
\hline \multirow[t]{2}{*}{$\mathrm{J} 0123+00$} & $\mathrm{~N}$ & $\mathrm{~N}$ & 20 & $\mathrm{~N}$ & - & - & - & - & - \\
\hline & & & & $\mathrm{Y}$ & - & - & - & - & - \\
\hline \multirow{2}{*}{$\mathrm{J} 0142+14$} & $\mathrm{~N}$ & $\mathrm{Y}$ & - & $\mathrm{N}$ & $0-33$ & $70-100$ & $0.04-0.1$ & $0.2-0.5$ & - \\
\hline & & & & $\mathrm{Y}$ & $0-42$ & $62-104$ & $0.04-0.1$ & $0.2-0.5$ & $0-45$ \\
\hline \multirow[t]{4}{*}{ J0217-00 } & $\mathrm{N}$ & $\mathrm{Y}$ & 14 & $\mathrm{~N}$ & $0-37$ & $65-100$ & $0.06-0.1$ & $0.5-0.6$ & - \\
\hline & & & & $\mathrm{Y}$ & $0-40$ & $52-100$ & $0.06-0.3$ & $0.2-0.6$ & $0-15$ \\
\hline & GTC & & & $\mathrm{N}$ & $0-38$ & 65-99 & $0.04-0.1$ & $0.5-0.6$ & - \\
\hline & & & & $\mathrm{Y}$ & $0-53$ & 47-99 & $0.04-0.3$ & $0.2-0.7$ & $0-15$ \\
\hline \multirow{2}{*}{ J0217-01 } & $\mathrm{N}$ & $\mathrm{N}$ & 20 & $\mathrm{~N}$ & - & - & - & - & - \\
\hline & & & & $\mathrm{Y}$ & - & - & - & - & - \\
\hline \multirow[t]{2}{*}{ J0218-00 } & $\mathrm{N}$ & $\mathrm{Y}$ & 17 & $\mathrm{~N}$ & - & - & - & - & - \\
\hline & & & & $\mathrm{Y}$ & $0-41$ & 45-99 & $0.04-0.2$ & $0.1-0.4$ & $0-10$ \\
\hline \multirow[t]{2}{*}{ J0218-00 } & $\mathrm{E}$ & & 17 & $\mathrm{~N}$ & $24-41$ & $56-74$ & $0.004-0.006$ & $0.3-0.5$ & - \\
\hline & & & & $\mathrm{N}$ & $15-44$ & $56-81$ & $0.02-0.04$ & $0.1-0.4$ & - \\
\hline \multirow[t]{2}{*}{$\mathrm{J} 0227+01$} & $\mathrm{~N}$ & $\mathrm{Y}$ & 11 & $\mathrm{~N}$ & $26-52$ & $46-72$ & $0.03-0.04$ & $0.4-0.5$ & - \\
\hline & & & & $\mathrm{Y}$ & $12-71$ & $25-80$ & $0.03-0.2$ & $0.0-0.7$ & $0-10$ \\
\hline \multirow[t]{4}{*}{ J0234-07 } & $\mathrm{N}$ & $\mathrm{N}$ & 25 & $\mathrm{~N}$ & - & - & - & - & - \\
\hline & & & & $\mathrm{Y}$ & $68-88$ & $6-11$ & $0.001-0.003$ & $0-0.1$ & $3-20$ \\
\hline & & & & $\mathrm{Y}$ & 59-91 & $4-34$ & $0.001-0.3$ & $0.7-1.6$ & $1-14$ \\
\hline & & & & $\mathrm{Y}$ & $0-83$ & $9-83$ & $1.2-2$ & $0.2-0.5$ & $6-20$ \\
\hline \multirow[t]{2}{*}{$\mathrm{J} 0249+00$} & $\mathrm{~N}$ & $\mathrm{~N}$ & 25 & $\mathrm{~N}$ & $19-59$ & $44-81$ & $0.05-0.2$ & $0.6-0.9$ & - \\
\hline & & & & $\mathrm{Y}$ & $1-61$ & $41-84$ & $0.06-0.6$ & $0.5-0.8$ & $0-26$ \\
\hline \multirow{3}{*}{$\mathrm{J} 0320+00$} & $\mathrm{~N}$ & $\mathrm{Y}$ & 8 & $\mathrm{~N}$ & - & - & - & - & - \\
\hline & & & & $\mathrm{Y}$ & $10-49$ & $27-82$ & $0.001-0.006$ & $0.6-0.8$ & $0-15$ \\
\hline & & & & $\mathrm{Y}$ & $16-55$ & $25-76$ & $0.009-0.1$ & $0.3-0.6$ & $0-15$ \\
\hline \multirow[t]{3}{*}{$\mathrm{J} 0332-00$} & $\mathrm{~N}$ & $\mathrm{~N}$ & 4 & $\mathrm{Y}$ & $46-71$ & $19-28$ & $0.001-0.003$ & $0-0.1$ & $3-27$ \\
\hline & & & & $\mathrm{Y}$ & $40-73$ & $14-47$ & $0.001-0.004$ & $1-1.3$ & $5-20$ \\
\hline & & & & $\mathrm{Y}$ & $26-73$ & $13-63$ & $0.008-0.1$ & $0.5-0.9$ & $4-22$ \\
\hline J0332-00 & $\mathrm{E}$ & & 3 & $\mathrm{~N}$ & - & - & - & - & - \\
\hline & & & & $\mathrm{Y}$ & - & - & - & - & - \\
\hline $\mathrm{J} 0334+00$ & $\mathrm{~N}$ & $\mathrm{Y}$ & 23 & $\mathrm{~N}$ & $9-32$ & $71-95$ & $0.04-0.08$ & $0-0.2$ & - \\
\hline & & & & $\mathrm{Y}$ & 0-39 & $49-100$ & $0.04-0.2$ & $0-0.4$ & $0-10$ \\
\hline J0848+01 & $\mathrm{N}$ & $\mathrm{Y}$ & 19 & $\mathrm{~N}$ & $56-72$ & $23-38$ & $0.2-0.3$ & $0.5-0.6$ & - \\
\hline & & & & $\mathrm{Y}$ & $56-79$ & $21-42$ & 0.3 & 0.5 & $0-8$ \\
\hline J0904-00 & $\mathrm{N}$ & $\mathrm{Y}$ & 13 & $\mathrm{~N}$ & $16-22$ & $82-87$ & 0.006 & 0.4 & - \\
\hline & & & & $\mathrm{N}$ & $26-31$ & $72-76$ & 0.02 & 0.3 & - \\
\hline & & & & $\mathrm{Y}$ & $0-42$ & 49-98 & $0.02-0.04$ & $0.2-0.4$ & $0-10$ \\
\hline J0923+01 & $\mathrm{N}$ & $\mathrm{Y}$ & 14 & $\mathrm{~N}$ & $0-26$ & $74-100$ & $0.04-0.1$ & $0.4-0.6$ & - \\
\hline & & & & Y & $0-33$ & $48-100$ & $0.05-0.2$ & $0.1-0.6$ & $0-20$ \\
\hline J0924+01 & $\mathrm{N}$ & $\mathrm{N}$ & 14 & $\mathrm{~N}$ & $36-51$ & $46-59$ & 0.009 & 0.9 & - \\
\hline & & & & $\mathrm{Y}$ & $13-68$ & $4-81$ & $0.001-0.007$ & $0-1.5$ & $0-45$ \\
\hline & & & & $\mathrm{Y}$ & $0-67$ & $5-88$ & $0.009-1.2$ & $0-1.4$ & $0-60$ \\
\hline $\mathrm{J} 0948+00$ & $\mathrm{~N}$ & $\mathrm{Y}$ & 15 & $\mathrm{~N}$ & $30-40$ & $58-69$ & 0.2 & 0.3 & - \\
\hline & & & & $\mathrm{Y}$ & $0-57$ & $41-94$ & $0.2-0.4$ & $0.1-0.4$ & $0-19$ \\
\hline $\mathrm{J} 1307-02$ & $\mathrm{~N}$ & $\mathrm{~N}$ & 27 & $\mathrm{~N}$ & $37-54$ & $47-62$ & $0.008-0.01$ & $0.6-0.7$ & - \\
\hline & & & & Y & $44-74$ & $25-50$ & $0.001-0.002$ & $1.2-1.3$ & $2-8$ \\
\hline & & & & $\mathrm{Y}$ & $29-76$ & $19-66$ & $0.004-0.007$ & $0.9-1.2$ & $2-23$ \\
\hline & & & & $\mathrm{Y}$ & $53-80$ & $9-25$ & $0.03-0.05$ & $0-0.2$ & $8-25$ \\
\hline & & & & $\mathrm{Y}$ & $0-79$ & $14-88$ & $0.009-1.2$ & $0.4-1.2$ & $1-25$ \\
\hline $\mathrm{J} 1337-01$ & $\mathrm{~N}$ & $\mathrm{Y}$ & 8 & $\mathrm{~N}$ & $0-40$ & $66-100$ & $0.04-0.09$ & $0.3-0.5$ & - \\
\hline & & & & $\mathrm{Y}$ & $0-47$ & $46-100$ & $0.04-0.2$ & $0-0.5$ & $0-15$ \\
\hline
\end{tabular}



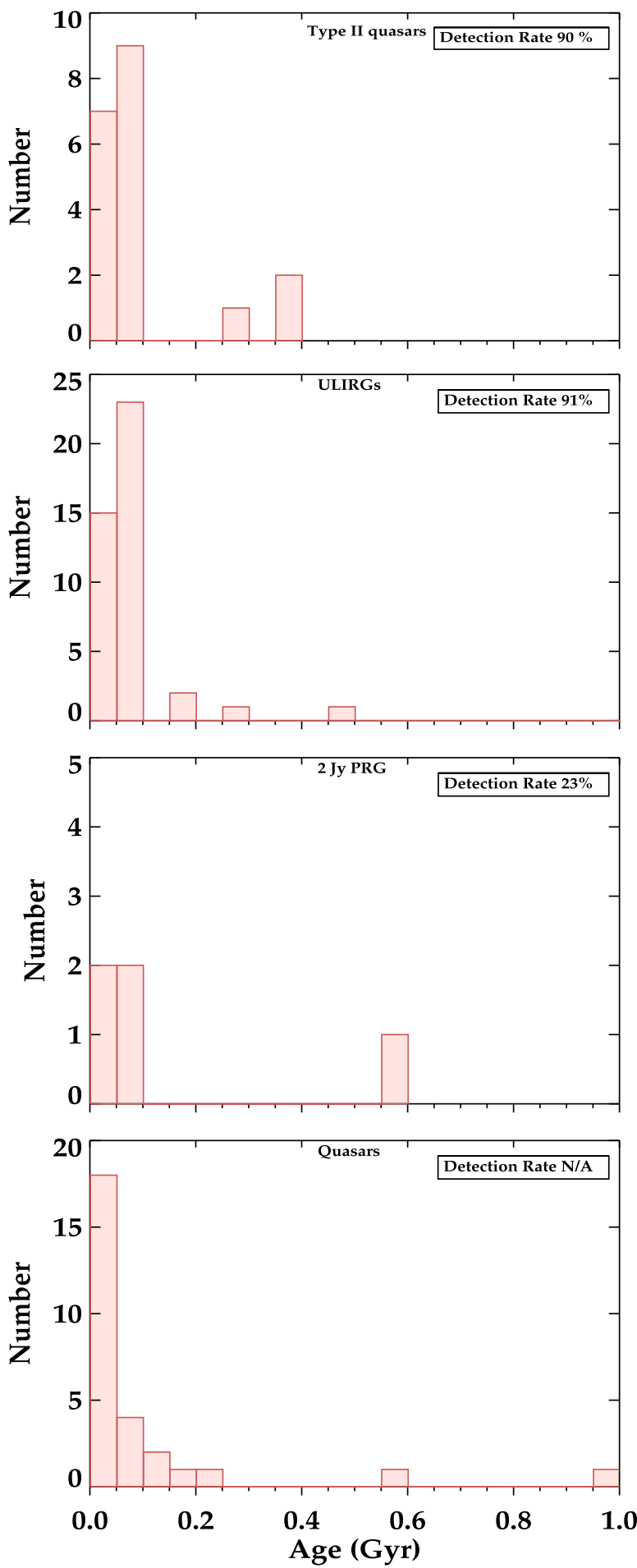

Figure 7. Comparison of the YSP ages found for the different samples discussed in this work. The top panel shows the distribution of ages for the type II quasars investigated in this paper. The following two histograms show the distribution of ages for the ULIRGs and 2 Jy PRGs, respectively. The final panel shows the distribution of ages for a compilation of samples taken from the literature and includes results from Heckman et al. (1997), Canalizo \& Stockton (2000), Tadhunter et al. (2005, 2011), Emonts et al. (2006), Holt et al. (2006, 2007), Wills et al. (2008), Liu et al. (2009) and Villar-Martín et al. (2012). The objects shown in this panel include only AGN that have quasar-like luminosities $\left.\left(L_{[\mathrm{O}} \mathrm{III}\right]>10^{35} \mathrm{~W}\right)$. In each of the panels in which 'complete' samples have been considered, the detection rate of YSPs in the host galaxies is also given. likely to have a significant disc component than their radio-loud counterparts (Floyd et al. 2004), this could mean that we would naturally expect star formation to be more prevalent. However, the deep $r^{\prime}$-band imaging presented in Bessiere et al. (2012) strongly suggests that the majority of these objects are not disc dominated, a finding supported by Wylezalek et al. (2016), who also find that the majority of their sample (90 per cent) of 20 type II quasars (including $\mathrm{J} 0123+00$ and $\mathrm{J} 2358-00$ ) are bulge dominated. This suggests that differences in galaxy morphology are unlikely to be the prime origin of the differences in stellar content. Wylezalek et al. (2016) also derive stellar masses for their sample and find an average of $\log \left(M_{*} / \mathrm{M}_{\odot}\right)=10.7 \pm 0.3$, consistent with those found for our sample of type II quasars $\left(\sim 10^{11}\right.$; Bessiere 2014$)$, and below that found for PRG with similar stellar populations. This may imply that stellar mass is the more fundamental difference between PRG and radio-quiet type II quasars. The comparison between the stellar masses of the two types of objects will be discussed in more detail in a forthcoming paper.

However, it is important to recognize that the quasar luminosity in a number of these PRG is significantly higher than those of many of the type II quasars presented here. The highest [O III] luminosity of a type II quasar in our sample is $\log \left(L_{[\mathrm{OIII}]} / \mathrm{L}_{\odot}\right)=9.32$, whilst 6 of the 22 PRG have a higher $L_{[\mathrm{O} I I I]}$. This will have the effect of making it more difficult to detect signatures of YSPs in the host galaxies because of the stronger AGN-related continuum components, such as scattered quasar light and nebular continuum. It will also be more difficult to detect the Balmer lines in absorption because there will be a higher degree of contamination by Balmer emission lines. Therefore, the detection rate of 23 per cent must be taken as a lower limit to the proportion of PRG that host YSPs.

\subsection{Comparison with ULIRGs}

In the context of evolutionary scenarios, such as that proposed by Sanders et al. (1988), it is worthwhile to compare the results derived here with those found for a complete sample of ULIRGs presented by Rodríguez Zaurín et al. (2009) and Rodríguez Zaurín, Tadhunter \& González Delgado (2010). This sample consists of 36 ULIRGs, of which 14 have been classified as Seyferts. The full sample includes a complete RA and Dec limited sample of 26 objects with redshifts $z<0.13$, and in addition, a further 10 objects $(z<0.18)$ were included because they are characterized by warm infrared colours. Again, the same stellar synthesis modelling technique as that employed here was used.

Rodríguez Zaurín et al. (2009) extracted 5 kpc nuclear apertures for all the objects in their sample, as well as apertures from extended tidal features. Due to the fact that in all but three cases, only one nuclear aperture was extracted from each type II quasar, we will only consider apertures centred on the nucleus, thus allowing a more meaningful comparison.

Their modelling also utilized a combination of an OSP and YSP, although they use an OSP with $t_{\mathrm{OSP}}=12.5 \mathrm{Gyr}$. This use of an older underlying population is unlikely to have a significant effect on the YSP ages because the differences between the stellar templates at large ages is minimal.

The results presented for the ULIRGs in Rodríguez Zaurín et al. (2010) are very similar in terms of the YSP ages found for the nuclear apertures, the reddenings associated with the YSPs and the contribution made by the YSP to the total flux in the normalizing bin. They find that 91 per cent of the nuclear apertures have 


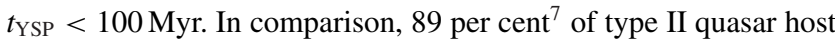
galaxies that require a YSP component, also have $t_{\mathrm{YSP}}<100 \mathrm{Myr}$ for the same modelling combination. Fig. 7 shows that the distributions of ages of the type II quasars and ULIRGs are very similar, with similar detection rates. In terms of the reddenings found for the YSPs, Rodríguez Zaurín et al. (2010) derived a median value of $E(B-V)=0.4$ for the apertures extracted from the nuclear regions of the ULIRGs. This is the same as the median value found for this sample of type II quasars, with $E(B-V)=0.4$.

\section{TIMING THE QUASAR ACTIVITY IN GALAXY MERGERS.}

Based on the above results, it is clear that in the majority of cases when no power-law component is included, a YSP with $t_{\max }<100 \mathrm{Myr}$ is a significant flux component of the quasar host galaxies studied here: for combination $2 \sim 89$ per cent have a significant YSP with $t_{\max } \leq 100 \mathrm{Myr}$, while $\sim 32$ per cent have a significant YSP with $t_{\max } \leq 50 \mathrm{Myr}$.

It is interesting to consider these results in the context of an evolutionary sequence such as that of Sanders et al. (1988). In such a sequence, the merger will initiate a substantial burst of star formation, leading to the system being classified as a (U)LIRG, which will then evolve into an observable quasar. These kinds of evolutionary models (e.g. di Matteo, Springel \& Hernquist 2005; Springel, Di Matteo \& Hernquist 2005; Hopkins et al. 2006) predict that, directly following the merger, we will observe a system with a YSP that has been heavily reddened by the enshrouding dust. At this stage of the sequence, the quasar activity will be too heavily obscured to be detectable in the optical. As the merger progresses, feedback processes (usually attributed to the AGN activity) will shut down or quench star formation activity, while at the same time removing the material enshrouding the quasar. The observable result would be a single nucleus and an ageing (post) starburst population. If this scenario is correct, then we should observe a delay between the merger driven starburst and the visible quasar activity.

Using various stellar population modelling techniques, some previous studies of AGN host galaxies have found evidence of just such a delay, ranging from a few 100 Myr to a few Gyr (Cid Fernandes et al. 2004; Tadhunter et al. 2005, 2011; Emonts et al. 2006; Davies et al. 2007; Holt et al. 2007; Wills et al. 2008; Wild et al. 2010; Canalizo \& Stockton 2013; Ramos Almeida et al. 2013). This is also true of two of the type II quasar objects presented here, in which delays of up to a few $100 \mathrm{Myr}$ (assuming combination 2) are found. However, other studies have shown that no such delay exists and that the quasar and starburst activity are triggered quasi-simultaneously (Heckman et al. 1997; Canalizo \& Stockton 2000, 2001; Brotherton et al. 2002; Holt et al. 2007; Wills et al. 2008; Liu et al. 2009; Tadhunter et al. 2011; Villar-Martín et al. 2012; Bessiere et al. 2014).

We must then consider the causes of these apparently ambiguous results. One explanation may be that the age of the stellar population hosted by an AGN is dependent on the luminosity of the AGN itself. Evidence to support this explanation can be found by examining the results presented for the 2 Jy sample of PRG (Tadhunter et al. 2011), which show that host galaxies with YSPs with ages $t_{\mathrm{YSP}}<0.1 \mathrm{Gyr}$ also tend to host AGN of quasar-like luminosity $\left(\log \left(L_{[\mathrm{O} I \mathrm{II}]} / \mathrm{L}_{\odot}\right)>8.5\right)$. In contrast, when we consider those objects in the sample that fall below this luminosity threshold,

\footnotetext{
${ }^{7}$ This proportion excludes $\mathrm{J} 0123+00$ and J0332-00, which cannot be modelled without the inclusion of a power-law component.
}

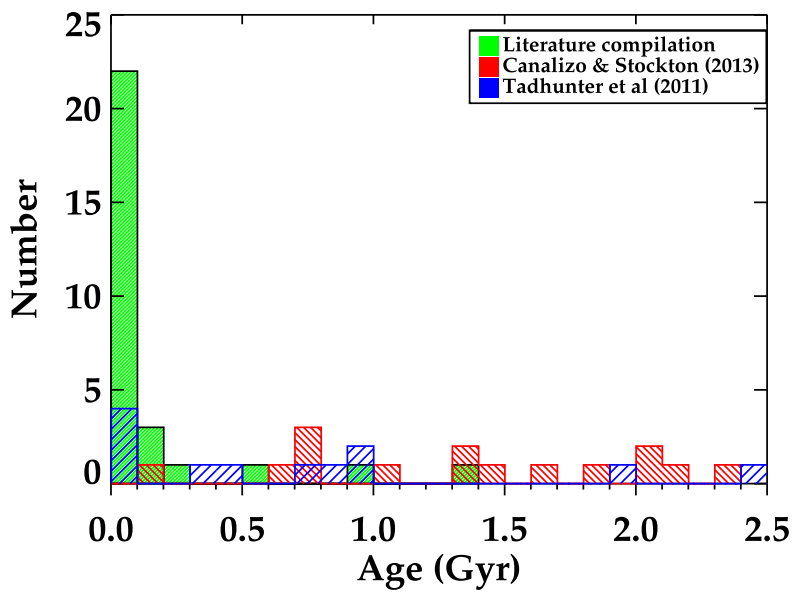

Figure 8. The same samples of quasars as shown in the final panel of Fig. 7 (green) but also including the lower luminosity AGN discussed by Tadhunter et al. (2011) $\left(L_{[\mathrm{O} I I]}<10^{35} \mathrm{~W}\right.$; blue) and also the sample of quasars presented in Canalizo \& Stockton (2013, red).

as well as including the results of other studies dominated by lower luminosity objects (Emonts et al. 2005; Tadhunter et al. 2005; Wild et al. 2010), YSP ages $0.2<t_{\mathrm{YSP}}<2$ Gyr are more commonly found. This is effectively demonstrated in Fig. 7, where the distribution of YSP ages is shown for various samples of quasars (see the caption for details), and Fig. 8 that shows the same samples but with the inclusion of lower luminosity PRG (as well as the sample of Canalizo \& Stockton 2013).

Taking this luminosity dependence into consideration, if we concentrate on the quasar-like objects with the best spectroscopic data on the stellar populations (Canalizo \& Stockton 2000; Holt et al. 2007; Wills et al. 2008; Tadhunter et al. 2011; Villar-Martín et al. 2012; Canalizo \& Stockton 2013; Bessiere et al. 2014), in general, ages $t_{\mathrm{YSP}}<0.1 \mathrm{Gyr}$ are typical in the nuclear regions. Examples of such cases include the PRG 3C459 (Wills et al. 2008), the type II quasar J0025-10 (Bessiere et al. 2014) and the nearby quasar Mrk 231 (Canalizo \& Stockton 2001; Rodríguez Zaurín et al. 2009) in which the populations are found to have ages $t_{\mathrm{YSP}}<0.1 \mathrm{Gyr}$. In the latter case, the findings are supported by the detection of the He I absorption features associated with B-type stars (González Delgado, Leitherer \& Heckman 1999). The nearby type II quasar Mrk 447 (Heckman et al. 1997) is clearly identified as having a YSP with an age of $t_{\text {YSP }} \sim 6 \mathrm{Myr}$, supported by the presence of UV absorption features, such as $\mathrm{Si}$ III $\lambda 1417$, which are attributed to the presence of late $\mathrm{O}$ and early B supergiants. Fig. 7 shows how the results of these various studies compare with those found in this work.

Despite using high-quality spectra, one notable exception to this trend is the study of Canalizo \& Stockton (2013), who find that, although 14/15 quasar host galaxies do have evidence for a significant YSP, they are all older $\left(t_{\mathrm{ISP}} \sim 1.2 \mathrm{Gyr}\right)$ than those found in the majority of other quasar-like systems. Clearly, it is important to understand the root of this discrepancy. One possible explanation is that Canalizo \& Stockton do not directly consider reddening of the YSP in their analysis. The importance of accounting for reddening has been underscored by spectroscopic studies of the YSPs of local radio-galaxies and ULIRGs (Tadhunter et al. 2005; Rodríguez Zaurín et al. 2009, 2010). If reddening of the YSP is not adequately accommodated in the modelling, a YSP can appear substantially older than it is in reality. However, based on their fits to the stellar absorption line features, they conclude that any YSP/ISP present cannot be significantly younger than that their primary modelling 
suggests. They also perform a double check on their fits, which includes a low-order multiplicative polynomial over a spectral window that includes the Balmer absorption features, which can account for reddening (for full details, refer to Canalizo \& Stockton 2013), and find that, in the cases where their spectra have sufficient $\mathrm{S} / \mathrm{N}$, the results fall within the same range of ages as their primary fits, suggesting that this is unlikely to be the main cause of the difference.

A second source of discrepancy may be that Canalizo \& Stockton deal with type I quasars, which requires that the apertures used in the modelling are offset from the nucleus by several kiloparsecs in order to avoid the overwhelming quasar nucleus. As simulations of galaxy mergers suggest that most of the merger-induced star formation will take place in the central regions of the galaxy on subkpc scales (e.g. di Matteo et al. 2005; Hopkins et al. 2013; Moreno et al. 2015), the inability to model the very central region may mean that the majority of this YSP is missed. However, in this context we note that some of the apertures that give young YSP ages in Mrk 231 (Canalizo \& Stockton 2001), J0025-10 (Bessiere et al. 2014) and J0218-00 (this work) are also significantly offset from the AGN nuclei.

A final basis for this discrepancy may be that type I and type II quasars are at different stages of their evolution. Once again, invoking the evolutionary sequence of Sanders et al. (1988), it is possible that the type II objects are being observed earlier in this sequence than the type I quasars, and would therefore have younger merger-induced YSPs. This scenario is supported by the findings of Kocevski et al. (2015) who have carried out a morphological study of AGN with a range of obscuration from Compton thick to unobscured. They find a significantly higher merger fraction in the obscured objects than the unobscured objects, which cannot be satisfactorily explained exclusively by the orientation-based unification scheme (Antonucci 1993). This might suggest that type I quasars are 'older' than type II quasars, and thus, the difference in the ages of the YSPs can be explained naturally.

Thanks to the quality and spectral range of these data, the type II quasars presented here are included in an expanding group of objects in which the starburst population can be reliably dated, with the clear detection of the strong Balmer absorption features in a large fraction of the sample ( $\sim 67$ per cent), and a significant contribution to the total flux by the YSP. These findings strongly emphasize that, in the cases in which the stellar populations can be unambiguously fitted, it is found that a significant proportion of the flux is attributable to a YSP $t_{\text {YSP }}<0.1 \mathrm{Gyr}$.

One of the goals of the stellar synthesis modelling presented in this work is to determine whether a delay between the peak of star formation and the triggering of AGN activity is observed in luminous AGN. Are the stellar populations of their host galaxies significantly older than the expected lifetime of the AGN? The answer to this question is largely dependent on the assumed lifetime of a typical quasar ( $\left.t_{\mathrm{QSO}}\right)$, which unfortunately is poorly constrained. Estimates, based on a variety of methods, are in the range $10^{6}<t_{\mathrm{QSO}}<10^{8} \mathrm{yr}$ (Martini 2004 and references therein). If the typical lifetime of a quasar is in fact closer to the lower limits (1 Myr), then the results presented here provide evidence for significant delay ( 50-100 Myr) between the peak of star formation and the emergence of the visible quasar activity in many of the sample objects. On the other hand, if the typical quasar lifetime is closer to the upper limit (50-100 Myr), as detailed simulations such as those of Hopkins et al. (2005) favour for the range for quasars of the luminosities considered here, then it can be concluded that the starburst and AGN activity are concurrent, with the possible exception of two objects $(\mathrm{J} 0848+01$ and J0948+00) that require YSP ages 200 Myr. Only when tighter constraints are placed on quasar lifetimes, will it be possible to answer this question with a degree of certainty.

\section{SUMMARY}

In this work, the stellar populations of a sample of 21 type II quasar host galaxies (including results presented by Bessiere et al. (2014) for J0025-10), selected from the SDSS (Zakamska et al. 2003) have been investigated. Our aim was to determine if YSP are present in these host galaxies, and if so, to establish their ages. In this way, we hope to gain valuable insights into the timing of AGN triggering and starbursts, and the sequence of events associated with galaxy mergers. We have done this using stellar population synthesis modelling, and also by the detection of Balmer absorption lines, which are an unambiguous marker of a YSP. Reddening of the YSP and AGN-related continuum components such as nebular continuum and scattered light have also been taken into account. It is important to consider these components, because failure to account for them may lead to an underestimation of the true ages of the YSPs. The results of the investigation can be summarized as follows.

(i) The Balmer absorption lines are clearly detected in $\sim 62$ per cent of the objects before the nebular continuum and higher order Balmer emission lines are subtracted, and in $\sim 67$ per cent of the objects after the subtraction.

(ii) $\sim 90$ per cent of the type II quasars cannot be adequately fitted using a combination of an OSP and power-law only. Therefore, they require a YSP component in order to produce an adequate fit.

(iii) In the majority of cases, a significant proportion of the flux in the normalizing bin is attributable to a YSP component. Values in the range 15-100 per cent have been found for the objects that can be successfully modelled using an $8 \mathrm{Gyr}+$ YSP combination of components.

(iv) Of the 24 apertures modelled, which can be fit using an 8 Gyr + YSP combination of components, 18 (75 per cent) have YSPs with a maximum acceptable age $t_{\max }=100 \mathrm{Myr}$, and 7 ( $\sim 30$ per cent) have a maximum acceptable age of $t_{\max }=50 \mathrm{Myr}$.

(v) Two objects (J0848+01 and J0948) require the inclusion of a YSP component with $t_{\mathrm{YSP}}>0.2 \mathrm{Gyr}$.

(vi) For an $8 \mathrm{Gyr}+$ YSP combination, 38 per cent of the apertures are strongly reddened $(E(B-V)>0.4)$, whilst 62 per cent of the apertures are not. There does not appear to be any correlation between YSP age and reddening.

We have also compared the stellar properties of type II quasar host galaxies to those of ULIRGs and PRGs, and find that the YSP ages of type II quasars are consistent with those found for both groups. However, the detection rate of YSPs in PRGs is only $\sim 23$ per cent (although this is a lower limit), whilst the detection rate for both the type II quasars and ULIRGs is $\sim 90$ per cent. This discrepancy in the detection rates of YSPs between PRGs and (in the vast majority of cases) radio-quiet type II quasars could suggest that the host galaxies of these two types of active systems are fundamentally different, whilst ULIRGs and type II quasars may form part of the same evolutionary sequence.

Although quasars selected for this study are type II, so that the direct quasar light is obscured from our direct view, scattered light from the quasar may nonetheless have an impact on the ages derived for the YSPs. Although the results summarized above assume that scattered light does not contribute significantly to the flux, we have explored the inclusion of a power-law component on the results 
of our modelling. We find that the inclusion of a power-law has no bearing on whether a YSP is detected; however, its major impact is to increase the range of ages and reddenings of the YSPs that produce acceptable fits, generally allowing for older ages. Clearly, future spectropolarimety observations - which explicitly quantify the level and spectrum of the scattered AGN component - are required to improve the accuracy of the determination of the YSP ages for the type II quasar host galaxies.

Our thorough investigation of the stellar properties of the host galaxies of type II quasars has unambiguously shown that YSPs are a fundamental constituent of a substantial proportion of objects. Moreover, because in most cases the maximum acceptable age of the YSP is comparable with the expected quasar lifetime, our study provides clear evidence that these type II quasars are being triggered quasi-simultaneously with the starburst activity, suggesting that both phenomena are being triggered at the peak of a galaxy merger.

\section{ACKNOWLEDGEMENTS}

First, we would like to acknowledge the valuable constructive feedback and suggestions of the referee. PSB acknowledges support from FONDECYT through grant 3160374. CRA acknowledges the Ramn y Cajal Programme of the Spanish Ministry of Economy and Competitiveness through project RYC-2014-15779. The main body of this work is based on observations obtained at the Gemini Observatory (acquired through the Gemini Observatory Archive and processed using the Gemini IRAF package), which is operated by the Association of Universities for Research in Astronomy, Inc., under a cooperative agreement with the NSF on behalf of the Gemini partnership: the National Science Foundation (United States), the National Research Council (Canada), CONICYT (Chile), Ministerio de Ciencia, Tecnología e Innovación Productiva (Argentina) and Ministério da Ciência, Tecnologia e Inovaçao (Brazil). The data were obtained under programmes GS-2010B-Q-83 and GS-2011BQ-42. We have also made use of observations made with the Gran Telescopio Canarias (GTC), installed in the Spanish Observatorio del Roque de los Muchachos of the Instituto de Astrofísica de Canarias, on the island of La Palma.

\section{REFERENCES}

Abazajian K. et al., 2004, AJ, 128, 502

Adelman-McCarthy J. K. et al., 2007, ApJS, 172, 634

Antonucci R., 1993, ARA\&A, 31, 473

Barth A. J., Voevodkin A., Carson D. J., Woźniak P., 2014, AJ, 147, 12

Bennert N., Canalizo G., Jungwiert B., Stockton A., Schweizer F., Peng C. Y., Lacy M., 2008, ApJ, 677, 846

Bessiere P., 2014, PhD thesis, Univ. Sheffield. Available at: http://etheses.whiterose.ac.uk/7696/

Bessiere P. S., Tadhunter C. N., Ramos Almeida C., Villar Martín M., 2012, MNRAS, 426, 276

Bessiere P. S., Tadhunter C. N., Ramos Almeida C., Villar Martín M., 2014, MNRAS, 438, 1839

Brotherton M. S., Grabelsky M., Canalizo G., van Breugel W., Filippenko A. V., Croom S., Boyle B., Shanks T., 2002, PASP, 114, 593

Bruzual G., Charlot S., 2003, MNRAS, 344, 1000

Calzetti D., Armus L., Bohlin R. C., Kinney A. L., Koornneef J., StorchiBergmann T., 2000, ApJ, 533, 682

Canalizo G., Stockton A., 2000, ApJ, 528, 201

Canalizo G., Stockton A., 2001, ApJ, 555, 719

Canalizo G., Stockton A., 2013, ApJ, 772, 132

Cardelli J. A., Clayton G. C., Mathis J. S., 1989, ApJ, 345, 245

Cid Fernandes R. et al., 2004, ApJ, 605, 105
Davies R. I., Müller Sánchez F., Genzel R., Tacconi L. J., Hicks E. K. S., Friedrich S., Sternberg A., 2007, ApJ, 671, 1388

di Matteo T., Springel V., Hernquist L., 2005, Nature, 433, 604

Dicken D. et al., 2014, ApJ, 788, 98

Dickson R., Tadhunter C., Shaw M., Clark N., Morganti R., 1995, MNRAS, 273, L29

Emonts B. H. C., Morganti R., Tadhunter C. N., Oosterloo T. A., Holt J., van der Hulst J. M., 2005, MNRAS, 362, 931

Emonts B. H. C., Morganti R., Tadhunter C. N., Holt J., Oosterloo T. A., van der Hulst J. M., Wills K. A., 2006, A\&A, 454, 125

Ferrarese L., Merritt D., 2000, ApJ, 539, L9

Floyd D. J. E., Kukula M. J., Dunlop J. S., McLure R. J., Miller L., Percival W. J., Baum S. A., O’Dea C. P., 2004, MNRAS, 355, 196

Gebhardt K. et al., 2000, ApJ, 539, L13

González Delgado R. M., Leitherer C., Heckman T. M., 1999, ApJS, 125, 489

Heckman T. M., Smith E. P., Baum S. A., van Breugel W. J. M., Miley G. K., Illingworth G. D., Bothun G. D., Balick B., 1986, ApJ, 311, 526

Heckman T. M., Gonzalez-Delgado R., Leitherer C., Meurer G. R., Krolik J., Wilson A. S., Koratkar A., Kinney A., 1997, ApJ, 482, 114

Heckman T. M., Ptak A., Hornschemeier A., Kauffmann G., 2005, ApJ, 634, 161

Holt J., Tadhunter C. N., Morganti R., 2003, MNRAS, 342, 227

Holt J., Tadhunter C., Morganti R., Bellamy M., González Delgado R. M., Tzioumis A., Inskip K. J., 2006, MNRAS, 370, 1633

Holt J., Tadhunter C. N., González Delgado R. M., Inskip K. J., Rodriguez Zaurin J., Emonts B. H. C., Morganti R., Wills K. A., 2007, MNRAS, 381,611

Hook I. M., Jørgensen I., Allington-Smith J. R., Davies R. L., Metcalfe N., Murowinski R. G., Crampton D., 2004, PASP, 116, 425

Hopkins P. F., Hernquist L., Cox T. J., Di Matteo T., Robertson B., Springel V., 2005, ApJ, 630, 716

Hopkins P. F., Hernquist L., Cox T. J., Di Matteo T., Robertson B., Springel V., 2006, ApJS, 163, 1

Hopkins P. F., Cox T. J., Hernquist L., Narayanan D., Hayward C. C., Murray N., 2013, MNRAS, 430, 1901

Kocevski D. D. et al., 2015, ApJ, 814, 104

Kroupa P., 2001, MNRAS, 322, 231

LaMassa S. M., Heckman T. M., Ptak A., Hornschemeier A., Martins L., Sonnentrucker P., Tremonti C., 2009, ApJ, 705, 568

Leitherer C. et al., 1999, ApJS, 123, 3

Liu X., Zakamska N. L., Greene J. E., Strauss M. A., Krolik J. H., Heckman T. M., 2009, ApJ, 702, 1098

Magorrian J. et al., 1998, AJ, 115, 2285

Martini P., 2004, Coevolution of Black Holes and Galaxies. Cambridge University Press, Cambridge

Martini P., Weinberg D. H., 2001, ApJ, 547, 12

Moreno J., Torrey P., Ellison S. L., Patton D. R., Bluck A. F. L., Bansal G., Hernquist L., 2015, MNRAS, 448, 1107

Obied G., Zakamska N. L., Wylezalek D., Liu G., 2016, MNRAS, 456, 2861

Ramos Almeida C., Tadhunter C. N., Inskip K. J., Morganti R., Holt J., Dicken D., 2011, MNRAS, 410, 1550

Ramos Almeida C. et al., 2012, MNRAS, 419, 687

Ramos Almeida C., Rodríguez Espinosa J. M., Acosta-Pulido J. A., AlonsoHerrero A., Pérez García A. M., Rodríguez-Eugenio N., 2013, MNRAS, 429, 3449

Reyes R. et al., 2008, AJ, 136, 2373

Robinson T., 2001, PhD thesis, Univ. Sheffield

Robinson T. G., Tadhunter C. N., Axon D. J., Robinson A., 2000, MNRAS, 317,922

Rodríguez Zaurín J., Tadhunter C. N., González Delgado R. M., 2009, MNRAS, 400, 1139

Rodríguez Zaurín J., Tadhunter C. N., González Delgado R. M., 2010, MNRAS, 403, 1317

Sanders D. B., Soifer B. T., Elias J. H., Madore B. F., Matthews K., Neugebauer G., Scoville N. Z., 1988, ApJ, 325, 74

Schlegel D. J., Finkbeiner D. P., Davis M., 1998, ApJ, 500, 525

Schneider D. P. et al., 2010, AJ, 139, 2360 
Seaton M. J., 1979, MNRAS, 187, 73P

Shen Y. et al., 2011, ApJS, 194, 45

Springel V., Di Matteo T., Hernquist L., 2005, MNRAS, 361, 776

Swinbank A. M., Balogh M. L., Bower R. G., Zabludoff A. I., Lucey J. R., McGee S. L., Miller C. J., Nichol R. C., 2012, MNRAS, 420, 672

Tadhunter C. N., Morganti R., di Serego-Alighieri S., Fosbury R. A. E., Danziger I. J., 1993, MNRAS, 263, 999

Tadhunter C. N., Morganti R., Robinson A., Dickson R., Villar-Martin M., Fosbury R. A. E., 1998, MNRAS, 298, 1035

Tadhunter C., Dickson R., Morganti R., Robinson T. G., Wills K., VillarMartin M., Hughes M., 2002, MNRAS, 330, 977

Tadhunter C., Robinson T. G., González Delgado R. M., Wills K., Morganti R., 2005, MNRAS, 356, 480

Tadhunter C. et al., 2011, MNRAS, 412, 960

Tran K.-V. H., Franx M., Illingworth G., Kelson D. D., van Dokkum P., 2003, ApJ, 599, 865

Tran K.-V. H., Franx M., Illingworth G. D., van Dokkum P., Kelson D. D., Magee D., 2004, ApJ, 609, 683

Treister E., Schawinski K., Urry C. M., Simmons B. D., 2012, ApJ, 758, L39

Villar-Martín M., Cabrera Lavers A., Bessiere P., Tadhunter C., Rose M., de Breuck C., 2012, MNRAS, 423, 80

Wild V., Heckman T., Charlot S., 2010, MNRAS, 405, 933

Wills K. A., Tadhunter C., Holt J., González Delgado R., Inskip K. J., Rodríguez Zaurín J., Morganti R., 2008, MNRAS, 385, 136

Wylezalek D., Zakamska N. L., Liu G., Obied G., 2016, MNRAS, 457, 745

York D. G. et al., 2000, AJ, 120, 1579

Zakamska N. L. et al., 2003, AJ, 126, 2125

\section{APPENDIX A: RESULTS FOR INDIVIDUAL OBJECTS}

In this appendix, a brief description of the stellar synthesis modelling for each of the objects is outlined. We also present examples of acceptable fits for each of the objects, showing the fit to both the overall spectrum, and also in the key Balmer absorption region. J0025-10 is discussed in detail in Bessiere et al. (2014).

\section{A1 J2358-00}

The initial modelling for J2358-00 was performed using combination 2 , and resulted in a best fitting model with $t_{\mathrm{YSP}}=0.02 \mathrm{Gyr}$ and $E(B-V)=0.5$. The model nebular continuum contributes $\sim 28$ per cent of the total flux below the Balmer edge. The reddening derived from the $\mathrm{H} \gamma / \mathrm{H} \beta$ and $\mathrm{H} \delta / \mathrm{H} \beta$ ratio is consistent with Case B (Table 4), and therefore, no reddening was applied before subtraction. The resulting spectrum shows no evidence of an unphysical step at the Balmer edge, thus was deemed acceptable.

All five modelling combinations were utilized in order to perform the stellar synthesis modelling, and the results presented in Tables 5 and 6 show that all models that include a YSP component produce acceptable solutions.

It was possible to reject a significant number of potential solutions based on the strong overprediction of $\mathrm{Ca}$ II $\mathrm{K}$ absorption. In fact, the bottom panel of Fig. A1 shows that this feature is unusually weak in the spectrum of $\mathbf{J} 2358-00$, although the Balmer lines are clearly detectable. The reason for this is not immediately apparent but is not due to any cosmetic defects in the spectrum at this wavelength. This feature provides a strong constraint on the acceptable ages and reddenings of the YSP when no power-law component is included $\left(0.04 \leq t_{\mathrm{YSP}}(\mathrm{Gyr}) \leq 0.05\right)$, with reddenings in the range $0.5 \leq$ $E(B-V) \leq 0.6$. However, when a power-law component is included, a much wider range of ages and reddenings becomes permissible, although in a majority of cases, the YSP flux is dominant over the
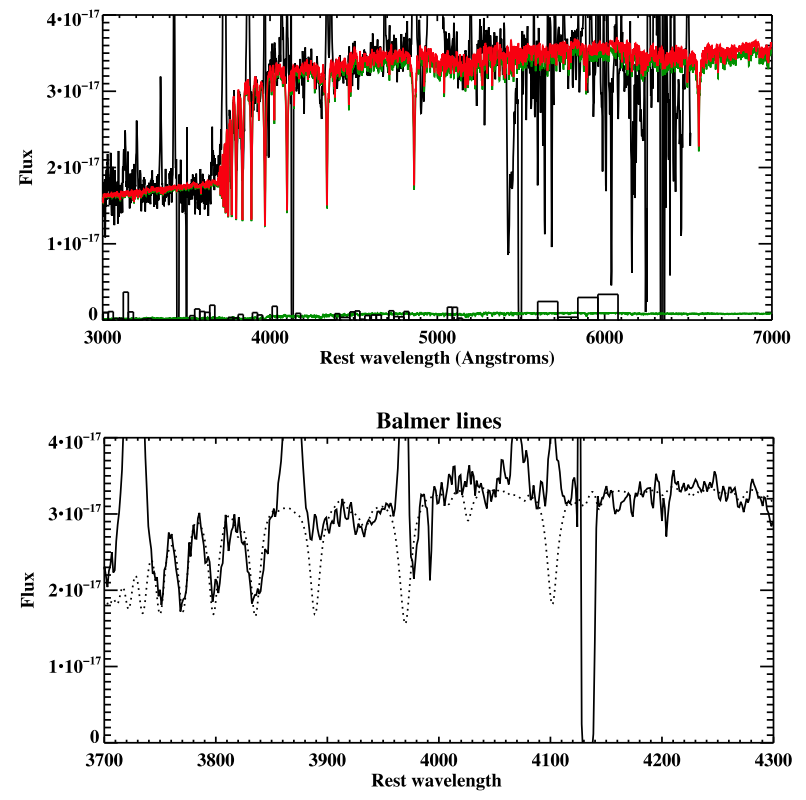

Figure A1. An example of an acceptable fit produced by CONFIT for J2358-00. The fit shown here was produced using combination 2 and includes a YSP with $t_{\mathrm{YSP}}=0.04 \mathrm{Gyr}$ and $E(B-V)=0.6$.

OSP. Fig. A1 shows an example of an acceptable fit generated using combination 2.

\section{A2 J0114+00}

Unfortunately, no Gemini GMOS-S spectrum is available for J0114+00, and therefore, we model the SDSS spectrum instead. Although this spectrum has a lower S/N than the Gemini GMOS-S data, it is still of adequate quality and covers a sufficient wavelength range to perform spectral synthesis modelling.

After performing the initial run (combination 2), the stellar model, which included a YSP with $t_{\mathrm{YSP}}=0.04 \mathrm{Gyr}$ and $E(B-V)$ $=0.8$ was subtracted. The model nebular continuum then contributes $\sim 15$ per cent of the total flux bluewards of the Balmer edge (Table 4). In this case, it was not possible to make a reliable estimate of the Balmer decrements, because of the intrinsically low equivalent widths of the $\mathrm{H} \gamma$ and $\mathrm{H} \delta$ emission lines, and the low $\mathrm{S} / \mathrm{N}$ of these data. Therefore, no reddening correction was applied to the model nebular continuum before subtraction.

Tables 5 and 6 demonstrate that for this particular object, the OSP dominates the flux in the normalizing bin, which leads to the models being relatively unconstrained. Therefore, the fits produce a wide range of ages and reddenings $\left(0.004 \leq t_{\mathrm{YSP}}(\mathrm{Gyr}) \leq 0.4\right.$ and $0.6 \leq$ $E(B-V) \leq 1.3)$ for combination 2. Fig. A2 shows an example of an acceptable fit generated by combination 2 with $t_{\mathrm{YSP}}=0.06 \mathrm{Gyr}$ and $E(B-V)=0.9$.

\section{A3 J0123+00}

The initial modelling for $\mathrm{J} 0123+00$ was performed using combination 4 , because it was not possible to obtain acceptable fits without the inclusion of a power-law component. However, this resulted in a wide range of ages and reddenings producing acceptable fits $\left(2 \leq t_{\mathrm{ISP}}(\mathrm{Gyr}) \leq 5\right.$ and $\left.0.4 \leq E(B-V) \leq 1.2\right)$, which, based on agesensitive stellar features, were impossible to differentiate between. Therefore, the stellar model that produced the lowest $\chi_{\text {red }}^{2}$ value was 

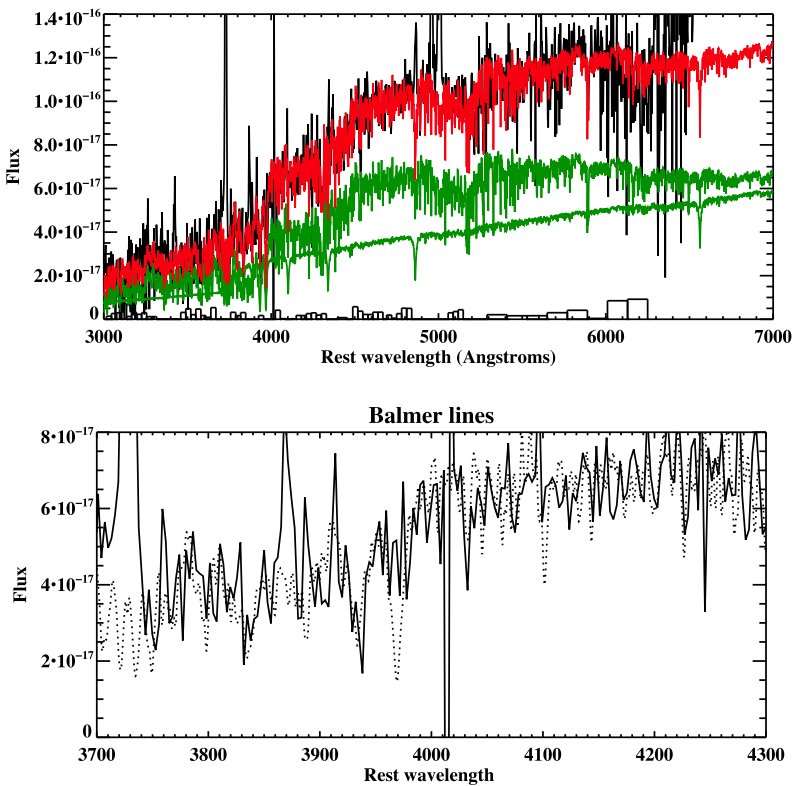

Figure A2. An example of an acceptable fit produced by CONFIT for J00114+00. This fit is produced by combination 2 and includes a YSP with $t_{\mathrm{YSP}}=0.06 \mathrm{Gyr}$ and $E(B-V)=0.9$.

subtracted, which included an ISP with $t_{\mathrm{ISP}}=5.0 \mathrm{Gyr}, E(B-V)=$ 0.6 and a power-law component.

The nebular continuum model produces a large contribution to the total flux bluewards of the Balmer edge ( $\sim 48$ percent), and subtraction of this model without first applying reddening of the value derived from the Balmer line ratios (Table 4), results in an unphysical step in the spectrum. Therefore, the model continuum was reddened using a value of $E(B-V)=0.2$, which is the mean value of reddening derived from the $\mathrm{H} \gamma / \mathrm{H} \beta$ and $\mathrm{H} \delta / \mathrm{H} \beta$ ratios. The reddened nebular continuum contributes $\sim 20$ percent of the flux just below the Balmer edge, and does not result in an over subtraction of the nebular continuum component.

Tables 5 and 6 show that, when an 8 Gyr population is combined with a YSP model and a power-law component, it is possible to achieve an acceptable fit to the spectra, but not with the inclusion of an $2 \mathrm{Gyr}$ population. When attempting to perform the stellar population modelling for $\mathrm{J} 0123+00$, it has proven difficult to constrain the ages of the YSP/ISP component. Once again (as for J0114+00), this may be due to the fact that the flux is dominated by the OSP for nearly all possible acceptable solutions, making it difficult to accurately model the lower level YSP contribution.

Fig. A3 shows an example of an acceptable fit using combination 4 , which in this case include a young component of $t_{\mathrm{ISP}}=4 \mathrm{Gyr}$ and $E(B-V)=0.3$.

\section{A4 J0142+14}

Unlike the other objects for which GMOS-S spectroscopic data are available, for J0142+14 only the blue part of the spectrum was observed (Table 2). However, modelling of this object is still well constrained because the spectrum has a high $\mathrm{S} / \mathrm{N}$ as well as strong Balmer absorption lines. The rest-frame spectral range covered by this spectrum is $2813-4863 \AA$, meaning we are unable to recover the $\mathrm{H} \beta$ flux and generate a nebular continuum model.

Tables 5 and 6 show that all the modelling combinations including a YSP component were successful. In all cases, where an adequate fit was achieved, the allowable ages are very similar $\left(0.04 \leq t_{\mathrm{YSP}}(\mathrm{Gyr})\right.$
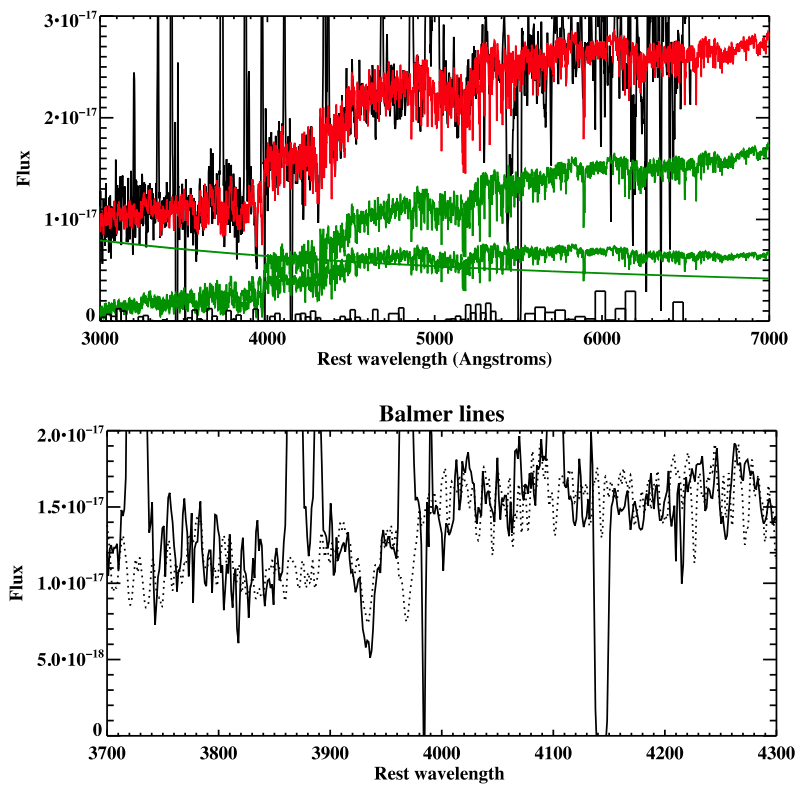

Figure A3. An example of an acceptable fit produced by confiT for $\mathrm{J} 0123+00$. This fit is generated by combination 6 and includes an ISP with $t_{\mathrm{ISP}}=4$ and $E(B-V)=0.3$.
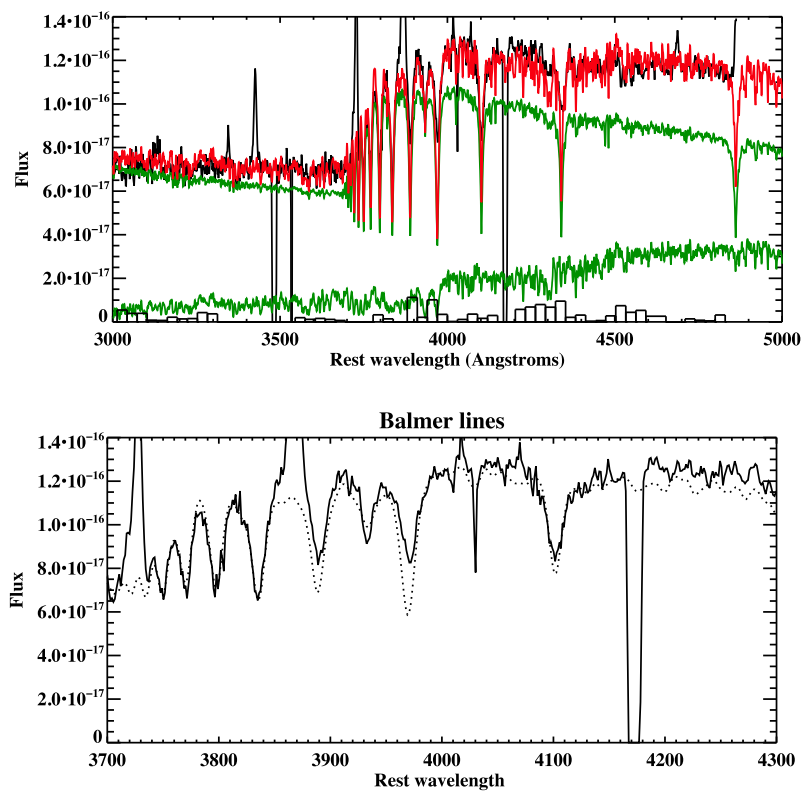

Figure A4. An example of an acceptable fit produced by CONFIT for $\mathrm{J} 0142+14$. This fit is produced by combination 2 and includes a YSP with $t_{\mathrm{YSP}}=0.08$ Gyr with $E(B-V)=0.2$.

$\leq 0.1$, except in the case of combination 4 , where the maximum allowable age is $0.4 \mathrm{Gyr}$. In all cases, the reddening of the YSP is $0.1 \leq E(B-V) \leq 0.5$, and the flux of the YSP dominates the total flux of the system, in some cases accounting for 100 per cent of the flux.

It is interesting to note that $\mathrm{J} 0142+14$ is classified as undisturbed in terms of its morphology, and appears to be relatively compact in the deep $r^{\prime}$-band image presented in Bessiere et al. (2012). However, the modelling results presented here combined with the clearly detectable Balmer absorption lines in the spectrum (illustrated in Fig. A4) make it clear that there has been a powerful episode of 
star formation within the last $0.4 \mathrm{Gyr}$. This suggests that $\mathrm{J} 0142+14$ may be an 'E+A' galaxy, which is thought to be a short-lived phase in which a galaxy transforms from star-forming to passively evolving (Tran et al. 2003, 2004). These galaxies are characterized by weak nebular lines (associated with star formation) such as [O II] $\lambda 3727$ and strong absorption lines, such a $\mathrm{H} \delta$, characteristics that are clearly visible in the spectrum of J0142+14 (Fig. 1).

Swinbank et al. (2012) present integral field unit observations for $11 \mathrm{E}+\mathrm{A}$ galaxies $(0.07<z<0.12)$ and find that 90 per cent of their sample have residual rotational motion, with the A-type stars spread throughout the galaxy, a finding that they attribute to previous minor/major merger activity. It is plausible that this is also the case for J0142+14 implying that, even though no evidence of a recent merger is detected, it is likely that one has occurred fairly recently. Fig. A4 shows an example of a fit produced using combination 2, which includes a YSP of $t_{\mathrm{YSP}}=0.08 \mathrm{Gyr}$ with $E(B-V)=0.2$.

\section{A5 J0217-00}

After the initial modelling of J0217-00 (using combination 2), a stellar model with a YSP $t_{\mathrm{YSP}}=0.05 \mathrm{Gyr}$ and $E(B-V)=0.5$ was selected for subtraction, resulting in a nebular continuum that contributes $\sim 14$ percent of the total flux just below the Balmer edge (3540-3640 $)$ ). In this case, it was not possible to determine any values for the Balmer decrements because $\mathrm{H} \gamma$ falls directly adjacent to a chip gap, and the low equivalent width of $\mathrm{H} \delta$ results in uncertainties that are larger than the measured values. Therefore, no reddening correction was applied to the nebular continuum before subtraction; however, this did not result in an unphysical step at the Balmer edge.

Tables 5 and 6 show that all combinations that include a YSP are successful, and that for both cases in which the spectrum was modelled with no power-law component (combinations 2 and 3), the acceptable ages are in the range $0.06 \leq t_{\mathrm{YSP}}(\mathrm{Gyr}) \leq 0.1$ and the associated values of reddening are in the range $0.5 \leq E(B-V) \leq 0.6$. When a power-law component was also included, the range of acceptable ages in both cases is extended to $0.4 \mathrm{Gyr}$. Fig. A5 shows an example of an acceptable fit generated by combination 2 .

\section{A6 J0217-01}

The initial modelling of J0217-01 was performed using combination 2, and produced a best-fitting model that included a YSP of age $t_{\mathrm{YSP}}=0.2$ and $E(B-V)=0.9$, which resulted in a nebular continuum model that contributes $\sim 5$ per cent of the total flux just below the Balmer edge. Attempting to derive the Balmer decrements proved unsuccessful because of the large errors associated with the $\mathrm{H} \gamma$ and $\mathrm{H} \delta$ measurements, which are ultimately due to this weak nebular continuum.

All five combinations of components were applied, and of these, only combinations that include an $8 \mathrm{Gyr}$ unreddened component and a YSP (combinations 2 and 4) produced acceptable fits (Table 5). In the majority of cases where acceptable solutions are found, the flux is dominated by the OSP component. Fig. A6 shows an example of an acceptable fit to J0217-01, produced by combination 2, which includes a YSP of $t_{\mathrm{YSP}}=0.007 \mathrm{Gyr}$ and $E(B-V)=0.9$.

\section{A7 J0218-00}

J0218-00 is a complex system, with two distinct nuclei that have a projected separation of $\sim 12 \mathrm{kpc}$ (Bessiere et al. 2012). This interaction has left the quasar host galaxy in what appears to be a ring-like
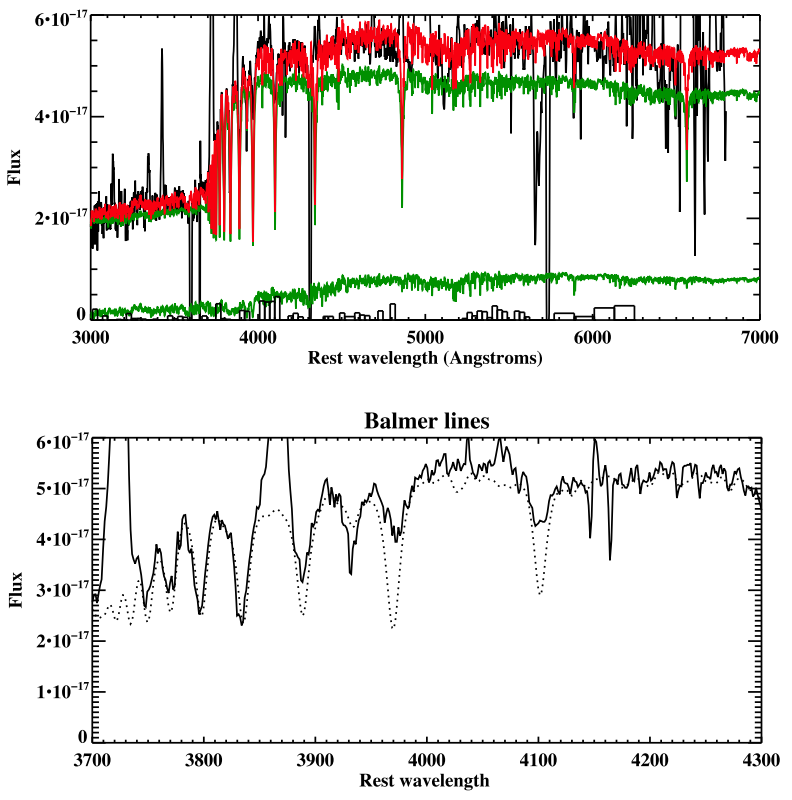

Figure A5. An example of an acceptable fit produced by CONFIT for J0217-00. This fit was produced by using combination 2 and includes a YSP with $t_{Y S P}=0.1$ and $E(B-V)=0.5$.
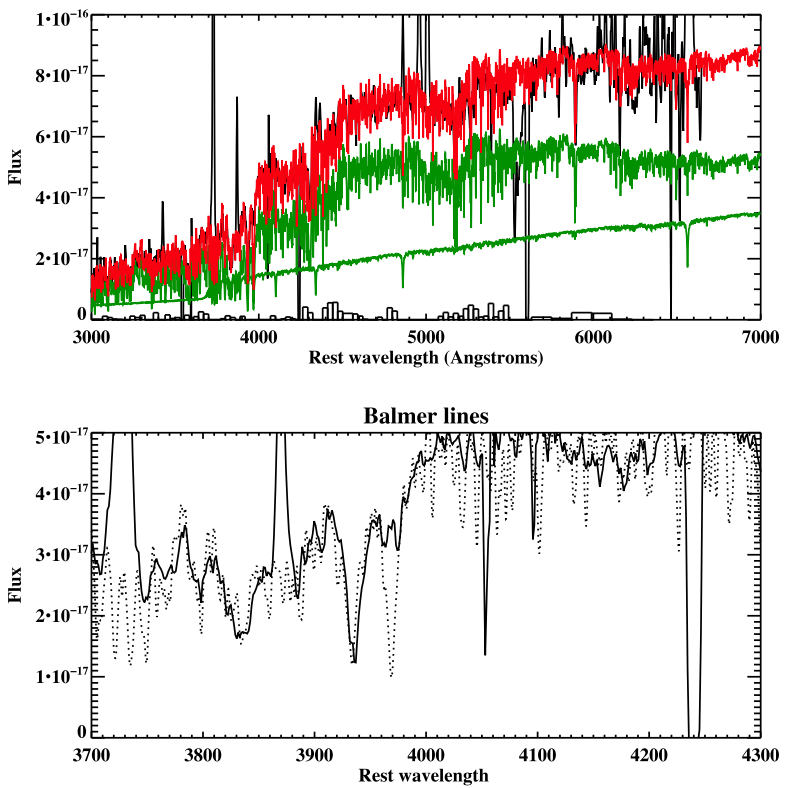

Figure A6. An example of an acceptable fit produced by CONFIT for J0217-01. This fit was produced by combination 2 and incorporates a YSP with $t_{\mathrm{YSP}}=0.007 \mathrm{Gyr}$ and $E(B-V)=0.9$.

configuration. The spectroscopic slit does not pass through both nuclei, yet it was still possible to extract two distinct apertures from the quasar host galaxy. The main aperture is centred on the region containing the active nucleus (with a spatial scale of $8 \mathrm{kpc}$ ) and a second, narrower aperture is centred on an extended region of the ring-like structure (with a spatial scale of $5 \mathrm{kpc}$ ). The approximate positions of the apertures is shown in Fig. A7 where they have been overlaid on the image. A cross-section of the $2 \mathrm{D}$ spectrum is also shown, showing the exact position of each aperture. The centres of the two apertures are separated by $\sim 8.2 \mathrm{kpc}$. Each aperture was 

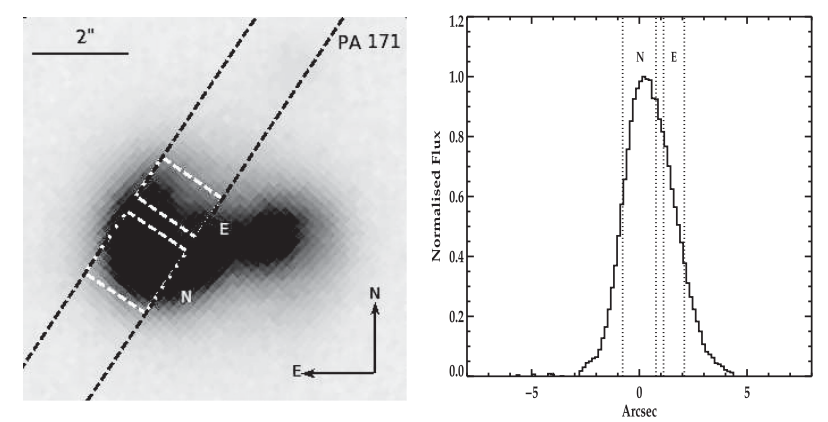

Figure A7. The left-hand panel shows our Gemini GMOS-S image of J0218-00 with the spectroscopic slit overlaid. The positions of the two extracted apertures are also shown. The right-hand panel shows a crosssection of the 2D spectrum of J0218-00 showing the position of each extracted aperture. The cross-section was extracted at an observed wavelength of 6450-6600 A (4700-4810 ̊ rest-frame.)

modelled independently, and the results are presented separately below.

\section{A7.1 Nuclear aperture}

The initial fit for the nuclear aperture was performed using combination 2, and a best fitting model, which included a YSP $t_{\text {YSP }}=0.03 \mathrm{Gyr}$ and $E(B-V)=0.1$, was selected. This was used to generate a nebular continuum that contributes $\sim 16$ per cent of the total flux in the $3540-3640 \AA$ range. The $\mathrm{H} \gamma / \mathrm{H} \beta$ ratio was measured to be $0.37 \pm 0.02$ (Table 4 ), and the reddening derived from this ratio was initially applied to the nebular continuum before subtraction. However, this resulted in an undersubtraction in the region of the Balmer edge, and therefore, an unreddened nebular continuum was subtracted instead, which produced a reasonable result. The failure of the technique in this case is most likely due to the relatively low equivalent width of the Balmer emission lines and strong underlying Balmer absorption lines. It is probably the case that the true value of reddening is somewhere between these extreme cases. However, it is difficult to judge what the most appropriate value is by visual inspection alone.

All five combinations of components were run for the nuclear aperture, the results of which are given in Tables 5 and 6 . These result show that, when no power-law component is included, it was only possible to achieve acceptable fits for combination 2 . Combinations that included a power-law component and a YSP all produced acceptable fits (combinations 4 and 5). Fig. A8 shows an example of an acceptable fit produced by combination 2 , which has a YSP of $t_{\mathrm{YSP}}=0.04 \mathrm{Gyr}$ and $E(B-V)=0.2$.

\section{A7.2 Extended aperture}

The initial modelling for the extended aperture extracted from J0218-00 was performed using combination 2, which resulted in a best-fitting model with $t_{\mathrm{YSP}}=0.03$ and $E(B-V)=0.2$. This is very similar in terms of the components used, and their contribution to the total flux as for the nuclear aperture. This resulted in a model nebular continuum that contributes $\sim 16$ per cent of the total flux in the 3540-3640 A range. The measured Balmer decrement produced a value of $\mathrm{H} \gamma / \mathrm{H} \beta=0.32 \pm 0.02$, suggesting that the nebular continuum is slightly more reddened in the extended region than in the nuclear region (see Table 4). However, when the continuum was reddened before subtraction, the same issues was encountered as
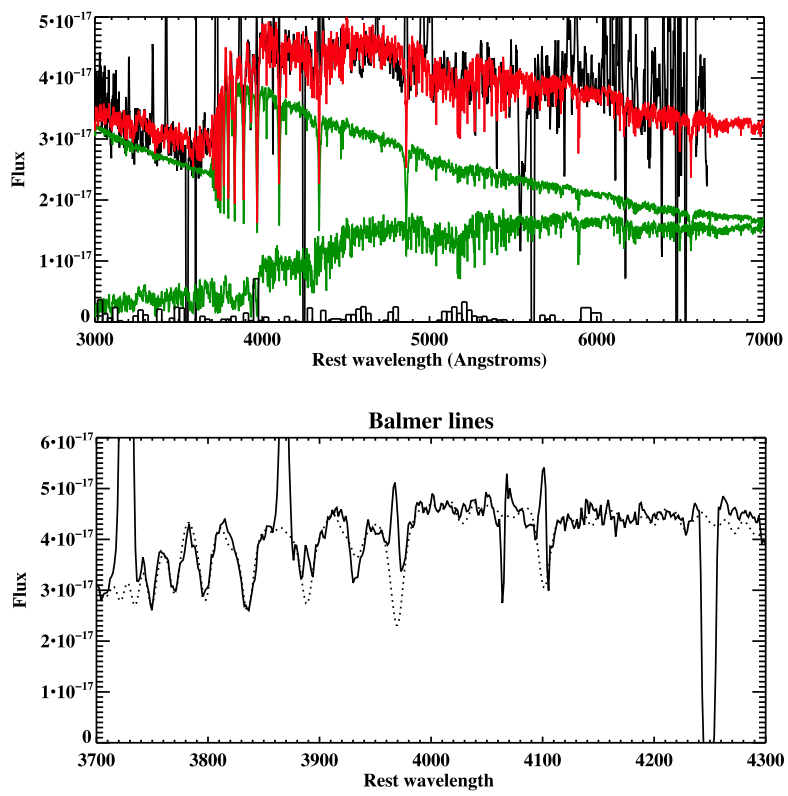

Figure A8. An example of an acceptable fit produced by CONFIT for J0218-00 - nuclear aperture. This fit was generated using combination 2 and includes a YSP with $t_{\mathrm{YSP}}=0.04 \mathrm{Gyr}$ and $E(B-V)=0.2$.
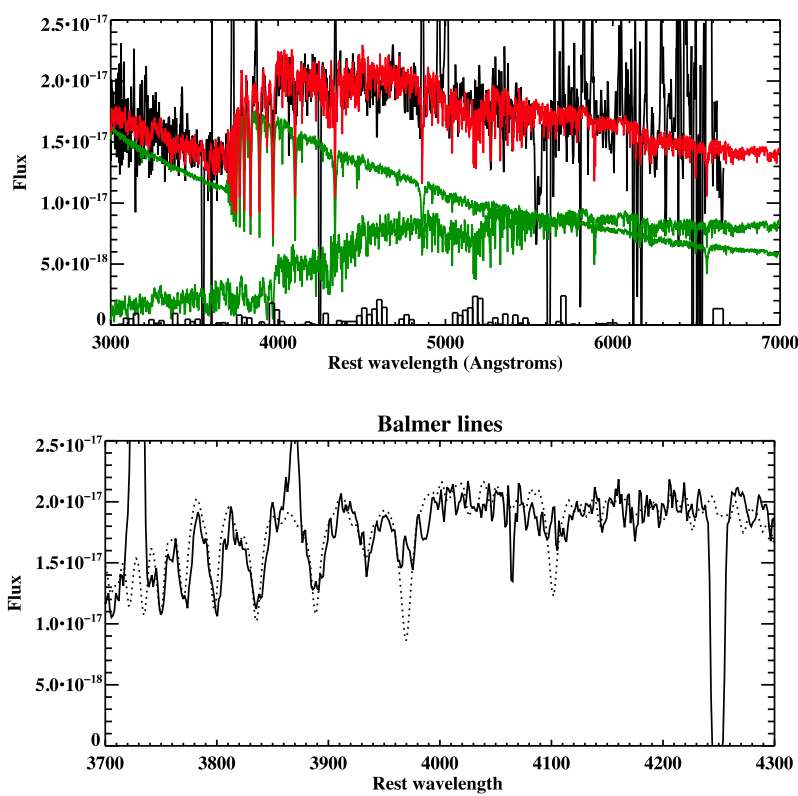

Figure A9. An example of an acceptable fit produced by CONFIT for J0218-00 - extended aperture. This model was generated using combination 2 and includes a YSP with $t_{\mathrm{YSP}}=0.04$ and $E(B-V)=0.1$.

described for the nuclear aperture. Therefore, an unreddened continuum was subtracted before continuing with the full modelling process.

The extended aperture is centred on a non-active, extended region of the galaxy and therefore, only combinations 2 and 3 (no powerlaw component) were used for the modelling process. Fig. A9 shows an example of an acceptable fit for combination 2, which includes a YSP $t_{\mathrm{YSP}}=0.04 \mathrm{Gyr}$ and $E(B-V)=0.1$. It can be seen by comparison with Fig. A8 that the overall shape and the age-sensitive absorption lines in the region of the higher order Balmer lines are 

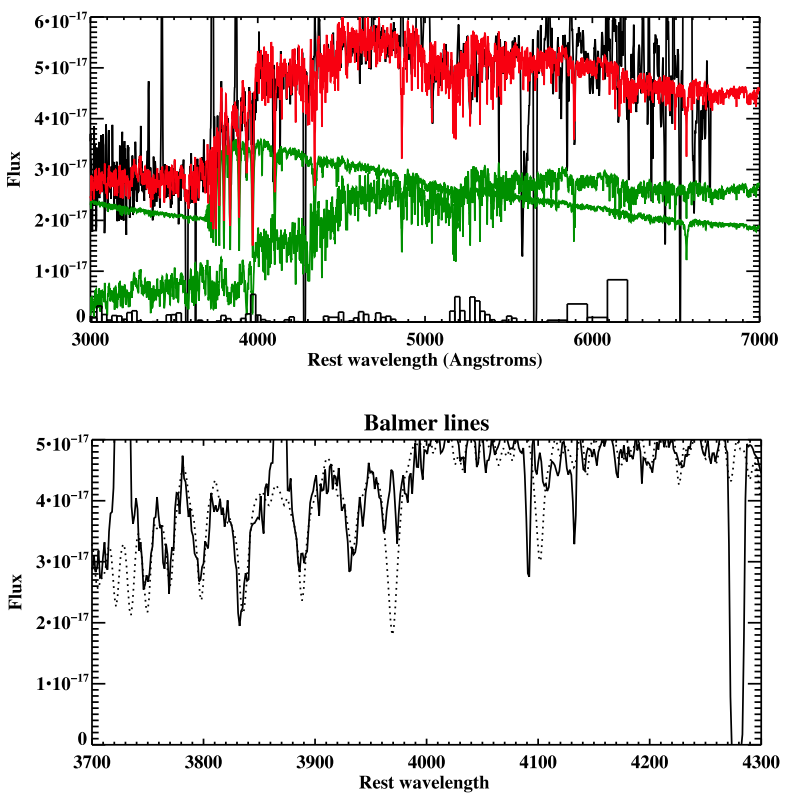

Figure A10. An example of an acceptable fit produced by CONFIT for J0227+01. This fit was generated using combination 2 and consists of an $8 \mathrm{Gyr}$ OSP combined with a YSP of $t_{\mathrm{YSP}}=0.05 \mathrm{Gyr}$ and $E(B-V)=$ 0.3 .

very similar, which may suggest that the burst of star formation induced by this obvious merger was initiated across the entire galaxy.

\section{A8 J0227+01}

The initial modelling for $\mathrm{J} 0227+01$ was performed using combination 2 , which resulted in a best fitting model with $t_{\mathrm{YSP}}=0.04 \mathrm{Gyr}$ and $E(B-V)=0.6$. This resulted in a nebular continuum model that contributes $\sim 11$ per cent of the total flux just below the Balmer edge (Table 4). Unsurprisingly, with such a comparatively weak nebular continuum, both the $\mathrm{H} \gamma$ and $\mathrm{H} \delta$ emission lines are weak, preventing a reliable measurement of the nebular reddening, via the Balmer decrements, being made. In light of this, it was not possible to apply a reddening correction to the nebular component before subtraction. However, the resulting nebular subtracted spectrum produced an acceptable result.

All five combination of components were then run for $\mathrm{J} 0227+01$, and the results, presented in Tables 5 and 6 , show that the full range of combinations that include a YSP produce an acceptable fit. When combinations that do not include a power-law component are used, the range of ages that produce acceptable fits is $0.03 \leq t_{\mathrm{YSP}} \leq$ 0.1 , with reddenings in the range $0.1 \leq E(B-V) \leq 0.5$ and the YSP component contributing up to 85 per cent of the flux in the normalizing bin. When a power-law component is also included, the ages move to older values $\left(0.09 \leq t_{\mathrm{YSP}} \leq 0.2\right)$ and a larger range of reddenings is allowed $(0 \leq E(B-V) \leq 0.6)$. Fig. A10 shows an example of an acceptable fit, using combination 2, which includes a YSP with $t_{\mathrm{YSP}}=0.05 \mathrm{Gyr}$ and $E(B-V)=0.3$.

\section{A9 $\mathbf{J 0 2 3 4 - 0 7}$}

The initial modelling for J0234-07 was performed using combination 2 and resulted in a wide range of possible solutions that have $\chi_{\text {red }}^{2}<1$. An inspection of the overall shape of the continuum and regions around age-sensitive stellar absorption lines showed the best fit to have a YSP $t_{\mathrm{YSP}}=0.001$ and $E(B-V)=0.1$. How-
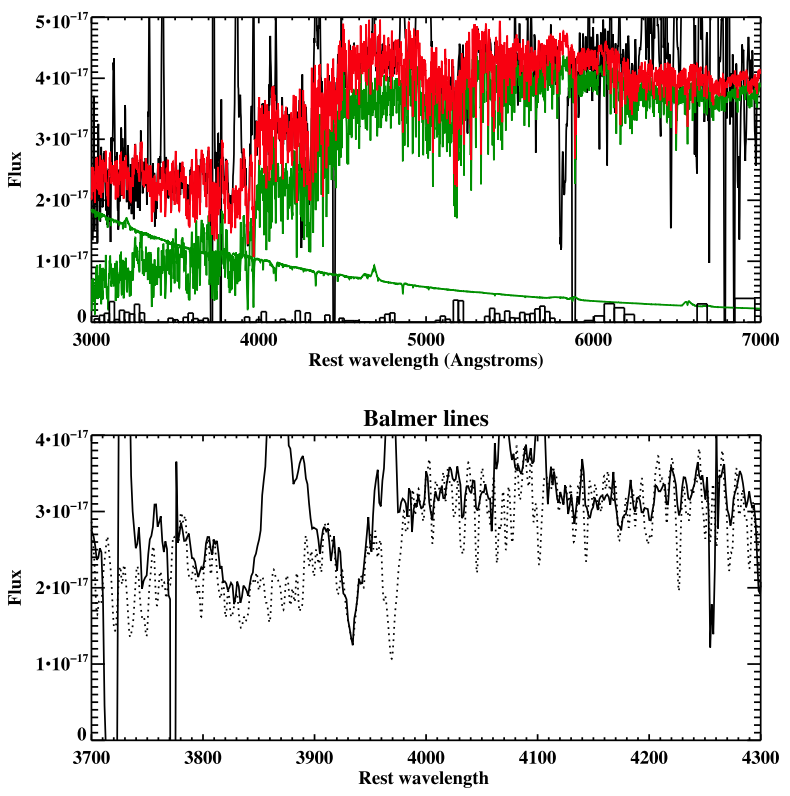

Figure A11. An example of an acceptable fit produced by CONFIT for J0234-07. The fit shown here was generated using combination 2 and includes a YSP with $t_{\mathrm{YSP}}=0.003 \mathrm{Gyr}$ and $E(B-V)=0.2$.

ever, Table 5 shows that the OSP dominates the flux for all other possible solutions for combination 2, and therefore, the resulting stellar model is not strongly dependent on the choice of YSP component. A nebular continuum model that contributes $\sim 24$ per cent of the flux in the 3540-3640 $\AA$ range was constructed. Initially, to remain consistent with the technique of maximum nebular subtraction applied in the majority of cases, no reddening was applied to the nebular continuum before subtraction. However, this resulted in a step in the resulting stellar continuum at $3646 \AA$. To resolve this issue, reddening of $E(B-V)=0.25$ was applied to the nebular continuum before subtraction (Table 4 ), which produced a much improved result.

All five combinations were run, and all but combination 3 produce acceptable results. It should be noted however that all possible YSP and reddening values for combination 4 produce acceptable results, and therefore, do not allow us to discriminate between solutions. In addition to combinations that include a YSP, J0234-07 can also be fitted using only an $8 \mathrm{Gyr}$, unreddened population combined with a power-law component, making it unclear whether a YSP component is at all necessary. Tables 5 and 6 show that the combinations that do not include a power-law component tend to include very young or young components at a low level of contribution to the total flux in the normalizing bin. This means that in these cases, the VYSP/YSP could, in fact, be taking the form of a power-law, as illustrated in Fig. A11, where the VYSP $\left(t_{\mathrm{YSP}}=0.003, E(B-V)=0.2\right)$ has an approximately power-law form.

With such a wide range of possible solutions and such young ages producing acceptable fits to these data, as well as an 8 Gyr plus power-law combination, it is not possible to discriminate between the various possibilities. However, there is little evidence for the Balmer absorption lines, associated with young stars (Fig. A11b), although these are also weak at the youngest model ages.

\section{A10 J0249-00}

In order to produce a stellar model, the initial run was performed using combination 2 . This resulted in a best-fitting model with 

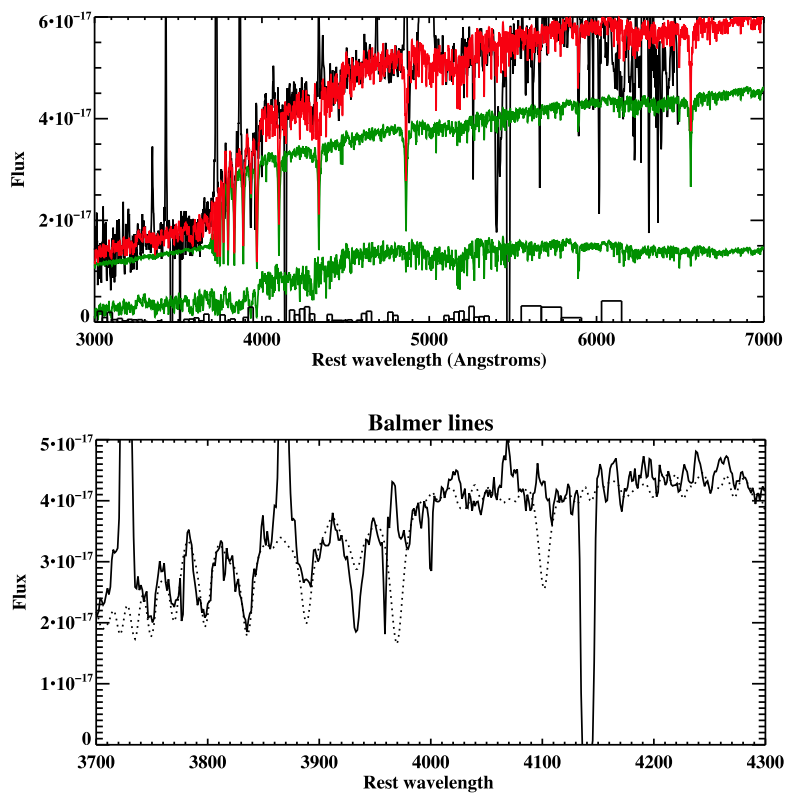

Figure A12. An example of an acceptable fit produced by CONFIT for J0249-00. The fit shown here was generated by combination 2 and shows a YSP with $t_{\mathrm{YSP}}=0.07 \mathrm{Gyr}$ and $E(B-V)=0.7$.

$t_{\mathrm{YSP}}=0.03$ and $E(B-V)=0.7$ and produced a model nebular continuum, which contributes $\sim 25$ per cent of the flux in the 3540 $3640 \AA$ range. No reddening was applied to this continuum because, as is shown in Table 4, the uncertainty associated with measuring the $\mathrm{H} \gamma / \mathrm{H} \beta$ ratio is large in comparison to the measured value, and thus is consistent with reddenings of $0 \leq E(B-V) \leq 1.7$. Therefore, because the value of reddening is poorly constrained, and also because subtracting an unreddened nebular continuum provides an acceptable result, a maximum nebular subtraction was performed before proceeding with the fitting.

Of the five combinations of components, all those which include a YSP produce acceptable results. Tables 5 and 6 show that, in the cases of combinations 2 and 3 , the ages and reddenings allowed are broadly similar, with combination 3 allowing a slightly older age and larger range of reddenings. As is typical, when a power-law component is included, significantly older ages provide acceptable fits at a wider range of reddenings. Fig. A12 shows an example of an acceptable fit using combination 2 , and includes a YSP of $t_{\mathrm{YSP}}=$ 0.07 Gyr and $E(B-V)=0.7$.

\section{A11 J0320+00}

The initial modelling for $\mathrm{J} 0320+00$ was performed using combination 2, and the resulting best-fitting model includes a YSP of $t_{\mathrm{YSP}}=$ 0.02 Gyr and $E(B-V)=0.1$ and a model nebular continuum that contributes $\sim 14$ per cent of the flux in the 3540-3640 $\AA$ range was generated. The Balmer decrements were measured using $\mathrm{H} \beta$ and $\mathrm{H} \gamma$ (Table 4) but because of the large uncertainty associated with the measured value, which allows for reddenings in the range $0 \leq$ $E(B-V) \leq 1.3$, and the fact that the maximum subtraction produces a good result around the Balmer edge, no reddening correction has been applied to the nebular continuum model before subtraction.

The results of running combinations 2-5 are presented in Tables 5 and 6 , which show that only combination 3 , which includes an underlying 2 Gyr ISP, does not provide acceptable solutions. Columns 5 and 6 of Tables 5 and 6 demonstrate that, when no power law
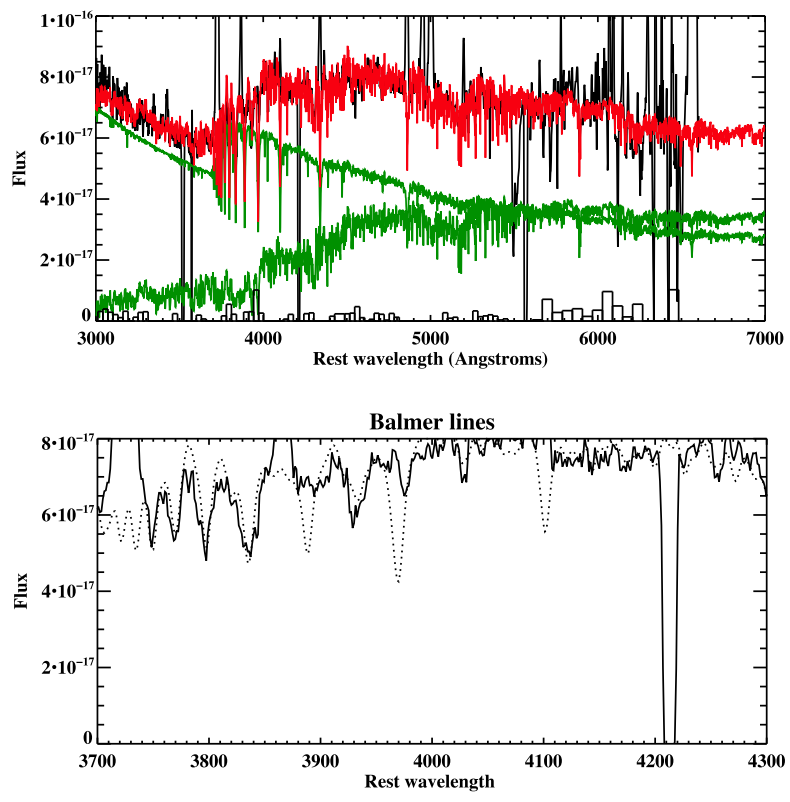

Figure A13. An example of an acceptable fit produced by CONFIT for J0320-00. The fit shown here was generated using combination 2, and includes a YSP with $t_{\mathrm{YSP}}=0.02$ and $E(B-V)=0.2$.

is included in the model, the YSP contributes a slightly higher proportion of the flux than the OSP, and that when a power-law component is included, this difference becomes more pronounced. Fig. A13 shows an example of an acceptable fit using combination 2, including a YSP of $t_{\mathrm{YSP}}=0.02$ and $E(B-V)=0.2$.

\section{A12 J0332-00}

Although this object was originally classified by Zakamska et al. (2003) as a type II quasar, the very broad $\mathrm{H} \alpha$ component detected in the Gemini GMOS-S spectrum presented in Fig. 1 clearly shows that J0332-00 is, in fact, a type I AGN. This finding is confirmed by Barth et al. (2014), who also find clear evidence for a broad $\mathrm{H} \alpha$ component in their data. Despite this, an attempt was made to fit the stellar populations in the same manner as the other objects presented in this section.

In Bessiere et al. (2012), J0332-00 was classified as a double nucleus, with the two components separated by $4 \mathrm{kpc}(\sim 0.9 \operatorname{arcsec})$. In this case, the 1.5 arcsec slit passes directly through both nuclei, allowing the extraction of two distinct spatial apertures centred on each of the components (i.e. the quasar host galaxy and the companion nucleus). Fig. A14 shows the positioning of the spectroscopic slit overlaid on our Gemini image, with the positions of the two extracted apertures highlighted. The figure also shows a cross-section of the 2D spectrum with the position of both apertures highlighted. The centres of the two apertures are separated by $\sim 8.0 \mathrm{kpc}$. Each aperture was modelled independently and discussed individually below.

\section{A12.1 Quasar nucleus}

It is interesting to note that the quasar appears to be relatively unreddened given the slope of the power-law component $(\alpha=-2.24)$ in the combination 1 fit shown in Fig. A15 (top panel). This would imply that, if no YSP component is present, the quasar nucleus has a relatively low luminosity considering the high [O III] luminosity. 

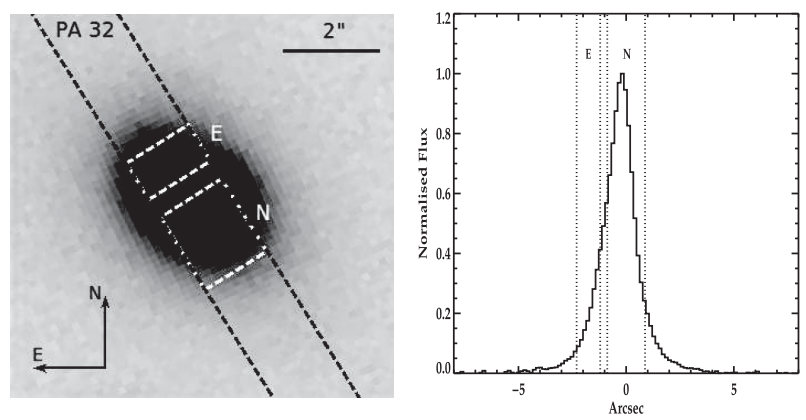

Figure A14. The left-hand panel shows our Gemini GMOS-S image of J0332-00 with the spectroscopic slit overlaid (PA 32). The positions of the two extracted apertures are also shown. The right-hand panel shows a cross-section of the 2D spectrum of J0332-00 showing the position of each extracted apertures. The cross-section was extracted at an observed wavelength of $6185-6215 \AA$ (4720-4745 $\AA$ in the rest frame).
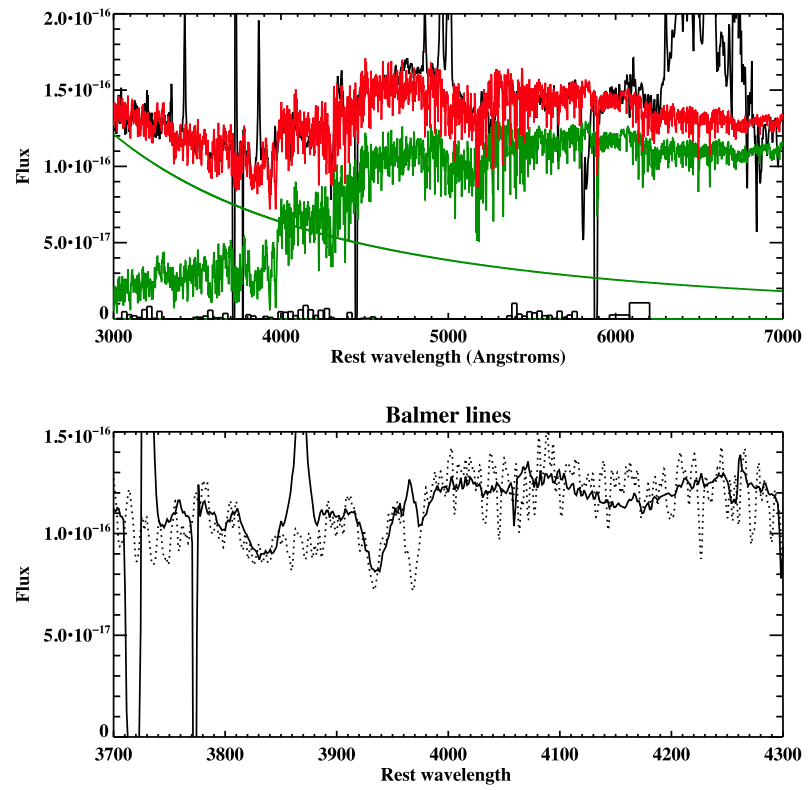

Figure A15. An example of an acceptable fit produced by CONFIT for J0332-00 - quasar host galaxy. The fit shown here was produced using combination 1 and comprises an 8 Gyr population and a power-law component with $\alpha=-2.24$.

In order to determine if this is the case, an attempt to fit the broad $\mathrm{H} \alpha$ and $\mathrm{H} \beta$ components has been made, in order to determine the Balmer decrements in the BLR. This is complicated by the fact that, due to the strong fringing and residuals from the sky subtraction, the spectrum is very noisy at wavelengths greater than $6000 \AA$. This object also has a double peaked broad emission line profile that requires a minimum of two broad components, further complicating the fitting process. We measure the blueshifted component to have $\mathrm{FWHM}=6683 \mathrm{kms}^{-1} \pm 992 \mathrm{kms}^{-1}$ and the redshifted component to have FWHM $=12873 \mathrm{kms}^{-1} \pm 1427 \mathrm{kms}^{-1}$. The Balmer decrements were derived for each of these components separately, and in both cases it was found that $\mathrm{H} \alpha / \mathrm{H} \beta \sim 10$, which is substantially larger than expected for Case B recombination. This is likely due to the poor quality of the data in the region of $\mathrm{H} \alpha$, as well as the uncertainty in the measurement of the $\mathrm{H} \beta$ flux, due to the associated uncertainty in the continuum subtraction. An alternative explanation may be that the BLR reddening is, in fact, large; however, the shorter wavelengths may be dominated by the YSP. Fig. A15
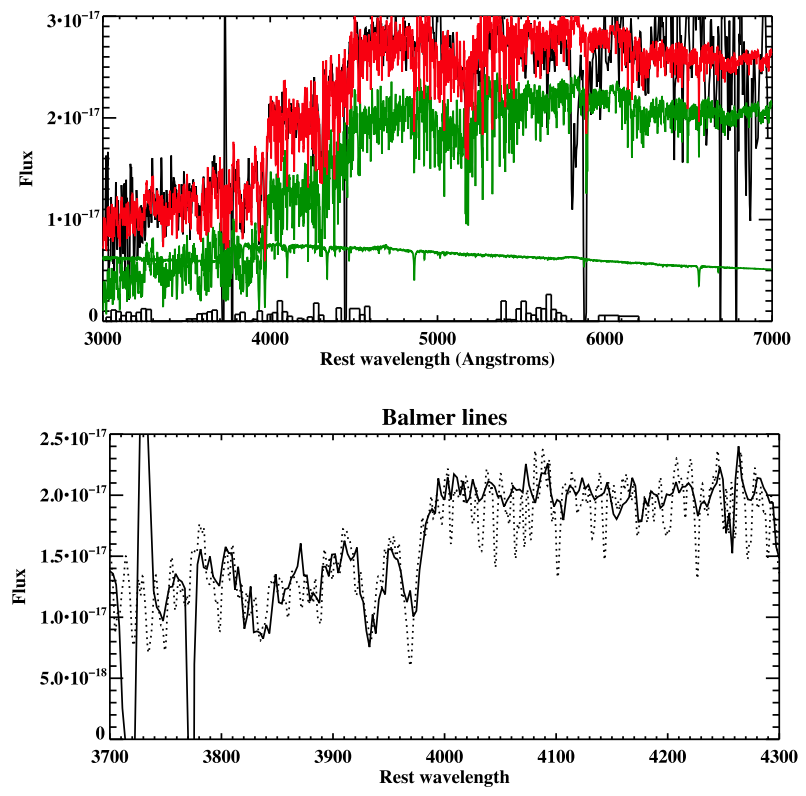

Figure A16. An example of an acceptable fit produced by CONFIT for J0332 - companion nucleus. This particular fit was generated using combination 2 and incorporates a YSP with $t_{\mathrm{YSP}}=0.005 \mathrm{Gyr}$ and $E(B-V)=0.6$.

shows the fit generated by combination 1 , and it can be seen that the combination of an $8 \mathrm{Gyr}$ OSP and a power-law component provides an adequate fit.

\section{A12.2 Companion nucleus}

The initial modelling for the companion was performed using combination 2, and resulted in a best-fitting model that included an unreddened YSP with $t_{\mathrm{YSP}}=0.9 \mathrm{Gyr}$. Measuring the $\mathrm{H} \beta$ flux from the resulting stellar-subtracted spectrum generated a nebular continuum model that contributes $\sim 3$ percent of the flux just below the Balmer edge. This is consistent with the value found for the aperture centred on the quasar nucleus. Once again, it was not possible to obtain a reliable estimate for the Balmer decrements, and therefore, an unreddened nebular continuum was subtracted before proceeding with the stellar synthesis modelling.

Even though the companion does not host an AGN, its proximity to the type I quasar means that the extent to which this aperture will be contaminated by scattered light is unclear, and therefore, all five combination of components were run. Tables 5 and 6 shows that only combinations that include an $8 \mathrm{Gyr}$ component produce acceptable fits. All combinations that include a 2 Gyr ISP do produce solutions with $\chi_{\text {red }}^{2}<1$; however, these invariably overpredict the $\mathrm{Ca}$ II $\mathrm{K}$ absorption feature and were therefore rejected. Fig. A16 shows an example of an acceptable fit produced using combination 2 . In this case, the YSP has age $t_{\mathrm{YSP}}=0.005 \mathrm{Gyr}$ and $E(B-V)=0.6$.

\section{A13 J0334+00}

The initial modelling for J0334+00 was performed using combination 2 , and resulted in a best-fitting model with $t_{\mathrm{YSP}}=0.03 \mathrm{Gyr}$ and $E(B-V)=0.1$. The nebular continuum model then contributes $\sim 22$ percent of the flux in the 3540-3640 $\AA$ region. The Balmer decrements were measured using both the $\mathrm{H} \gamma / \mathrm{H} \beta$ and $\mathrm{H} \delta / \mathrm{H} \beta$ ratios (Table 4). The two measurements result in different ranges of possible reddenings, and therefore, a value of $E(B-V)=0.36$ was 

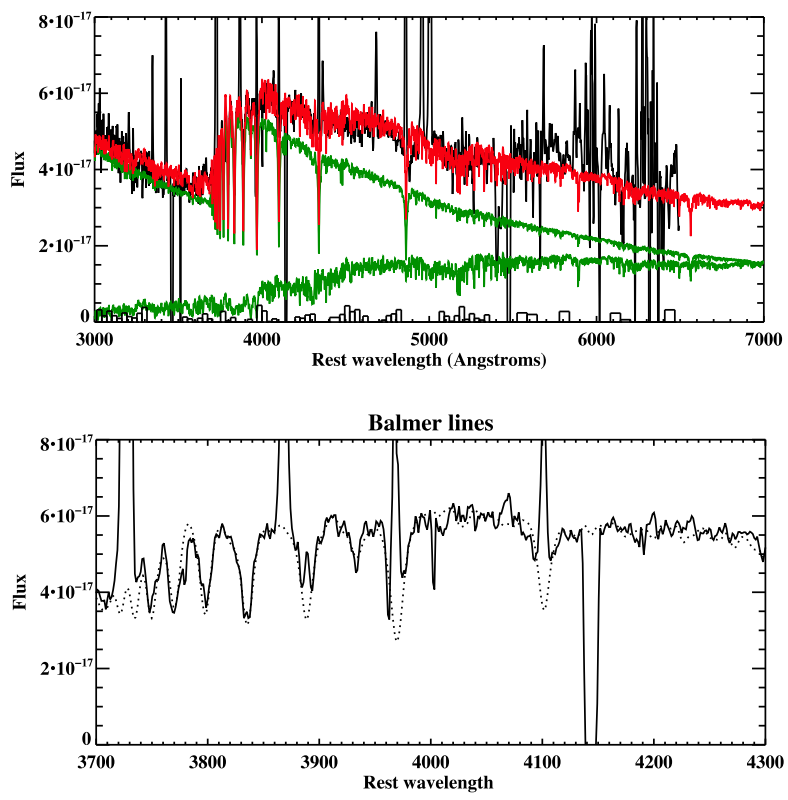

Figure A17. An example of an acceptable fit produced by CONFIT for J0334+00. The fit shown here was produced using combination 2 and includes a YSP with $t_{\mathrm{YSP}}=0.08 \mathrm{Gyr}$ and $E(B-V)=0$.

assumed because this is the average value of the region in which the values overlap. However, this resulted in slightly poorer fits than that was achieved in the initial run, and therefore, an unreddened nebular model was subtracted before proceeding with the modelling.

Tables 5 and 6 show that all four combinations that include a YSP component are successful. When no power-law component is included, the upper age is always less that $100 \mathrm{Myr}$, with low values of reddening for the YSP population $(0 \leq E(B-V) \leq 0.2)$. The inclusion of a power law allows a wider spread of ages and reddenings with a maximum age of $0.4 \mathrm{Gyr}$ and reddening in the range $0 \leq E(B-V) \leq 0.4$. In all cases for which successful fits are achieved, the minimum allowable age is 0.04 Gyr. Fig. A17 shows an example of an acceptable CONFIT fit produced by combination 2 . This particular fit has $t_{\mathrm{YSP}}=0.08 \mathrm{Gyr}$ and $E(B-V)=0$.

\section{A14 J0848+01}

The initial modelling for J0848+01 was carried out using combination 2 , and the resulting best-fitting model comprised a YSP $t_{\mathrm{YSP}}=$ 0.05 with $E(B-V)=0.4$. The nebular continuum generated for this object contributes $\sim 19$ percent of the flux just below the Balmer edge. J0848+01 has intrinsically low equivalent width $\mathrm{H} \gamma$ and $\mathrm{H} \delta$ emission lines, and therefore, it was not possible to make a reliable measurement of the Balmer decrements in order to estimate the reddening, and an unreddened nebular continuum was subtracted.

Tables 5 and 6 show that all the models that included a YSP component produced successful fits with fairly consistent ages of $0.2 \leq t_{\text {YSP }} \leq 0.5$ Gyr. In nearly all the successful fits, the YSP contributes $<50$ per cent of the total flux of the system. Fig. A18 shows an example of an acceptable fit generated by combination 2, which utilizes a YSP $t_{\mathrm{YSP}}=0.2 \mathrm{Gyr}$ and $E(B-V)=0.3$.

\section{A15 J0904-00}

The initial modelling for J0904-00 was carried out using combination 2, which resulted in a best-fitting model with $t_{\mathrm{YSP}}=0.02 \mathrm{Gyr}$ and $E(B-V)=0.3$. After subtracting this model, the flux of $\mathrm{H} \beta$
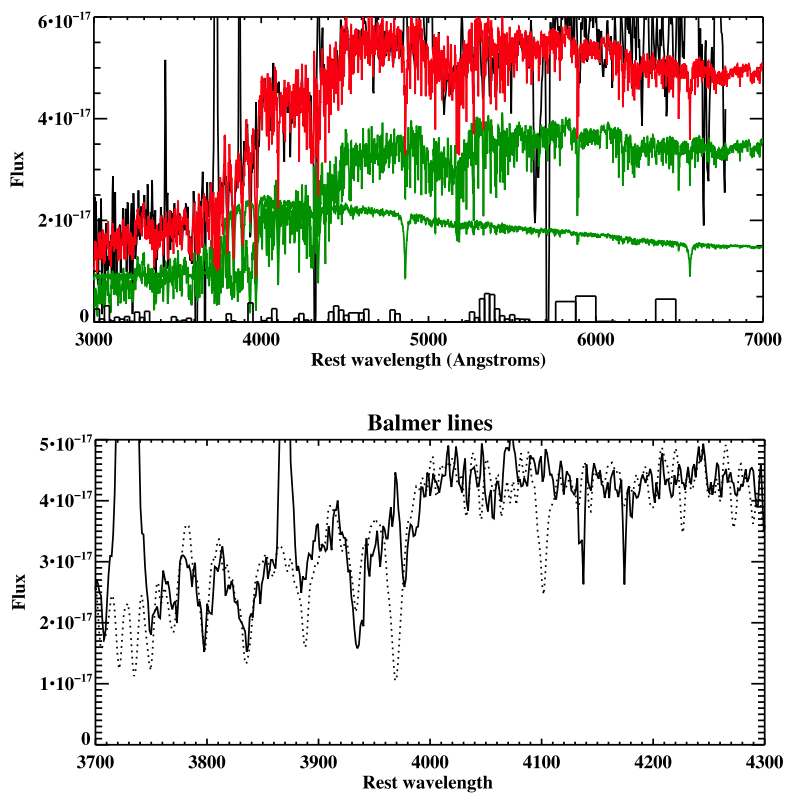

Figure A18. An example of an acceptable fit produced by CONFIT for J0848+01. The fit shown here was generated using combination 2 and include a YSP with $t_{\mathrm{YSP}}=0.2 \mathrm{Gyr}$ and $E(B-V)=0.3$.
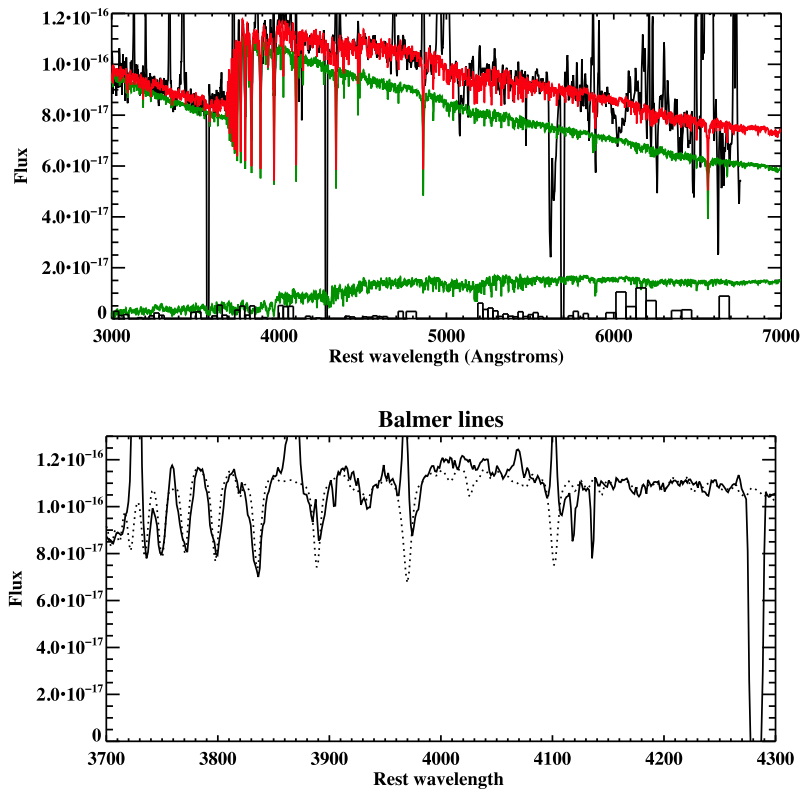

Figure A19. An example of an acceptable fit produced by CONFIT for J0904-00. This fit was produced using combination 2 and includes a YSP with $t_{\mathrm{YSP}}=0.006 \mathrm{Gyr}$ and $E(B-V)=0.4$.

was measured from the resulting spectrum in order to construct the nebular model, which contributes $\sim 13$ per cent to the total flux just below the Balmer edge. No reddening was applied to the nebular continuum before subtraction, because the $\mathrm{H} \gamma / \mathrm{H} \beta$ ratio is consistent with Case B recombination (Table 4), and thus it was deemed unnecessary to do so.

Tables 5 and 6 show the results of the stellar synthesis modelling performed for J0904-00, and shows that all combinations that require two stellar components produce an acceptable fit. However, combination 1 does not produce an adequate result in this case. This is reinforced in Fig. A19 (bottom panel), which demonstrates that 

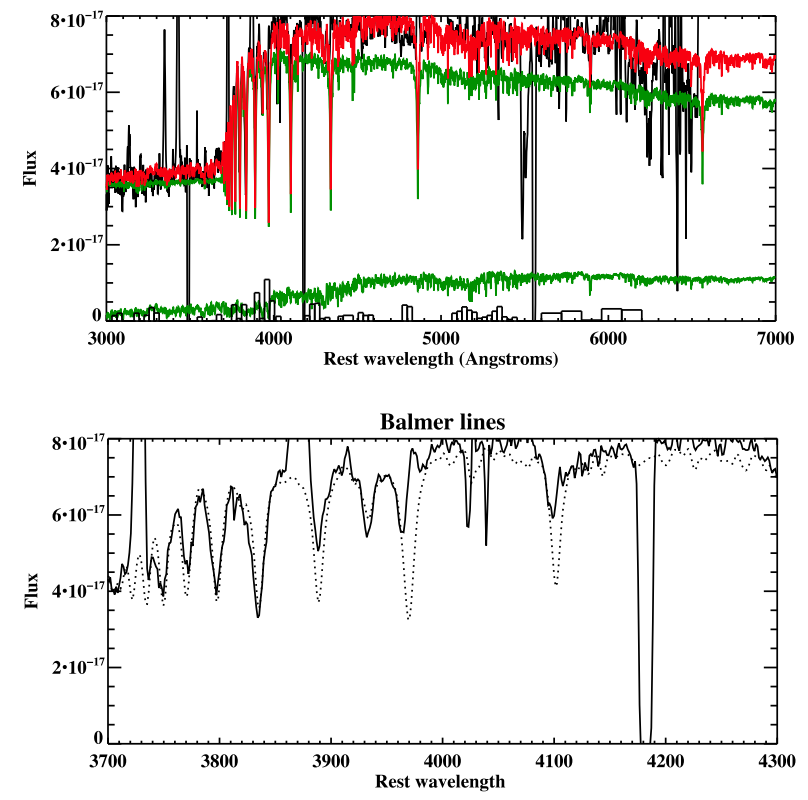

Figure A20. An example of an acceptable fit produced by CONFIT for J0923+01. The fit shown here was generated using combination 2 and incorporates a YSP with $t_{\mathrm{YSP}}=0.05 \mathrm{Gyr}$ and $E(B-V)=0.5$.

the Balmer lines are clearly detectable in the spectrum, meaning that a YSP component is required. Fig. A19 shows an example of an acceptable combination 2 fit for J0904-00, which includes a YSP with $t_{\mathrm{YSP}}=0.006 \mathrm{Gyr}$ and $E(B-V)=0.4$.

\section{A16 J0923+01}

The initial modelling for J0923+01 was performed using combination 2, which resulted in a best-fitting model that included a YSP with $t_{\mathrm{YSP}}=0.04 \mathrm{Gyr}$ and $E(B-V)=0.5$. This model was subtracted from the observed data, and the $\mathrm{H} \beta$ flux was then measured from the stellar-subtracted spectrum, which resulted in a nebular continuum model that contributes $\sim 14$ per cent of the total flux in the 3540-3640 A range. It was not possible to obtain a reliable estimate of the Balmer decrements in this case because the errors in the ratios of $\mathrm{H} \delta / \mathrm{H} \beta$ and $\mathrm{H} \gamma / \mathrm{H} \beta$ were larger than the measured value. Therefore, an unreddened nebular continuum was subtracted from the data, which did not result in an unphysical step in the spectrum at the Balmer edge, and was thus considered acceptable.

Tables 5 and 6 show that, all the combinations that include a YSP component (2-5) produce acceptable fits, all of which allow for a contribution of up to 100 per cent of the flux in the normalizing bin from the YSP. Fig. A20 shows an example of an acceptable fit generated using combination 2. In this case, the fit includes a YSP with age $t_{\mathrm{YSP}}=0.05 \mathrm{Gyr}$ and $E(B-V)=0.5$.

\section{A17 J0924+01}

The initial modelling of J0924+01 was performed using combination 3 , which produced a best-fitting model with $t_{\mathrm{YSP}}=0.003$ and $E(B-V)=1.1$. The resulting nebular continuum model then contributes $\sim 37$ percent of the total flux below the Balmer edge. The Balmer decrements were measured using both the $\mathrm{H} \gamma / \mathrm{H} \beta$ and $\mathrm{H} \alpha / \mathrm{H} \beta$ ratios; however, both values produce large errors. Therefore, the nebular continuum was initially reddened using a value of $E(B-V)=0.7$, which resulted in an under subtraction of the nebular component. In order to test various values of reddening for
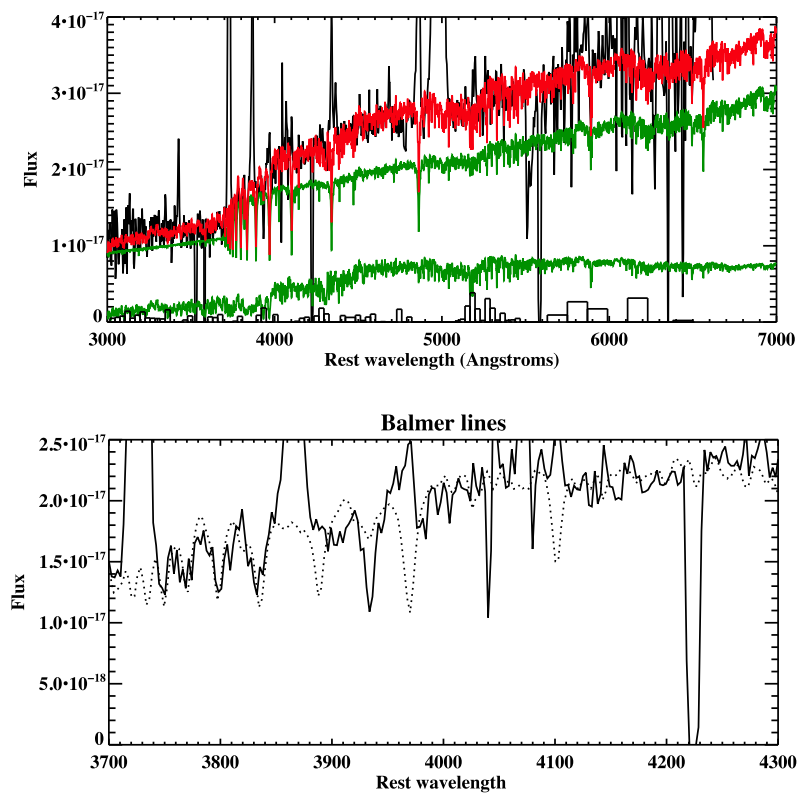

Figure A21. An example of an acceptable fit produced by CONFIT for J0924+01. The combination shown here was produced using combination 2 and includes a YSP with $t_{\mathrm{YSP}}=0.02 \mathrm{Gyr}$ and $E(B-V)=0.8$.

the model nebular continuum, reddening beginning with the lowest value determined from the Balmer decrements $(E(B-V)=0.2)$ was applied, and then increased in increments of $\Delta E(B-V)=0.05$, until the point at which the continuum subtraction no longer resulted in a step in the spectrum at the Balmer edge $(E(B-V)=0.3$, Table 4$)$. The nebular continuum contributes $\sim 14$ percent of the total flux just below the Balmer edge, after the reddening correction has been applied.

J0924+01 was modelled using all five combinations of components outlined in Section 3.2, and Tables 5 and 6 show that all combinations that include a YSP/ISP component produce acceptable fits. When considering combinations 2 and 3, the range of acceptable fits is very limited, with combination 2 producing two solutions with $\chi_{\text {red }}^{2}<1$, in which the YSP dominates the flux, and combination 3 producing only one solution. When using combinations 4 and 5, which both include a power-law component, a wide range of solutions produce $\chi_{\text {red }}^{2}<1$, and in both cases all solutions are acceptable, meaning that these combinations do not provide any real constraint on the age and/or reddening of any YSP present.

Fig. A21 shows an example of an acceptable fit produced by CONFIT, which was generated using combination 2. This fit includes a YSP with $t_{\mathrm{YSP}}=0.02 \mathrm{Gyr}$ and $E(B-V)=0.8$. Fig. A21(b) shows that the strength of the $\mathrm{Ca}$ II $\mathrm{K}$ line is strongly underpredicted by the model, which is consistent with the high value of reddening determined here. This is because, the gas and dust that are responsible for the reddening will enhance this feature. In this case, it is relatively easy to search for $\mathrm{Ca}$ II $\mathrm{K}$ absorption due to the reddening dust column because the YSP dominates the flux; however, when the YSP contributes a lower proportion of the flux, this effect would be more difficult to detect.

\section{A18 J0948+00}

The initial modelling for J0948+00 was performed using combination 3 and resulted in a best-fitting model that included a YSP with $t_{\mathrm{YSP}}=0.1 \mathrm{Gyr}$ and $E(B-V)=0.3$. Having subtracted this model 

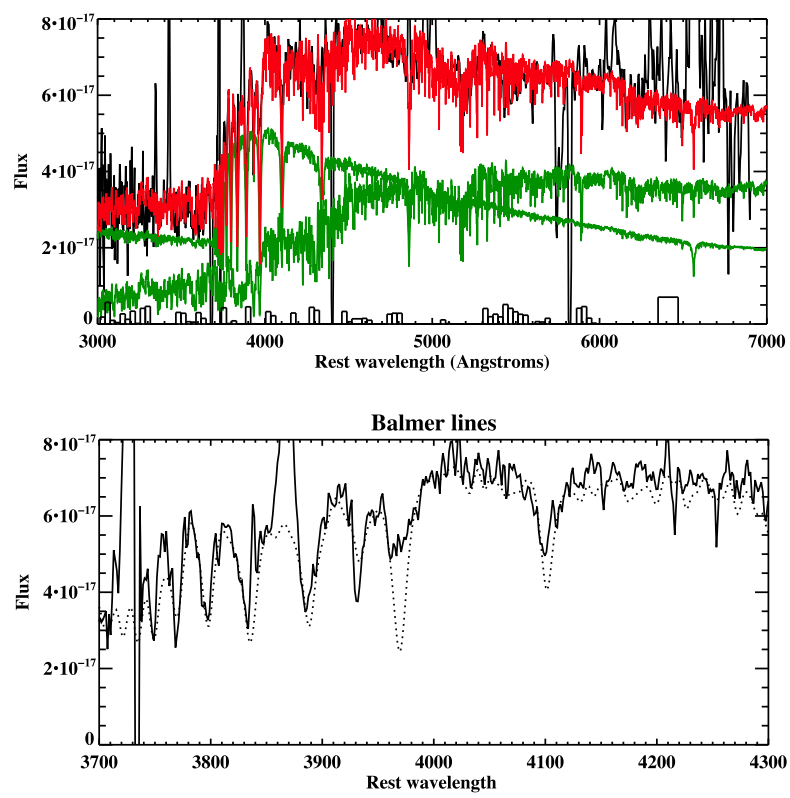

Figure A22. An example of an acceptable fit produced by CONFIT for J0948+00. The fit shown here was generated using combination 2 and includes a YSP with $t_{\mathrm{YSP}}=0.2 \mathrm{Gyr}$ and $E(B-V)=0.1$.

from the spectrum, the nebular continuum model generated from the measured $\mathrm{H} \beta$ flux contributes $\sim 15$ per cent of the total flux below the Balmer edge, and is thus relatively weak. It was not possible to make a reliable measurement of the Balmer decrements because of the low equivalent widths of the $\mathrm{H} \gamma$ and $\mathrm{H} \delta$ lines. Therefore, an unreddened nebular continuum model was subtracted, which did not result in an unphysical step at the Balmer edge.

All five modelling combinations, which are outlined in Section 3.2, were applied to the spectrum and the results, given in Tables 5 and 6, show that all combinations that include a YSP component provide acceptable fits.

On the basis of the deep $r^{\prime}$-band Gemini GMOS-S image (Bessiere et al. 2012), J0948+00 was classified as an undisturbed galaxy; however, the spectrum shows clear evidence of an episode on star formation within the last 0.4 Gyr. This means that like J0142+14 (Section A4), J0948+00 could also be a candidate E+A galaxy. Fig. A22 shows an example of an acceptable fit generated using combination 2, and shows that the OSP and YSP components contribute roughly equal proportions of the total flux in the normalizing bin (4520-4580 $\mathrm{A})$. This fit includes a YSP with $t_{\mathrm{YSP}}=$ 0.2 Gyr and $E(B-V)=0.1$.

\section{A19 J1307-02}

The initial modelling for J1307-01 was carried out using combination 4. Although acceptable fits could be achieved with combination 2 , those which included a power-law component were found to provide better fits around the Balmer absorption features and $G$ band. A stellar model including a YSP $t_{\mathrm{YSP}}=1.8 \mathrm{Gyr}$ and $E(B-V)$ $=0.3$ was subtracted from the data and the nebular continuum generated from the stellar-subtracted spectrum contributes 27 per cent of the total flux just below the Balmer edge. The Balmer decrement was determined from $\mathrm{H} \gamma / \mathrm{H} \beta$ to be $0.40 \pm 0.07$; however, when this reddening correction was applied to the nebular continuum, this resulted in an undersubtraction around the Balmer edge in the data. Therefore, the unreddened nebular continuum was subtracted from
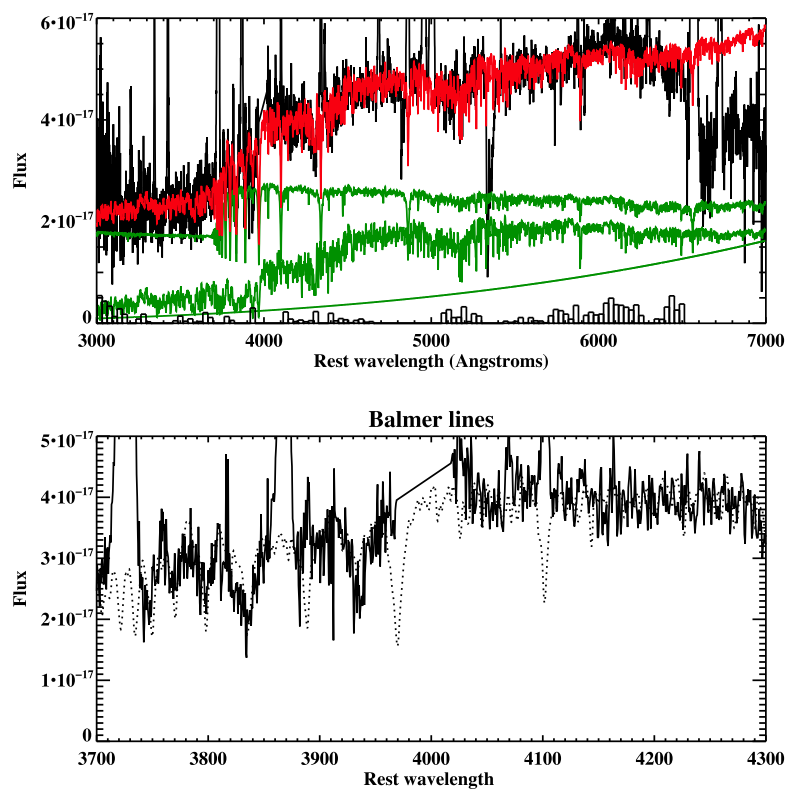

Figure A23. An example of an acceptable fit produced by confIT for J1307-02. The fit shown here was generated using combination 4 and includes a YSP with $t_{\mathrm{YSP}}=0.08 \mathrm{Gyr}$ and $E(B-V)=0.1$.
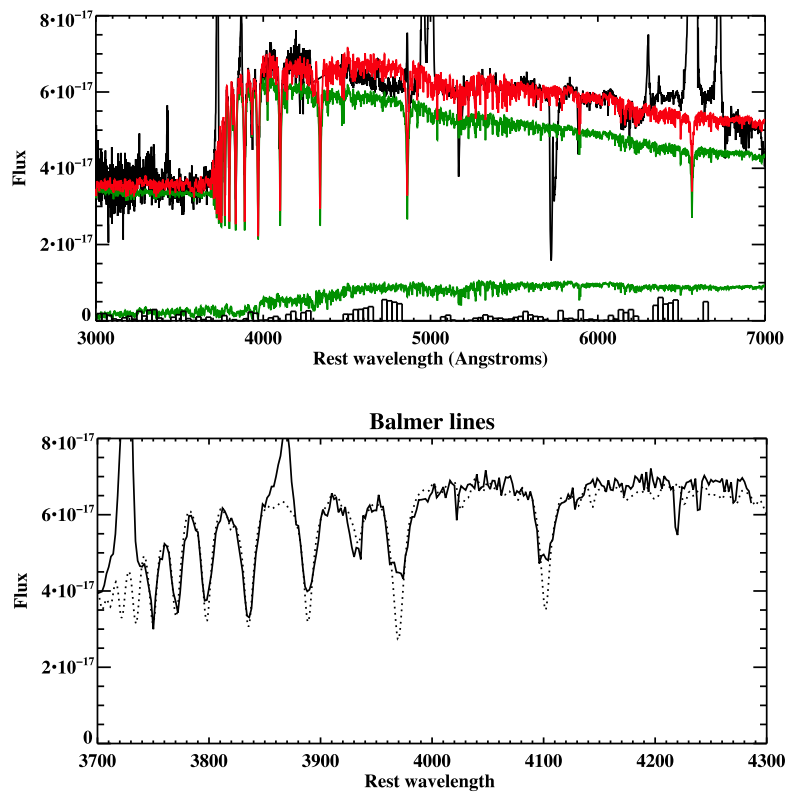

Figure A24. An example of an acceptable fit produced by CONFIT for J1337-01. The fit shown here was generated using combination 2 and includes a YSP with $t_{\mathrm{YSP}}=0.06 \mathrm{Gyr}$ and $E(B-V)=0.4$.

the data before proceeding with the modelling. All five modelling combinations were attempted, the results of which are shown in Tables 5 and 6 . We can see that when an 8 Gyr OSP is assumed, it is only with the inclusion of a power-law component that acceptable fits are achieved. However, in the case where a 2 Gyr underlying population is assumed, acceptable fits can be achieved both with and without a power-law component. Fig. A23 shows an example of an acceptable combination 4 fit for J1307-02, which includes a YSP with $t_{\mathrm{YSP}}=0.08 \mathrm{Gyr}$ and $E(B-V)=0.1$. 


\section{A20 J1337-01}

The initial modelling was carried out for J1337-01 using combination 2 that produced a best-fitting model with a YSP $t_{\mathrm{YSP}}=0.04$ and $E(B-V)=0.5$. This stellar model was subtracted from the data and the $\mathrm{H} \beta$ value flux measured in order to construct the nebular continuum. This nebular model was found to contribute $\sim 8$ per cent of the total flux just below the Balmer edge. The internal reddening was measured using both the $\mathrm{H} \beta / \mathrm{H} \alpha$ and $\mathrm{H} \delta / \mathrm{H} \beta$ ratios and were found to be consistent with Case $\mathrm{B}$, and therefore, no reddening correction was applied to the nebular before subtraction. All the modelling combinations outlined in Section 3.2 were applied, and it can be seen in Tables 5 and 6 that all combinations that include a YSP are successful in producing acceptable results, some of which will allow for a 100 per cent contribution to the flux in the normalizing bin by the YSP. Fig. A24 shows an example of an acceptable fit generated using combination 2 and including a YSP with $t_{\mathrm{YSP}}=$ $0.06 \mathrm{Gyr}$ and $E(B-V)=0.4$.

This paper has been typeset from a $\mathrm{T}_{\mathrm{E}} \mathrm{X} / \mathrm{L} \mathrm{T} \mathrm{E} \mathrm{X}$ file prepared by the author. 